\title{
Ultrafast Infrared Studies of Complex Ligand Rearrangements in Solution
}

\author{
by
}

Christine K. Payne

B.S. (University of Chicago) 1998

A dissertation submitted in partial satisfaction of the requirements for the degree of

Doctor of Philosophy

in

Chemistry

in the

GRADUATE DIVISION

of the

UNIVERSITY OF CALIFORNIA, BERKELEY

Committee in charge:

Professor Charles B. Harris, Chair

Professor Graham R. Fleming

Professor Roger W. Falcone

Spring 2003 
The dissertation of Christine K. Payne is approved:

\begin{tabular}{lr}
\hline Chair & Date \\
\hline & \\
\hline Date \\
\hline
\end{tabular}

University of California, Berkeley

Spring 2003 
Ultrafast Infrared Studies of Complex Ligand Rearrangements in

\section{Solution}

Copyright 2003

by

Christine K. Payne 


\begin{abstract}
Ultrafast Infrared Studies of Complex Ligand Rearrangements in Solution

by

Christine K. Payne

Doctor of Philosophy in Chemistry

University of California, Berkeley

Professor Charles B. Harris, Chair
\end{abstract}

The complete description of a chemical reaction in solution depends upon an understanding of the reactive molecule as well as its interactions with the surrounding solvent molecules. Using ultrafast infrared spectroscopy it is possible to observe both the solutesolvent interactions and the rearrangement steps which determine the overall course of a chemical reaction. The topics addressed in these studies focus on reaction mechanisms which require the rearrangement of complex ligands and the spectroscopic techniques necessary for the determination of these mechanisms.

Ligand rearrangement is studied by considering two different reaction mechanisms for which the rearrangement of a complex ligand constitutes the most important step of the reaction. The first system concerns the rearrangement of a cyclopentadienyl ring as 
the response of an organometallic complex to a loss of electron density. This mechanism, commonly referred to as "ring slip", is frequently cited to explain reaction mechanisms. However, the ring slipped intermediate is too short-lived to be observed using conventional methods. Using a combination of ultrafast infrared spectroscopy and electronic structure calculations it has been shown that the intermediate exists, but does not form an eighteenelectron intermediate as suggested by traditional molecular orbital models.

The second example examines the initial steps of alkyne polymerization. Group 6 $(\mathrm{Cr}, \mathrm{Mo}, \mathrm{W})$ pentacarbonyl species are generated photolytically and used to catalyze the polymerization of unsaturated hydrocarbons through a series of coordination and rearrangement steps. Observing this reaction on the femto- to millisecond timescale indicates that the initial coordination of an alkyne solvent molecule to the metal center results in a stable intermediate that does not rearrange to form the polymer precursor. This suggests that polymerization requires the dissociation of additional carbonyl ligands before rearrangement can occur.

Overall, this research demonstrates the importance of examining reaction dynamics on the ultrafast timescale. In the case of both ring slip and alkyne polymerization, early time dynamics have been invaluable in understanding the exact reaction mechanisms which show important differences from previously accepted models. 
Professor Charles B. Harris

Dissertation Committee Chair 
Dedicated to Virginia B. Bedinger and Irma K. Gilroy. 


\section{Contents}

List of Figures $\quad$ v

List of Tables $\quad$ vii

1 Introduction 1

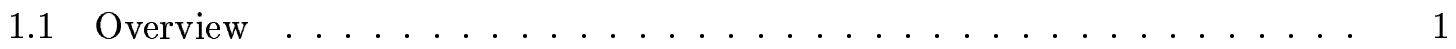

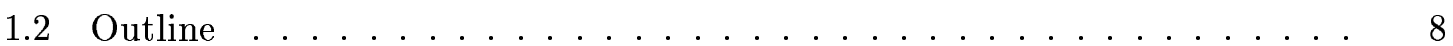

2 Ultrafast Intramolecular Rearrangement: Ring Slip of $\left(\eta^{1}-C_{5} C l_{5}\right) M n(C O)_{5} \mathbf{1 0}$

2.1 Introduction . . . . . . . . . . . . . . . . . 11

2.2 Methods . . . . . . . . . . . . . . . . . . 13

2.2.1 Sample Preparation . . . . . . . . . . . . . . . 13

2.2.2 Femtosecond Infrared Spectroscopy . . . . . . . . . . . . . . . . 14

2.2 .3 Nanosecond Step-Scan FTIR . . . . . . . . . . . . . . . 15

2.2 .4 DFT Calculations . . . . . . . . . . . . . . 15

2.3 Results. . . . . . . . . . . . . . . . . . . 17

2.3.1 Femtosecond Infrared Spectroscopy: $295 \mathrm{~nm}$ Excitation . . . . . . 17

2.3.2 Femtosecond Infrared Spectroscopy: $325 \mathrm{~nm}$ Excitation . . . . . . 20

2.3.3 Nanosecond Step-Scan FTIR . . . . . . . . . . . . . . . 20

2.3.4 DFT Calculations . . . . . . . . . . . . . . 23

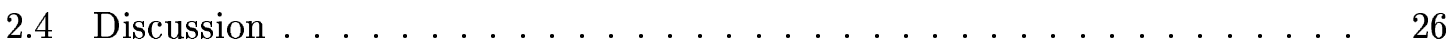

2.4.1 Assignments and Kinetics ............................ 26

2.4 .2 Determination of $\mathbf{D} \ldots \ldots \ldots \ldots \ldots$

2.4.3 Motivation for the Formation of a Seventeen Electron Intermediate . 31

2.4 Mechanism .................... 33

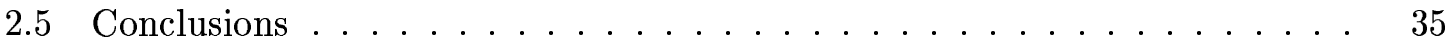


3 Investigation of $\mathrm{M}(\mathrm{CO})_{6}(\mathrm{M}=\mathrm{Cr}, \mathrm{Mo}, \mathrm{W})$ in 1-Hexyne: Understanding the Mechanism of Alkyne Polymerization $\quad 36$

3.1 Introduction . . . . . . . . . . . . . . . . . . . . 36

3.2 Results and Discussion . . . . . . . . . . . . . . . . . 39

3.2.1 Femto- to Picosecond Timescale Spectra . . . . . . . . . . . . 39

3.2.2 Nano- to Millisecond Timescale Spectra . . . . . . . . . . . . . . 41

3.3 Electronic Structure Calculations: Geometry and Frequency . . . . . . . . . 43

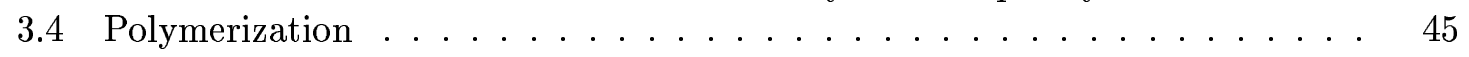

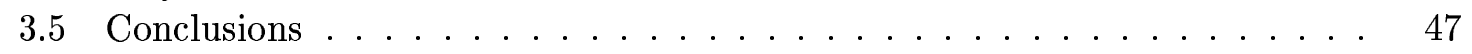

4 Organometallic Solvation: Coordination and Rearrangement of Solvent $\begin{array}{ll}\text { Molecules } & 48\end{array}$

4.1 Introduction . . . . . . . . . . . . . . . . . . 48

4.2 Methods . . . . . . . . . . . . . . . . 55

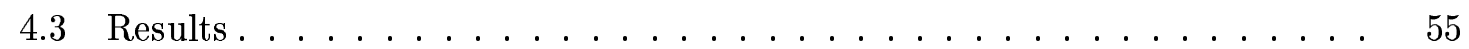

4.3.1 Calculations of Binding Energy . . . . . . . . . . . . . . 57

4.4 Discussion . . . . . . . . . . . . . . . . . . 59

4.4.1 Intermolecular Forces-Dissociation of a Solvent Molecule from the Bulk 62

4.4.2 Dielectric Relaxation-Rearrangement of Solvent Molecules . . . . . 63

4.5 Conclusions . . . . . . . . . . . . . . . . . . 65

5 Methods: An Overview of Experimental and Computational Techniques 66

5.1 Organometallic Photochemistry . . . . . . . . . . . . 66

5.2 Lasers . . . . . . . . . . . . . . . . . . . . 73

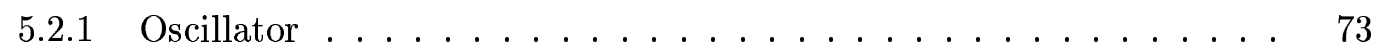

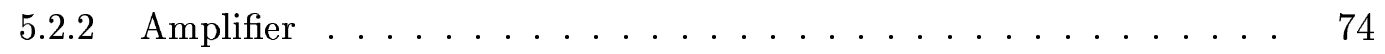

5.3 Nonlinear Crystals . . . . . . . . . . . . . . . . 76

5.4 Time Resolution . . . . . . . . . . . . . . . . 79

5.5 Detection . . . . . . . . . . . . . . . 81

5.6 Data Analysis . . . . . . . . . . . . . . . . . . . . . . . . . 82

5.7 Electronic Structure Calculations . . . . . . . . . . . . . . . 83

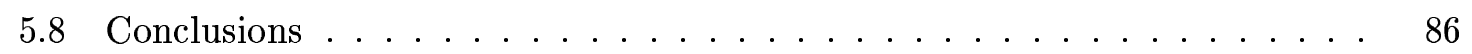

6 Laser I: Ultrafast Ultraviolet-Pump Infrared-Probe Solid State Laser Sys$\begin{array}{ll}\text { tem } & \mathbf{8 7}\end{array}$

6.1 Generation of Ultraviolet, Visible, and Infrared Light . . . . . . . . . . 88

6.1.1 Pump-Ultraviolet and Visible . . . . . . . . . . . . . . . 90

6.1 .2 Probe-Infrared . . . . . . . . . . . . . . . 93

6.2 Overlap of Pump and Probe . . . . . . . . . . . . . 97 
6.3 Detector, Electronics, and Software . . . . . . . . . . . . 98

7 Laser II: Ultrafast Ultraviolet-Pump Infrared-Probe Dye-Amplified Laser $\begin{array}{ll}\text { System } & 100\end{array}$

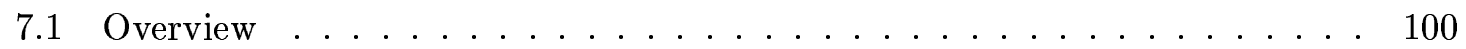

7.2 Experimental Details . . . . . . . . . . . . . . . . 101

8 Step-Scan FTIR: Observation of Dynamics on the Nano- to Microsecond $\begin{array}{ll}\text { Timescale } & 103\end{array}$

8.1 Overview . . . . . . . . . . . . . . . . . . 103

8.2 Detection . . . . . . . . . . . . . . 106

8.3 Experimental Details . . . . . . . . . . . . . . . 108

9 Conclusions $\quad 109$

$\begin{array}{ll}\text { Bibliography } & 111\end{array}$

10 Appendix: Alignment, Maintenance, Comments $\quad 120$

10.1 Alignment and Maintenance of the Ti:Sapphire Oscillator . . . . . . . . . . 121

10.2 Alignment of TOPAS . . . . . . . . . . . . . . . . . . . . . 122

10.3 Maintenance of Additional Laser Components . . . . . . . . . . . . . . . . 125

10.3.1 Verdi Pump Laser for Oscillator . . . . . . . . . . . . . . . 125

10.3.2 Nd:YAG Laser for Pumping the Regenerative Amplifier . . . . . . 125

10.3.3 Regenerative Amplifier . . . . . . . . . . . . . . . 126

10.3.4 Stretcher/Compressor . . . . . . . . . . . . . . . . . . 126

10.3.5 Translation Stage . . . . . . . . . . . . . . . . . . 126

10.3.6 Monochromator . . . . . . . . . . . . . 126

10.3.7 CAMAC/Boxcar Integrators/Computer . . . . . . . . . . . 127 


\section{List of Figures}

2.1 Ultrafast Infrared Spectra of $\left(\eta^{1}-C_{5} C l_{5}\right) M n(C O)_{5}$ in Pentane; $295 \mathrm{~nm}$

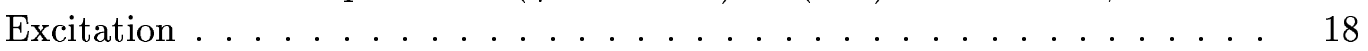

2.2 Ultrafast Kinetics of $\left(\eta^{1}-C_{5} C l_{5}\right) M n(C O)_{5}$ Following Photoexcitation . . . 19

2.3 Ultrafast Infrared Spectra of $\left(\eta^{1}-C_{5} C l_{5}\right) M n(C O)_{5}$ in Pentane; $325 \mathrm{~nm}$

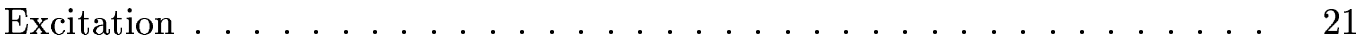

2.4 Nanosecond Kinetics of $\left(\eta^{1}-C_{5} C l_{5}\right) M n(C O)_{5}$ Following Photoexcitation . 22

2.5 DFT Optimized Geometries of Intermediate Species . . . . . . . . . . . 24

2.6 DFT Optimized Geometries of Additional Ring-Slipped Cyclopentadienyl Species . . . . . . . . . . . . . . . . . 27

2.7 Relationship Between Ionization Energy and Ring Slipped Structure . . . . 32

$2.8 \eta^{2}$ and $\eta^{3}$ Bonding Models of Cyclopentadienyl Rings . . . . . . . . . 33

2.9 Mechanism of Ring Slip . . . . . . . . . . . . . . . . . 34

3.1 Proposed Mechanism for the Initiation of Alkyne Polymerization . . . . . 37

3.2 Ultrafast Infrared Spectra of $\mathrm{Mo}(\mathrm{CO})_{6}$ in 1-Hexyne . . . . . . . . . . . . 40

3.3 Millisecond Infrared Spectra of $\mathrm{Mo}(\mathrm{CO})_{6}$ in 1-Hexyne . . . . . . . . . . 42

3.4 DFT Calculated Geometries and Frequencies of Solvated $\mathrm{Mo}(\mathrm{CO})_{5} \ldots \ldots 44$

3.5 FTIR and NMR of Alkyne Polymer . . . . . . . . . . . . 46

4.1 General Photo-Initiated Organometallic Reaction Mechanism . . . . . . . . 49

4.2 Model Site-Specific Potential Energy Surface . . . . . . . . . . . . . . . . . 52

4.3 Bond-line Formulas of Solvents Discussed . . . . . . . . . . . . 56

4.4 Weakly-interacting Solvated Intermediates . . . . . . . . . . . . . 57

4.5 Strongly-interacting Solvated Intermediates . . . . . . . . . . . . . 58

4.6 Long-chain Solvated Intermediates . . . . . . . . . . . . . . 59

4.7 Reaction Rate as a Function of Friction . . . . . . . . . . . . . 61

4.8 Relationship Between Boiling Point and Rearrangement Time . . . . . . . . 62 
4.9 Alcohol Rearrangement Times as a Function of Viscosity and Dielectric Relaxation Rates . . . . . . . . . . . . . . . . 64

5.1 Ultraviolet-Visible Spectra of $\eta^{1}-\left(C_{5} C l_{5}\right) M n(C O)_{5}$ and $M n_{2}(C O)_{10} \ldots 68$

5.2 Metal-Carbonyl Bonding . . . . . . . . . . . . . . . . . 70

5.3 Electron Dynamics of MCT Detectors . . . . . . . . . . . . 81

6.1 Schematic of Solid State Laser System _ . . . . . . . . . . . . . . 89

6.2 Optics Diagram; Ultraviolet Pump Beam . . . . . . . . . . . . . . . . 91

6.3 Optics Diagram; Infrared Probe Beam . . . . . . . . . . . . . . 95

10.1 Optical Diagram of TOPAS . . . . . . . . . . . . . . 124 


\section{List of Tables}

4.1 Solvents and Physical Parameters of Solvent-Solvent Interactions . . . . . 55

6.1 Optics Used for Generation of Ultraviolet Light . . . . . . . . . . . . . . . . . 92

6.2 Description of Infrared Probe Line Components . . . . . . . . . . . . 96 


\section{Acknowledgments}

I would like to thank Charles Harris for creating a wonderful research opportunity. Charles has constantly reminded me to focus on fundamental scientific questions and been generous in allocating time and resources for whatever I felt those questions were. ${ }^{1}$ Additionally, Vijaya Narasimhan deserves much credit for keeping the Harris Group in business.

Working in the Harris Group is an indescribable experience. The existence of both a Surface and Liquid Side allows each group a captive audience of scientific objectivity. From the Surface Side I would especially like to thank Paul Szymanski who has been a great classmate and fellow seeker of real-life examples of quantum mechanics. Chung Wong, Kelly Gaffney, Andre Miller, Simon Liu, Sean Garrett-Roe, Steve Robertson, Matt Strader and Aram Yang have been fantastic as both colleagues and friends.

On the Liquid Side, I have been fortunate to work with two excellent groups of people. Of the first group, Haw Yang, Ken Kotz, and Preston Snee, I would especially like to thank Haw for teaching me a great deal about the relationship between science and hard work. The second group, Libby Garrard, Jennifer Shanoski, and Jason Van Zanten have taught more than they can imagine and been a pleasure to work with. Zack Gainsforth and Cassidy Houchins have helped a great deal in getting difficult projects started. A visit from Matthias Kling made for an enjoyable summer of soccer watching and laser aligning.

I have been very lucky to work with some fantastic collaborators. Laurel Schafer

\footnotetext{
${ }^{1}$ With the notable exception of two-photon microscopy for which I am still hopeful.
} 
and Ben Mork from the Tilley Group have been generous with synthetic assistance and advice. Heinz Frei has helped to teach me about the work and and details involved in research.

Outside of lab, I would like to thank the Payne/Bedinger/Gilroy/Richman/Shaw family for their support over the years. I appreciate both their surprise packages and interest in my research. Ellen and Ken deserve special thanks for adventures outside of lab.

This work was supported by the National Science Foundation and the Director, Office of Science, Office of Basic Energy Science, Chemical Sciences Division, of the U.S. Department of Energy under Contract No. DE-AC03-76SF00098. 


\section{Chapter 1}

\section{Introduction}

\section{$1.1 \quad$ Overview}

The development of organometallic chemistry is reflected in the awarding of the 1963 and 1973 Nobel Prizes in Chemistry. The award in 1963 to Giulio Natta and Karl Ziegler honored work done in the 1950's developing methods for the polymerization of olefins. Their use of a mixed metal catalyst, $\mathrm{TiCl}_{4} / \mathrm{Al}\left(\mathrm{C}_{2} \mathrm{H}_{5}\right)_{3}$, revolutionized the industrial production of plastics[1, 2]. The 1973 award to Ernst Fischer and Geoffrey Wilkinson celebrated the characterization and development of the organometallic "sandwich" compounds $[3,4]$. The best-known of these is ferrocene, consisting of two cyclopentadienyl rings bound to a single iron atom. These compounds represented a new paradigm in chemical bonding in which a single metal atom is bound to a ligand as a whole rather than to 
a specific atom of that ligand. These two prizes demonstrate the breadth of organometallic chemistry, including topics such as catalysis, synthesis, and characterization.

As the field of organometallic chemistry has grown it has become clear that the interactions between central metal atoms and organic ligands result in a great deal of important applications. The two largest groups of applications are centered around catalysis and synthesis. In terms of catalysis the impact of organometallic chemistry has been unmatched. While the use of Ziegler-Natta catalysts for the production of plastics is a very visible example, organometallic catalysts are also used in the production of a wide range of familiar compounds including Vitamin A and ibuprofen[5]. Organometallic compounds have also found applications in a variety of synthetic methods including the synthesis of organic and solid state materials[6]. The use of organometallic compounds allows synthetic chemists a greater degree of control in their synthetic routes.

The wide range of uses for organometallic compounds depends upon the variety of reaction mechanisms. A few examples of reaction mechanisms include bond activation, ligand rearrangement, ligand substitution, and isomerization. Bond activation, the chemically-induced breaking of specific bonds, plays a large role in catalysis. For example, the strong carbon-hydrogen bonds of alkanes make them inert under standard conditions. Only under extreme temperatures, $>1000 \mathrm{~K}$, is it possible to break a carbon-hydrogen bond. Using certain organometallic compounds the activation barrier is substantially lowered making it possible to activate a $\mathrm{C}-\mathrm{H}$ bond under ambient conditions[7, 8, 9]. Ligand 
rearrangements such as $\beta$-hydrogen elimination and ring slip are two examples of unique rearrangement mechanisms. The first, $\beta$-hydrogen elimination, is a major decomposition pathway for organometallic compounds as the hydrogen on a $\beta$-carbon easily dissociates to form a hydride and subsequently the corresponding alkene gas. Ring slip describes the change in coordination of a ligand on a metal center[10]. Ligand substitution and isomerization are familiar mechanisms and are often steps in larger reaction schemes.

Many organometallic reactions, including those described above, have been investigated mechanistically using isotopic labeling, kinetic measurements, and reactivity comparisons $[9,11,12]$. These experiments have provided a foundation for the study of reaction mechanisms, but are either indirect measurements of mechanism or lack the timeresolution necessary to understand the individual steps of the reaction. To overcome these problems, reaction mechanisms have been studied at low $(<100 \mathrm{~K})$ temperatures to decrease the reaction rate to a more easily observable timescale.

While a large number of low-temperature and gas phase studies have been carried out, it is difficult to extend these results to the solution phase, in which these reactions generally occur. ${ }^{1}$ During the course of the reaction the interactions between the solute and the solvent must be considered in order to obtain a complete understanding of the reaction mechanism. The interaction between solute and solvent affects the reaction mechanism both indirectly and directly. Indirect influences include the transfer of energy from the

\footnotetext{
${ }^{1}$ For good examples of low-temperature matrix studies, refer to M.S. Wrighton. For gas phase studies, E. Weitz.
} 
vibrationally excited solute to the surrounding solvent $[13,14]$. Direct influences include geminate recombination in which the solvent serves as a cage. This results in the fast recombination of ligands to the metal center following dissociation[14]. A second direct influence of the solvent is solvation, the formation of a weakly bound ligand between an unsaturated metal center and a solvent molecule[15, 16, 17, 18].

The timescales of the reaction mechanism and solvent interactions dictate the time resolution necessary for the study of such processes. Similarly, the organometallic compound studied determines the wavelengths needed to observe the reaction mechanism[14]. Considering that the timescales for the processes described above are generally on the femto- to picosecond timescale it is necessary to use ultrafast spectroscopy to investigate the reaction mechanisms. In terms of wavelength, it is preferable to initiate the reaction via the photolytic dissociation of a single ligand in order to mimic standard reaction conditions. In the case of carbonyl ligands, this requires, for reasons to be discussed later, ultraviolet light. To follow the resulting dynamics, infrared light provides two advantages. First, changes in peak intensities as a function of time can be used to determine rates of formation and decay for intermediates and products. Second, the infrared region of the spectrum allows for the characterization of intermediates based on frequencies of vibrational modes. This is in contrast to the visible region of the spectrum in which broad, electronic absorptions make the characterization of species difficult.

Overall, these requirements suggest an ultrafast ultraviolet-pump infrared-probe 
experiment in which the reaction is initiated by the ultraviolet dissociation of a ligand and the resulting kinetic and structural changes are observed in the infrared with femtosecond resolution. In many cases, the intermediates observed during the course of these reactions exist only on a femto- or picosecond timescale. While infrared absorption provides some structural information, it is usually necessary to confirm the identification of intermediate species with either carefully chosen synthetic models or electronic structure calculations. Both methods are capable of correlating a structure with infrared frequencies that can then be compared to the infrared frequencies of the short-lived intermediate.

Of particular interest are the reaction mechanisms of large-scale ligand rearrangements. These reactions are difficult to characterize using conventional techniques due to the variety of possible intermediate states and short lifetimes. Examples of such reactions include the rearrangement of cyclopentadienyl, commonly referred to as "ring slip" and the rearrangement of alkynes in the course of polymerization. These reactions represent two classes of ligand rearrangements. The first, ring slip, is an intramolecular rearrangement of an aromatic ligand. The second, alkyne polymerization, is a two step reaction in which coordination to a solvent molecule precedes the rearrangement of the ligand.

To obtain a detailed understanding of ring slip we have carried out an infrared spectroscopic study that examines the photochemistry of $\left(\eta^{1}-C_{5} C l_{5}\right) M n(C O)_{5}$ on a femtosecond to microsecond timescale. Experimental observations reveal the fast formation of the two carbonyl-loss product, $\left(\eta^{5}-C_{5} C l_{5}\right) M n(C O)_{3}$, as well as a two-step mechanism for 
the formation of the ring slipped intermediate, $\left(\eta^{2}-C_{5} C l_{5}\right) M n(C O)_{4}$. Density functional calculations have been used to propose possible structures for the intermediate species. These calculations suggest that the intermediate structure, conventionally described as an $\eta^{3}$ species, is instead best described as a seventeen electron $\eta^{2}$ species. Additional calculations show that the formation of a seventeen electron intermediate may be the general mechanism of many reactions which are characterized as ring slip, a deviation from the standard eighteen-electron rule of organometallic chemistry[19].

The polymerization of alkynes is catalyzed by a variety of organometallic complexes. The most simple system consists of the catalysis of terminal alkynes by Group 6 $(\mathrm{M}=\mathrm{Cr}, \mathrm{Mo}, \mathrm{W})$ hexacarbonyl species, $\mathrm{M}(\mathrm{CO})_{6}$. This process had been thought to occur via the photodissociation of a single carbonyl followed by the coordination and rearrangement of a neighboring alkyne solvent molecule. It was suggested that the alkyne solvent molecule would rearrange via either a 1, 2- hydrogen migration or an oxidative addition and 1, 3-shift of the hydride to form the corresponding vinylidene species[20,21, 22]. Dissociation of a second carbonyl and the repetition of the same coordination and rearrangement steps was thought to lead to the eventual polymerization of the alkyne. Ultrafast spectroscopic studies confirm the photodissociation of a carbonyl followed by the coordination of an alkyne molecule. However, no rearrangement to the vinylidene intermediate is observed on the femto- to millisecond timescale. These results suggest that the rearrangement is a multi-photon event, most likely following the dissociation of a second carbonyl[23]. 
The interaction of the Group 6 hexacarbonyls with alkyne solvent molecules provides an interesting point of comparison to previous studies of Group 6 hexacarbonyls in alcohol and silane solutions[15]. These studies had demonstrated that the photodissociation of a carbonyl would be followed by the fast solvation by a neighboring solvent molecule. This is the same mechanism observed in the alkyne studies. Both alcohols and silanes were shown to bind to the metal in one of two confirmations. One possibility was for the metal to bind to the alkyl chain. This would form a weak bond that would quickly dissociate to form a more strongly bound complex with the hydroxyl or silyl group. If the initial coordination of the solvent molecule occurred at the hydroxyl or silyl group no further rearrangement was observed. However, the binding energies of both the alkyl chain and triple bond of the alkyne species are comparable to the corresponding sites in alcohols and no coordination to the alkyl chain is observed for alkynes. Instead solvation only occurs via the triple bond. A comparison between these solvents provides an ideal system to consider how both bulk and microscopic solvent properties influence a reaction.

It is hoped that these studies will provide some insight into the role of complex ligand dynamics during the course of a chemical reaction in solution. 


\subsection{Outline}

Overall, this thesis will describe work aimed at understanding the reaction mechanism of large-scale ligand rearrangements such as the rearrangement of cyclopentadienyl and alkyne ligands during the course of a reaction. Also discussed is the role that the solvent plays in the initial solvation step of such reaction mechanisms. Additionally, the laser system and electronic structure calculations necessary to determine reaction mechanisms are described.

The structure of the thesis is described below. The first two chapters examine the reaction mechanisms of ring slip and alkyne polymerization, respectively. The third chapter describes the binding of a neighboring solvent molecule to a coordinatively unsaturated organometallic complex. This is the first step of alkyne polymerization as well as a host of other reaction mechanism. Of particular interest is the importance of binding energy in determining the rate of the reaction. The fourth chapter provides an overview of the methods used to understand these reaction mechanisms. It is hoped that this chapter will provide a connection between the techniques used and the physical processes occurring in a way that will be helpful for students as they start similar research projects. The fifth, sixth, and seventh chapters provide more specifics of the laser systems used in these experiments. The first of these sections details the generation of the necessary ultraviolet and infrared light as well as the electronics and software for data collection. The second summarizes 
the dye-amplified laser system used for the collection of data, and third describes the stepscan FTIR system used to obtain nano- to millisecond data. The appendix provides all laboratory-specific details such as alignment procedures and fixes to common problems. 


\section{Chapter 2}

\section{Ultrafast Intramolecular}

\section{Rearrangement: Ring Slip of$$
\left(\eta^{1}-C_{5} C l_{5}\right) M n(C O)_{5}
$$

In order to obtain a detailed understanding of the organometallic mechanism known as "ring slip" we have carried out an infrared spectroscopic study that examines the photochemistry of $\left(\eta^{1}-C_{5} C l_{5}\right) M n(C O)_{5}$ on a femtosecond to microsecond timescale. Experimental observations reveal the fast formation of the two carbonyl-loss product, $\left(\eta^{5}-\right.$ $\left.C_{5} C l_{5}\right) M n(C O)_{3}$, as well as a two-step mechanism for the formation of the ring slipped intermediate, $\left(\eta^{2}-C_{5} C l_{5}\right) M n(C O)_{4}$. Density functional calculations have been used to 
propose possible structures for the intermediate species. These calculations suggest that the intermediate structure, conventionally described as an $\eta^{3}$ species, is instead best described as a seventeen electron $\eta^{2}$ species. Additional calculations show that the formation of a seventeen electron intermediate may be the general mechanism of many reactions which are characterized as ring slip, a deviation from the standard eighteen-electron rule of organometallic chemistry. This deviation is found to be dependent on the ionization energy of the metal center.

\subsection{Introduction}

Ring slip is a commonly proposed, yet poorly understood, mechanism of organometallic chemistry used to describe the change in hapticity of a ligand on a transition metal center $[10,24,25]$. Reactions for which it is proposed take advantage of the decrease in hapticity, or slip of the ring, to maintain an eighteen electron count on the metal center throughout the reaction. Mechanisms involving ring slip have been proposed for a variety of organometallic reactions including substitutions[26, 27, 28], carbon-hydrogen[29], siliconhydrogen[30] and carbon-carbon[31] activation, as well as $\alpha$ - and $\beta$-hydrogen transfer[32].

The first reaction for which ring slip was proposed was the substitution of a twoelectron ligand in the $\mathrm{CpRh}(\mathrm{CO})_{2}\left[\mathrm{Cp}=\mathrm{C}_{5} \mathrm{H}_{5}\right]$ compound[33]. The formulation of the ring slip mechanism was based on kinetic studies which suggested an associative substitution. 
To avoid postulating an unfavorable twenty electron compound it was suggested that the cyclopentadienyl $(\mathrm{Cp})$ ring rearranged from $\eta^{5}$ to $\eta^{3}$ as a two-electron ligand was substituted at the rhodium center. This preserved the eighteen electron count of the metal throughout the reaction. Despite the prevalence of $\mathrm{Cp}$ rings in organometallic chemistry, few discussions of ring slip have focused on the dynamics of the ring slip of the Cp ring. Instead studies have focused on species for which crystal structures are more easily obtained.[24, 25, 34, 35] To the best of our knowledge, there exists only one crystal structure showing a Cp ring in the reduced hapticity, $\eta^{3}$, form. This single example is the crystal structure of $\mathrm{Cp}_{2} \mathrm{~W}(\mathrm{CO})_{2}$ which shows one of the Cp rings in a bent configuration[36]. While this provides an important confirmation of the existence of a $\mathrm{Cp}$ ring in the reduced hapticity form, it does not provide any information regarding the dynamics of the mechanism.

The most notable mechanistic study of a Cp ring undergoing ring slip is that of Young and Wrighton[37]. Their low temperature experiments characterized the "reverse" ring slip of $\left(\eta^{1}-C_{5} C l_{5}\right) M n(C O)_{5}$ as the $\eta^{1}$ species rearranged, following photodissociation, to form both the ring slipped intermediate and the stable $\left(\eta^{5}-C_{5} C l_{5}\right) M n(C O)_{3}$. These experiments formed an important basis for the observation of a species that was an intermediate between the $\eta^{1}$ and $\eta^{5}$ species. Due to the extreme reactivity of the intermediate, they were unable to explore the room temperature dynamics that characterize the system. It is through an understanding of the initial events following photodissociation that the complete mechanism can be understood. For this reason, a time resolved study of ring slip 
in room temperature solution will prove valuable in the determination of the full mechanism of ring slip.

To obtain a dynamic description of ring slip we have expanded the earlier studies of Young and Wrighton through the investigation of the rearrangement of $\left(\eta^{1}-C_{5} C l_{5}\right) M n(C O)_{5}$ on a timescale ranging from femtoseconds to microseconds in room temperature alkane solutions. The interpretation of experimental observations was augmented by density functional theoretical (DFT) calculations. The combination of experimental and theoretical results allows the formulation of the first complete mechanistic description of ring slip for a $\mathrm{Cp}$ ring under ambient conditions. The reverse ring slip reaction will provide information that is relevant to the forward, decreased hapticity, reaction mechanism with regards to the exact nature of the ring slipped intermediate.

\subsection{Methods}

\subsubsection{Sample Preparation}

The $\left(\eta^{1}-C_{5} C l_{5}\right) M n(C O)_{5}$ species was synthesized according to the procedure of Reimer $[38,39]$. Samples for spectroscopic study were prepared using pentane or undecane purchased from Aldrich and purged with nitrogen. Care was taken throughout experiments to maintain a dry nitrogen environment for the solution. Concentration was set for an absorbance of 1 O.D. at the wavelength being pumped, approximately $4 \mathrm{mmol}$ at $295 \mathrm{~nm}$ 
and $6 \mathrm{mmol}$ at $325 \mathrm{~nm}$ with a cell thickness of $250 \mu \mathrm{m}$.

\subsubsection{Femtosecond Infrared Spectroscopy}

Details of the femtosecond IR (fs-IR) spectrometer setup have been published elsewhere.[14] Briefly, the output of a Ti:sapphire oscillator was amplified in series of dye amplifiers pumped by a $30-\mathrm{Hz}$ Nd:YAG laser at $532 \mathrm{~nm}$. The UV and IR beams used to pump and probe the reaction were generated through nonlinear crystals. The specific wavelength of UV light was determined by the choice of dye in the amplification system. The resulting $295 \mathrm{~nm}$ or $325 \mathrm{~nm}$ UV photons, with energy of $6 \mu \mathrm{J} /$ pulse, were focused into a disk of $200 \mu \mathrm{m}$ diameter at the sample to initiate chemical reactions. The $1 \mu \mathrm{J}$ IR pulses were centered at $5 \mu \mathrm{m}$ with a temporal full-width-half-maximum of about $70 \mathrm{fs}$ and a spectral band width of about $200 \mathrm{~cm}^{-1}$. Shot-to-shot fluctuation of the IR probe pulses were minimized by normalizing the signal beam against a reference beam. These two beams were then focused into an astigmatism-corrected spectrographic monochromator (SpectraPro150, Acton Research Corp., $150 \mathrm{gv} / \mathrm{mm}, 4.0 \mu \mathrm{m}$ blazed, to form two spectrally resolved images on a focal-plane-array (FPA) IR detector. The two frequency-resolved images were digitized by two windows of $12 \times 200$ pixels, which allowed simultaneous normalization of a $70 \mathrm{~cm}^{-1}$ spectrum. The censoring chip of the detector was an engineer grade, $256 \times 256$ element $\mathrm{HgCdTe}(\mathrm{MCT})$ matrix of dimensions $1.28 \mathrm{x} 1.28 \mathrm{~cm}^{2}$ (or $50 \mathrm{x} 50 \mu \mathrm{m}^{2}$ per pixel). During the course of an experiment, the censoring chip and its immediate circuits were kept 
in contact with a 4-L liquid nitrogen dewar to increase its sensitivity in the IR range. The typical spectral and temporal resolution for this setup were $4 \mathrm{~cm}^{-1}$ and $300 \mathrm{fs}$, respectively. The polarizations of the pump and the probe pulse were set at the magic angle $\left(54.7^{\circ}\right)$ to ensure that all signals were due to population dynamics.

\subsubsection{Nanosecond Step-Scan FTIR}

The spectra recorded on the nanosecond to microsecond timescale were recorded using a Step-Scan FTIR spectrometer described elsewhere[40]. The instrument was based on a Bruker IFS-88 FTIR with a special scanner module to allow step-scanning. An MCT detector with 37 ns temporal full-width-half-maximum (FWHM) measured from the IR scatter of $1064 \mathrm{~nm}$ light from a YAG laser was used. The IR light was focused in the cavity with two $10 \mathrm{~mm}$ focal length $\mathrm{BaF}_{2}$ lenses, which gave beam sizes smaller than comparable curved mirrors, allowing increased IR throughput and less sample degradation. The sample was photo-excited with $10 \mathrm{~ns}$ pulses from the quadrupled YAG.

\subsubsection{DFT Calculations}

Geometry optimizations and frequency calculations were carried out using the commercial package, JAGUAR, for density functional theory (DFT) calculations[41]. The exchange-correlation functional employed was the Becke's three-parameter hybrid functional[42] combined with the Lee-Yang-Parr (LYP) correlation functional[43], commonly denoted as 
B3LYP[44]. This functional has been shown to give very good results for transition metal complexes[45, 46]. The basis set, denoted LACVP**, was used for all calculations. This basis set consists of the $6-31 \mathrm{G}^{* *}$ basis functions for $\mathrm{H}, \mathrm{C}, \mathrm{O}$, and $\mathrm{Cl}$ atoms $[47,48]$ and the Los Alamos Effective Core Potential (ECP) for Mn and W with the outer-most core orbitals included in the valence description[49].

With the exception of $\left(\eta^{1}-C_{5} C l_{5}\right) M n(C O)_{5}$ and $\left(\eta^{5}-C_{5} C l_{5}\right) M n(C O)_{3}$, molecules with crystal structures that confirmed their symmetry, no constraints were imposed during geometry optimization. To make certain that a proper energy minimum had been located, each geometry optimization was followed by a frequency calculation at the same level of theory. The frequency calculations are reported without scaling factors and are related to experiments based on relative peak positions. Further details of the methods used for generating structures accompany the description of the optimized geometries in order to clarify the results presented.

Solvation calculations were performed using the cyclohexane model solvent provided with Jaguar 3.5. Calculations use a self-consistent reaction field method which begins with a gas phase wavefunction which produces an electrostatic potential. This potential is fit to a set of atomic charges and the reaction field is determined by the numerical solution of the Poisson-Boltzmann equations. The solvent, described by its dielectric constant and probe radius, is represented by a layer of charges at the molecular surface. These charges are then utilized for subsequent wavefunction calculations continuing until the calculations 
are self-consistent $[50,51]$.

\section{$2.3 \quad$ Results}

Spectra are presented in the form of difference spectra in which the loss of the initial species appears as a negative absorption or bleach. ${ }^{1}$

\subsubsection{Femtosecond Infrared Spectroscopy: $295 \mathrm{~nm}$ Excitation}

Spectra taken over a time period of 10 ps to 660 ps are shown in Figure 2.1.

Assignments are based on a combination of previous assignments and kinetic analysis. Both will be discussed in detail below. The bleach of $\left(\eta^{1}-C_{5} C l_{5}\right) M n(C O)_{5}, \mathbf{A}$, can be seen immediately following $295 \mathrm{~nm}$ excitation at $2010 \mathrm{~cm}^{-1}, 2041 \mathrm{~cm}^{-1}, 2076 \mathrm{~cm}^{-1}$ (weak), and $2126 \mathrm{~cm}^{-1}$ although a shorter range of spectra are shown in Figure 1 to highlight the more congested region of the spectrum. Photoproducts are seen at $1980 \mathrm{~cm}^{-1}, 1990 \mathrm{~cm}^{-1}, 2020$ $\mathrm{cm}^{-1} 1,2024 \mathrm{~cm}^{-1}, 2046 \mathrm{~cm}^{-1}$, and $2092 \mathrm{~cm}^{-1}$. With the exception of the peak at 2024 $\mathrm{cm}^{-1}, \mathbf{E}$, and the parent bleach, $\mathbf{A}$, all photoproducts show a fast formation of less than $50 \mathrm{ps}$ and then remain constant over the timescale of observation. The kinetics of $\mathbf{E}$ show a fast rise followed by a long time decay of $252 \mathrm{ps} \pm 5 \mathrm{ps}$. This long time decay correlates with the long time recovery of $\mathbf{A}, 225 \mathrm{ps} \pm 55 \mathrm{ps}$ at $2010 \mathrm{~cm}^{-1}$, shown in Figure 2.2.

\footnotetext{
${ }^{1} \mathrm{~A}$ comparison between the spectra of solutions of pentane and undecane revealed no observable differences.
} 


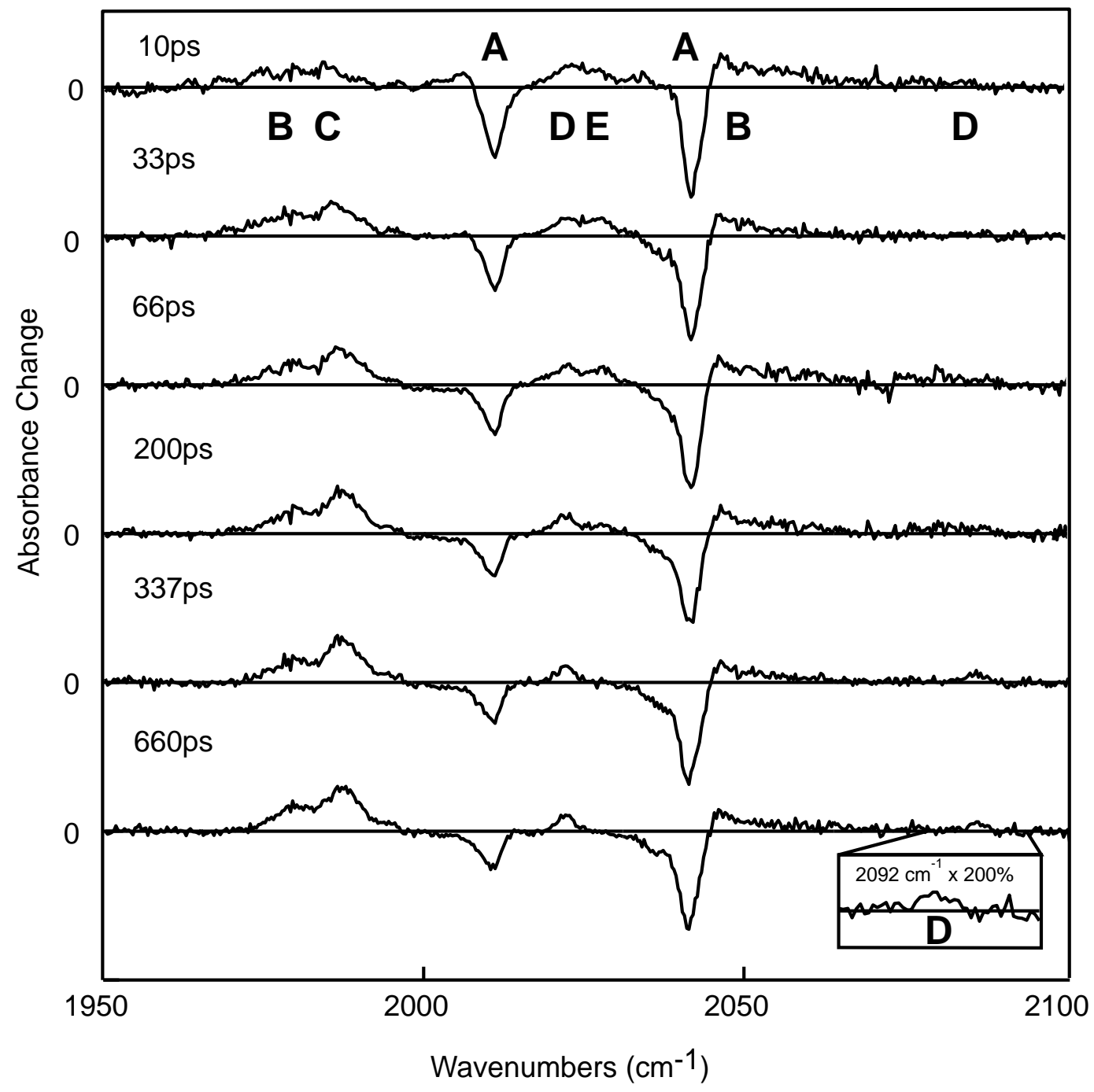

Figure 2.1: Ultrafast transient difference spectra following UV photolysis, at $295 \mathrm{~nm}$, of $\left(\eta^{1}-C_{5} C l_{5}\right) M n(C O)_{5}$ in pentane at time delays of $10 \mathrm{ps}, 33 \mathrm{ps}, 66 \mathrm{ps}, 200 \mathrm{ps}, 337 \mathrm{ps}, 660$ ps. 


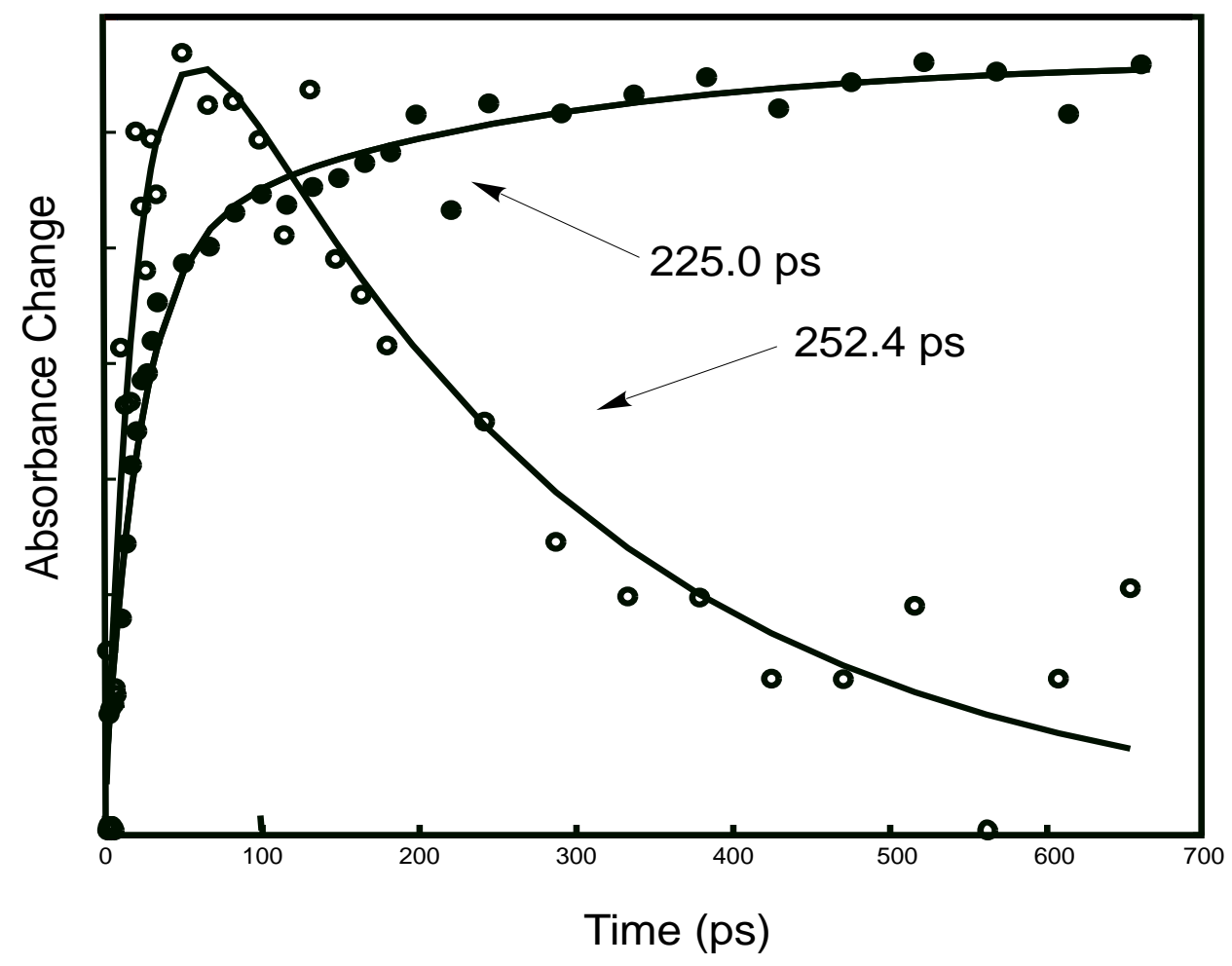

Figure 2.2: Comparison of photoproducts $\mathbf{E}$ and $\mathbf{A}$. Closed circles represent $\mathbf{A}$, open circles E. The solid lines represent the exponential fits to the data. 


\subsubsection{Femtosecond Infrared Spectroscopy: $325 \mathrm{~nm}$ Excitation}

Spectra taken at $325 \mathrm{~nm}$ excitation are shown in Figure 2.3.

With the exception of the change in the relative peak intensities of the photoproducts they are qualitatively the same as the spectra observed at $295 \mathrm{~nm}$ excitation. This change in the relative intensities of the photoproducts is discussed below.

Kinetic measurements taken following $325 \mathrm{~nm}$ excitation differ from the kinetics recorded following $295 \mathrm{~nm}$ excitation. Like the $295 \mathrm{~nm}$ excitation, the photoproducts all show a rise time of less than $50 \mathrm{ps.} \mathrm{However,} \mathrm{the} \mathrm{long-time} \mathrm{decay} \mathrm{of} \mathbf{E}$ is determined to be $178 \mathrm{ps} \pm 7 \mathrm{ps}$ while the recovery of $\mathbf{A}$ at $2010 \mathrm{~cm}^{-1}$ is seen to be $157 \mathrm{ps} \pm 35 \mathrm{ps}$, slightly faster timescales than those seen with $295 \mathrm{~nm}$ excitation.

\subsubsection{Nanosecond Step-Scan FTIR}

The spectra taken using nanosecond step-scan FTIR are in agreement with the femtosecond data except for the appearance of a photoproduct, $\mathbf{F}$, at $1964 \mathrm{~cm}^{-1}, 2020$ $\mathrm{cm}^{-1}$, and $2092 \mathrm{~cm}^{-1} .^{2}$ Kinetic analysis of $\mathbf{F}$ at $1964 \mathrm{~cm}^{-1}$ reveals a rise time of $136 \mathrm{~ns} \pm$ 53 ns followed by constant behavior over the timescale of observation as shown in Figure 2.4 .

The free-energy barrier for the formation of this product can be estimated using the reaction rate derived from transition state theory,

\footnotetext{
${ }^{2}$ Note that $\mathbf{F}$ grows in directly over $\mathbf{D}$. This is confirmed by the kinetic analysis of $\mathbf{F}$ at $2020 \mathrm{~cm}^{-1}$.
} 


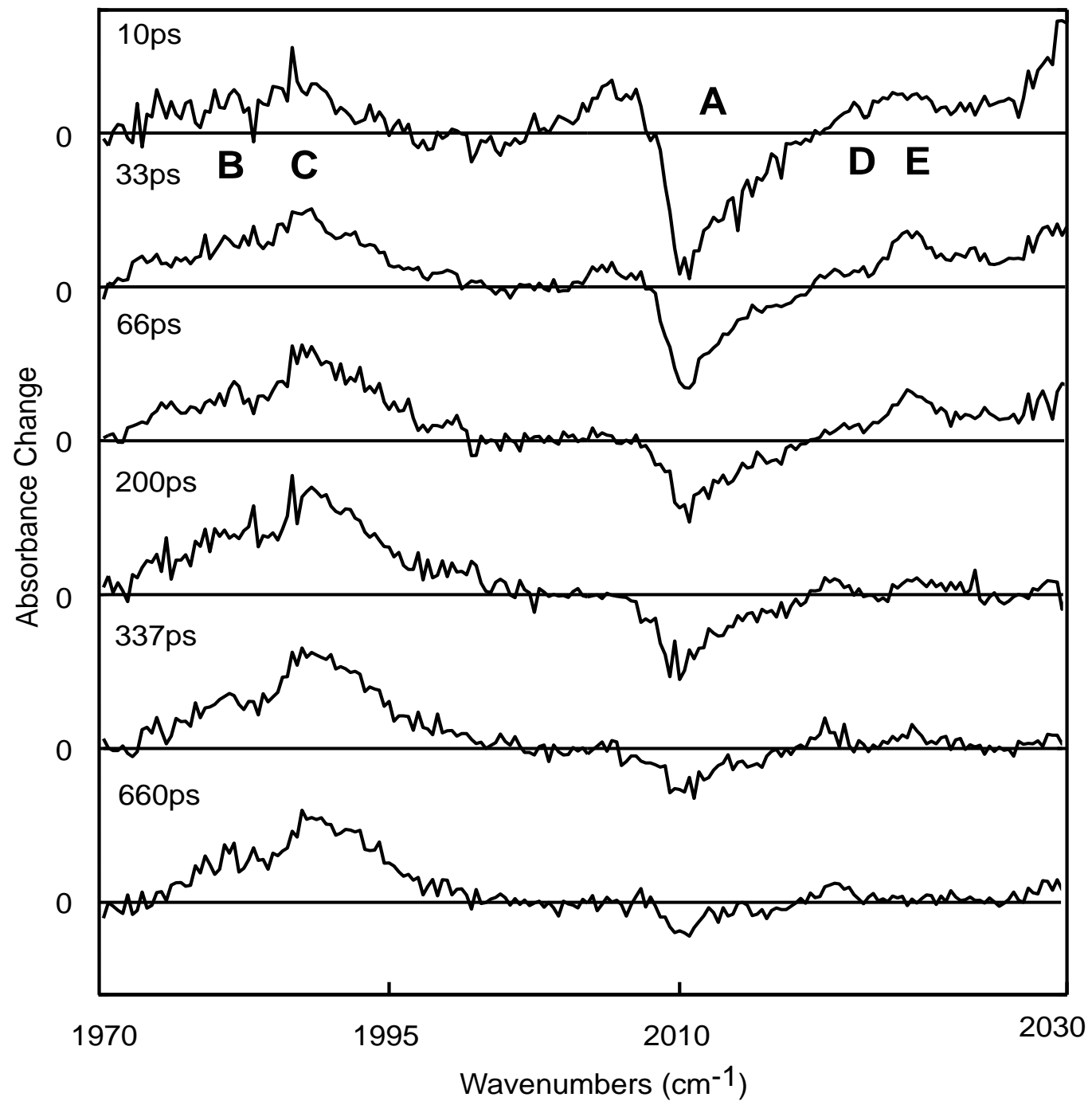

Figure 2.3: Ultrafast transient difference spectra following UV photolysis, at $325 \mathrm{~nm}$, of $\left(\eta^{1}-C_{5} C l_{5}\right) M n(C O)_{5}$ in pentane at time delays of $10 \mathrm{ps}, 33 \mathrm{ps}, 66 \mathrm{ps}, 200 \mathrm{ps}, 337 \mathrm{ps}, 660$ ps. 


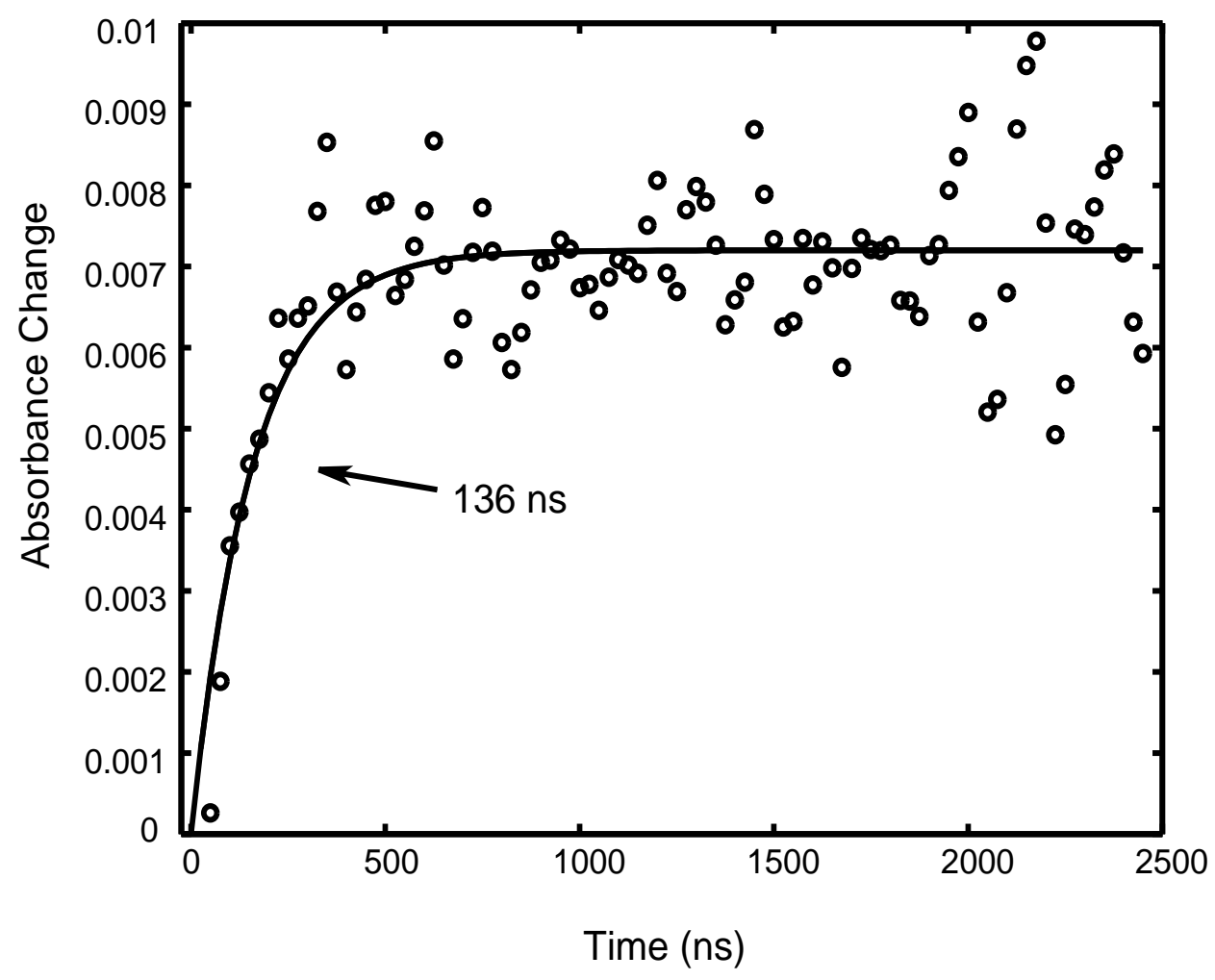

Figure 2.4: Nanosecond kinetics showing the rise time of $\mathbf{F}$ in undecane at $1964 \mathrm{~cm}^{-1}$. Open circles are data points, the solid line represents the exponential fit to the data. 


$$
\frac{1}{\tau}=\frac{k_{B} T}{h} \exp \left(\frac{-\Delta G^{*}}{k_{B} T}\right)
$$

in which $\tau$ is the rise time of the $\mathbf{F}$ measured at $1964 \mathrm{~cm}^{-1}, k_{B}$ is Boltzmann's constant, $\mathrm{h}$ is Planck's constant, $\mathrm{T}$ is room temperature $(298.15 \mathrm{~K})$, and $\Delta \mathrm{G}^{*}$ is the free energy of activation. For a rise time of $136 \mathrm{~ns} \pm 53 \mathrm{~ns}$, transition state theory estimates a free energy barrier of $8.08 \mathrm{kcal} / \mathrm{mol} \pm 0.20 \mathrm{kcal} / \mathrm{mol}$. In comparison, the upper limit for the free energy barrier calculated for the ultrafast kinetics, based on their sub-50 ps rise time, is $3.40 \mathrm{kcal} / \mathrm{mol}$.

\subsubsection{DFT Calculations}

Density functional theory was used to determine possible structures for the intermediate photoproducts observed in the ultrafast and nanosecond spectra. Optimized geometries were formed using the two methods described below. The first method for the formation of an intermediate structure began with the optimized parent species, $\left(\eta^{1}-\right.$ $\left.C_{5} C l_{5}\right) M n(C O)_{5}$. A single carbonyl was then removed and the new structure was optimized. The removal of both equatorial and axial carbonyls was considered. It was found that the lowest energy structure resulted from optimization following the removal of an equatorial carbonyl, in agreement with the studies of Young and Wrighton[37]. The resulting structure, best described as chlorine-activated, is shown in Figure 2.5A. Removal of an axial carbonyl produces an $\eta^{3}$ structure with a relative gas phase energy $+18.8 \mathrm{kcal} / \mathrm{mol}$ 

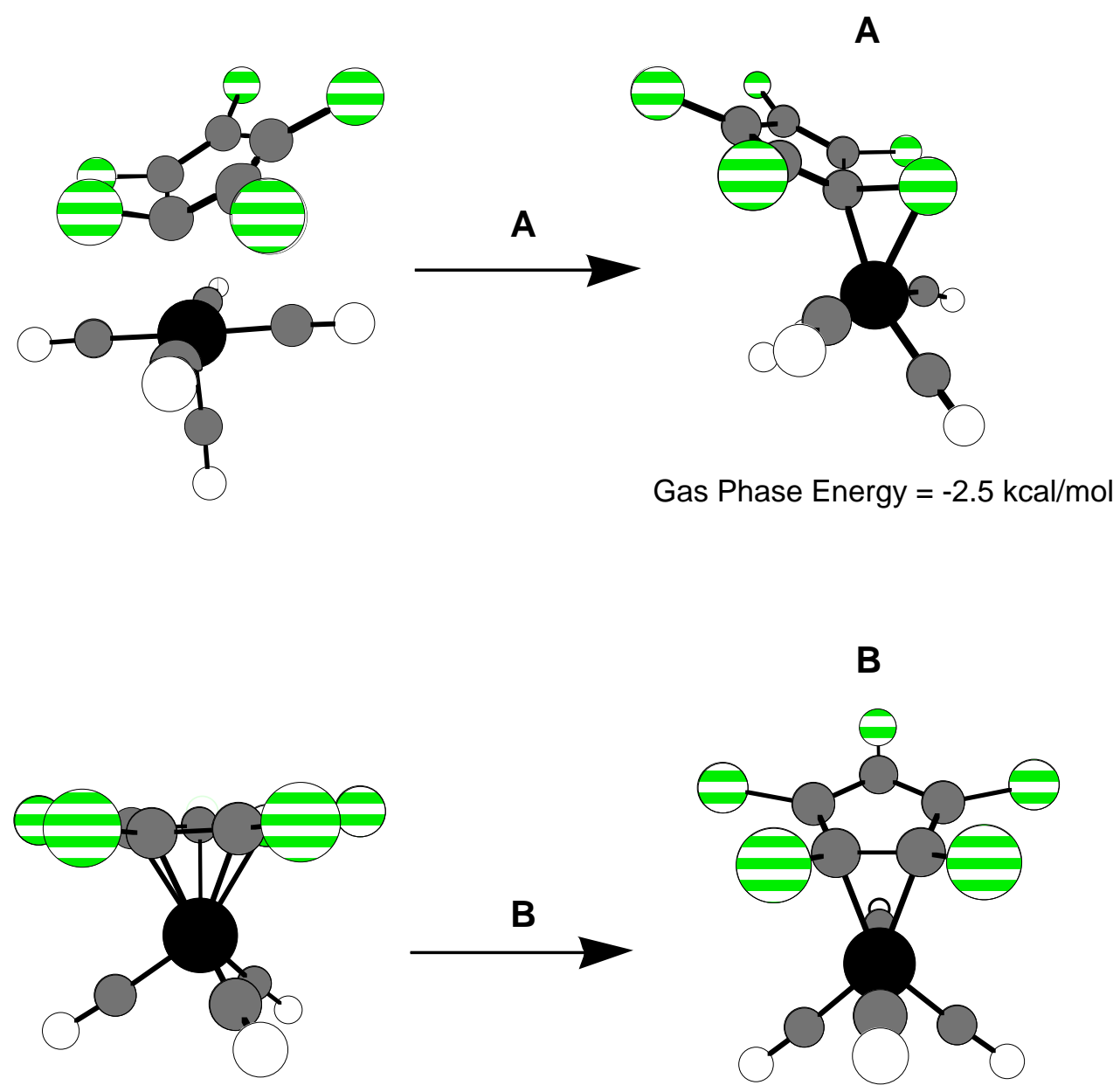

Gas Phase Energy $=0 \mathrm{kcal} / \mathrm{mol}$

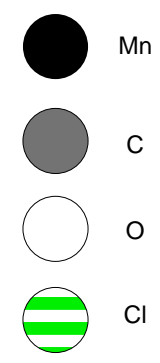

Figure 2.5: Pathway A shows the DFT optimized geometry, best described as chlorineactivated, following the removal of an equatorial carbonyl. Pathway B shows the DFT optimized geometry, best described as $\left(\eta^{2}-C_{5} C l_{5}\right) M n(C O)_{4}$, formed using the method of Veiros. All energies are relative. 
greater than that of the chlorine-activated species.

The second method was based on the procedure of Veiros[52]. This method takes the opposite approach, starting with an optimized $\eta^{5}$ structure. A -2 charge is then added and the structure is optimized again. Veiros had used this method to obtain a structure for $\left(\eta^{3}-C p\right) M n(C O)_{3}^{-2}$. Following the same procedure, but with a chlorinated $\mathrm{Cp}$ ring, results in an $\eta^{2}$ structure, $\left(\eta^{2}-C l_{5} C_{5}\right) M n(C O)_{3}^{-2}$. We extended this method by replacing the -2 charge with an additional carbonyl on the Mn of the optimized $\eta^{2}$ structure. This results in an $\eta^{2}$ structure, $\left(\eta^{2}-C l_{5} C_{5}\right) M n(C O)_{4}$, with a planar ring slipped to an $\eta^{2}$ position as shown in Figure 2.5B. Despite numerous attempts, we have been unable to converge an $\eta^{3}$ structure for a chlorinated Cp ring using this method.

Relative gas phase energies obtained using these methods, listed beneath the corresponding structure, show that the chlorine-activated structure obtained using the first method is $2.5 \mathrm{kcal} / \mathrm{mol}$ lower in energy than the $\left(\eta^{2}-C l_{5} C_{5}\right) M n(C O)_{4}$ structure. Solvation with cyclohexane results in a stabilization of $-19 \mathrm{kcal} / \mathrm{mol}$ for $\left(\eta^{2}-C l_{5} C_{5}\right) M n(C O)_{4}$, a destabilization of $+5 \mathrm{kcal} / \mathrm{mol}$ for the chlorine activated species, and a destabilization of $+3 \mathrm{kcal} / \mathrm{mol}$ for $\left(\eta^{3}-C l_{5} C_{5}\right) M n(C O)_{4}$.

In order to obtain a more general description of the reduced hapticity structures, calculations were performed for tetracarbonyl species in which the chlorinated $\mathrm{Cp}$ ring was replaced with either $\mathrm{Cp}$ or $\mathrm{Cp}^{*}\left[C p^{*}=\left(C_{5} M e_{5}\right), M e=C H_{3}\right]$. Results, shown in Figure 2.6 , indicate that in both cases a lower gas phase energy structure is obtained for the $\eta^{2}$, 
rather than the $\eta^{3}$, species.

As a check of the methods used, $C p_{2} W(C O)_{2}$ has been optimized in the gas phase using a starting structure with the $\mathrm{Cp}$ ring in an $\eta^{2}$ position. It was found that the ring will rearrange to form the $\eta^{3}$ species as is confirmed by the crystal structure. This result verifies the use of DFT for the determination of ring slipped geometries.

\subsection{Discussion}

Excitation of $\mathbf{A}$ with an UV pump beam followed by an IR probe beam reveals the formation of multiple photoproducts, B, C, D, E, F. Several of these photoproducts can be assigned based on previous experiments. Those which have not been previously assigned can be understood in terms of kinetic relationships and structures are proposed using the DFT optimized geometries.

\subsubsection{Assignments and Kinetics}

The assignment of species B with peaks at $1980 \mathrm{~cm}^{-1}$ and $2046 \mathrm{~cm}^{-1}$ is based on the IR peak positions of the compound $\left(\eta^{5}-C_{5} C l_{5}\right) M n(C O)_{3}$ synthesized by Reimer and Shaver[38, 39]. This is a surprising result, as the appearance of a two carbonyl-loss product is unusual for a single photon excitation in the solution phase on the ultrafast timescale. In general, the excess energy from the pump photon will be lost to the surrounding solvent 


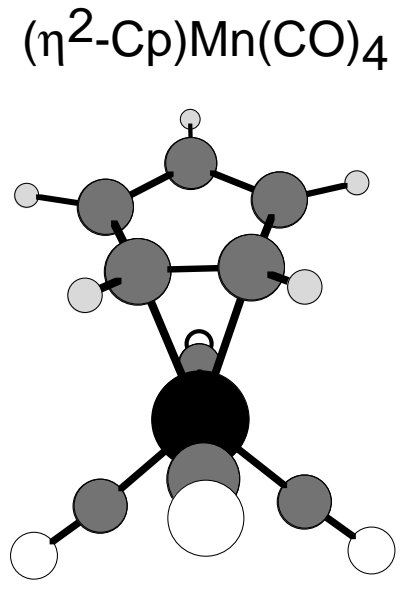

$\mathrm{E}=-20 \mathrm{kcal} / \mathrm{mol}$

$\left(\eta^{2}-\mathrm{Cp}{ }^{*}\right) \mathrm{Mn}(\mathrm{CO})_{4}$

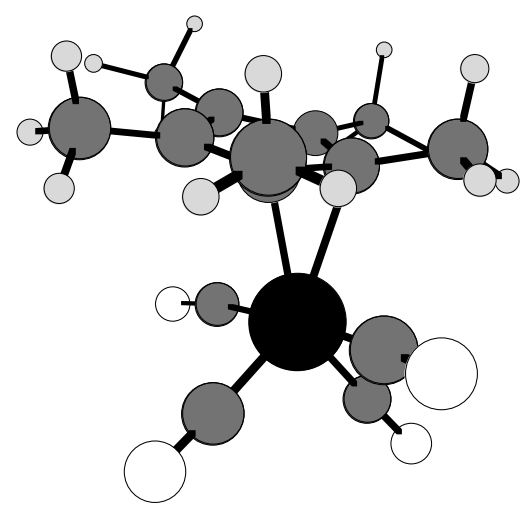

$E=-183 \mathrm{kcal} / \mathrm{mol}$
$\left(\eta^{3}-\mathrm{Cp}\right) \mathrm{Mn}(\mathrm{CO})_{4}$

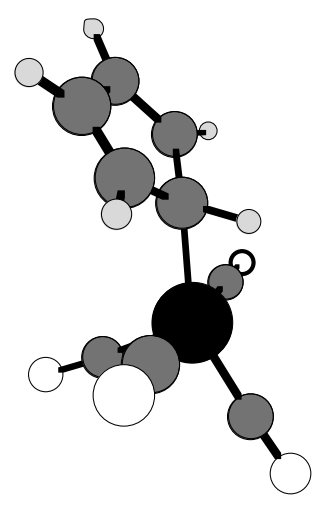

$\mathrm{E}=-1 \mathrm{kcal} / \mathrm{mol}$

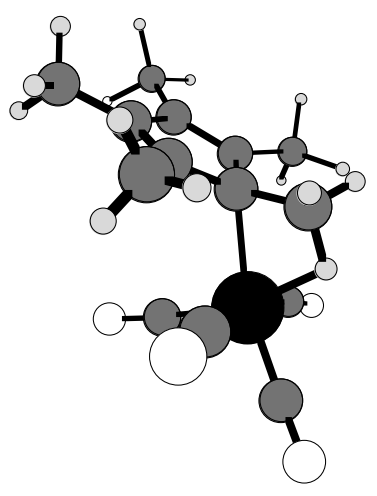

$\mathrm{E}=-1 \mathrm{kcal} / \mathrm{mol}$

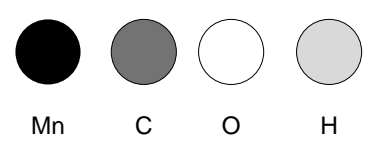

Figure 2.6: DFT optimized structures of $\left(\eta^{2}-C p\right) M n(C O)_{4},\left(\eta^{3}-C p\right) M n(C O)_{4},\left(\eta^{2}-\right.$ $\left.C p^{*}\right) M n(C O)_{4},\left(\eta^{3}-C p^{*}\right) M n(C O)_{4}$ showing relative gas phase energies. 
before a second carbonyl may be lost[53]. ${ }^{3}$

The correlation of the decay of $\mathbf{E}$ with the recovery of the parent species, $\mathbf{A}$, makes it reasonable to consider $\mathbf{E}$ to be an electronic excited state of the parent species which reforms the ground state parent species on a timescale of approximately $250 \mathrm{ps}$ for $295 \mathrm{~nm}$ excitation and 178 ps for $325 \mathrm{~nm}$ excitation. While the possibility exists that $\mathbf{E}$ is a vibrationally excited state of the parent species, it seems unlikely considering the relatively long timescale for recovery compared to approximately 150 ps seen for the vibrational relaxation of organometallic compounds $[55,56,57]$. By varying the wavelength at which we excite the $\left(\eta^{1}-C_{5} C_{5}\right) M n(C O)_{5}$ species we were able to examine the wavelength dependence of this excited state intermediate. Kinetic studies of the excited state decay and parent recovery indicate that this process occurs at a faster rate for lower energy excitation

The radical species, $M n(C O)_{5}, \mathbf{C}$, appears at $1990 \mathrm{~cm}^{-1}$ and is confirmed by earlier experiments of Church, et al.[58]. This product provides a point of comparison for the loss of carbonyl compared to the loss of the chlorinated Cp ring as a function of wavelength. Our results, in agreement with those of Young and Wrighton, show a decrease of the ratio of carbonyl-loss products, $\mathbf{B}$ and $\mathbf{D}$, to the radical $M n(C O)_{5}, \mathbf{C}$, as the wavelength of light increases. ${ }^{4}$

\footnotetext{
${ }^{3}$ The single additional example of two carbonyl-loss in solution had been that of $\mathrm{Fe}(\mathrm{CO})_{5}$. Concurrent work of Snee et. al. has shown that this process is not a true two-carbonyl loss mechansim[54].

${ }^{4}$ The room temperature experiments of Young and Wrighton show that both CO loss and loss of the $\mathrm{C}_{5} \mathrm{Cl}_{5}$, to form the radical species, are possible. Their wavelength dependent studies showed that the loss of $\mathrm{C}_{5} \mathrm{Cl}_{5}$ is not wavelength dependent, while the loss of $\mathrm{CO}$ is greatly affected, ranging from a quantum yield of 0.03 at $436 \mathrm{~nm}$ to 0.30 at $254 \mathrm{~nm}$. This earlier work provides a point of comparison for a discussion of our results. While we were unable to complete a full study of branching ratios, we were able to compare the
} 
The peaks appearing at $1964 \mathrm{~cm}^{-1}, 2020 \mathrm{~cm}^{-1}$, and $2092 \mathrm{~cm}^{-1}$ were attributed to $\left(\eta^{3}-C l_{5} C_{5}\right) M n(C O)_{4}$ by Wrighton and Young. ${ }^{5}$ Their assignment was based on that of the allyl species $\left(\eta^{3}-C_{3} H_{5}\right) M n(C O)_{4}$ which has peaks at $1962 \mathrm{~cm}^{-1}, 1979 \mathrm{~cm}^{-1}, 1996$ $\mathrm{cm}^{-1}$, and $2075 \mathrm{~cm}^{-1}$ at $12 \mathrm{~K}$ in a $\mathrm{CH}_{4}$ matrix[59, 60,61]. It seemed reasonable that a species which was apparently an intermediate in the $\eta^{1}$ to $\eta^{5}$ rearrangement and had a vibrational spectra similar to that of the three carbon allyl would be the $\eta^{3}$ species.

However, on the ultrafast timescale only two peaks at $2020 \mathrm{~cm}^{-1}$ and $2092 \mathrm{~cm}^{-1}$ are observed. It is only on the nanosecond timescale that it is possible to detect a peak at $1964 \mathrm{~cm}^{-1}$. This is a clear indication that there is more than a single step involved in the formation of the final product which Young and Wrighton described as $\eta^{3}$. These results demonstrate the existence of a short-lived intermediate, $\mathbf{D}$, followed by the appearance, on the nanosecond timescale, of a long-lived intermediate, $\mathbf{F} .^{6}$

It is reasonable, based on the assignment of Young and Wrighton, to describe the long-lived intermediate as the ring slipped intermediate. What has yet to be determined are the structures of the intermediate species. First, the structure of the short-lived intermediate relative intensities of the carbonyl-loss and ring-loss photoproducts. A comparison showed that the ratio of $[\mathbf{B}]:[\mathbf{C}]$ changes from 0.62 at $290 \mathrm{~nm}$ to 0.38 at $325 \mathrm{~nm}$. The ratio of $[\mathbf{D}]:[\mathbf{C}]$ shows a similar decrease as the wavelength of light increases, decreasing from 0.36 at $290 \mathrm{~nm}$ to 0.16 at $325 \mathrm{~nm}$.

${ }^{5}$ The spectra of Young and Wrighton showed a peak at $2001 \mathrm{~cm}^{-1}$ which was also attributed to $\left(\eta^{3}-\right.$ $\left.\mathrm{Cl}_{5} \mathrm{C}_{5}\right) \mathrm{Mn}(\mathrm{CO})_{4}$. It is mostly likely obscured by the parent bleach at $2010 \mathrm{~cm}^{-1}$ in our spectra.

${ }^{6}$ Young and Wrighton suggested the possibility that they were unable to observe the initial photoproduct, $\left(\eta^{1}-C l_{5} C_{5}\right) M n(C O)_{4}$, which would be present immediately following the photodissociation of a carbonyl. This could then serve as the first step in the formation of the long-lived intermediate. However it seems unlikely that the rearrangement would occur on a timescale as long as $158 \mathrm{~ns}$ considering that the rearrangement necessary to form $\left(\eta^{5}-C l_{5} C_{5}\right) M n(C O)_{3}$ occurs in less than 1 ps. 
is unknown. Second, the theoretical results described above call into question the description of the ring slipped intermediate as the eighteen electron $\left(\eta^{3}-C l_{5} C_{5}\right) M n(C O)_{4}$ and instead predict the seventeen electron $\left(\eta^{2}-C l_{5} C_{5}\right) M n(C O)_{4}$ to be the more stable structure. These questions are best addressed with the use of DFT calculations.

\subsubsection{Determination of $\mathrm{D}$}

The use of theoretical data for the determination of $\mathbf{D}$ is difficult as no real physical picture exists for its description. Based on the methods for generating DFT geometries, described above, it is possible to consider the chlorine-activated species, shown in Figure $2.5 \mathrm{~A}$, which results from optimization following the removal of an equatorial carbonyl, to be the structure of $\mathbf{D}$. While DFT frequency analysis of all possible structures produces four peaks in our region of observation, it is only the chlorine-activated species which allows the possibility of corresponding to the short-lived intermediate, for which three peaks were observed experimentally. This is based on the spacing of the DFT generated frequencies. Two of the four peaks generated using DFT were predicted to be at $2116 \mathrm{~cm}^{-1}$ and 2118 $\mathrm{cm}^{-1}$, too closely spaced to be resolved experimentally. While this does not confirm the chlorine-activated species as the initial photoproduct, it does provide a possible description of $\mathbf{D}$. 


\subsubsection{Motivation for the Formation of a Seventeen Electron Intermediate}

While the formation of a seventeen electron species proves unusual within a discussion of ring slip, the deviation from the eighteen electron rule is not especially rare. What must be examined is the physical reason for the lower energy of the $\eta^{2}$ compared to the $\eta^{3}$ species. The best way to explore this question is to examine trends of the Group 7 transition metals. As discussed above, it is possible to form both of $\left(\eta^{2}-C l_{5} C_{5}\right) M n(C O)_{4}$ and $\left(\eta^{3}-C l^{5} C^{5}\right) M n(C O)_{4}$. Based on DFT calculations, this is also true for the isoelectronic technetium and rhenium species. Like manganese, technetium and rhenium both result in $\eta^{2}$ species which are lower in energy than the corresponding $\eta^{3}$ species.

The energy difference between the $\eta^{2}$ and $\eta^{3}$ species can be calculated as shown in the table of Figure 2.7. A smaller difference in energy corresponds to a greater ability of the compound to form the unfavorable $\eta^{3}$ species. When plotted against ionization energy, Figure 2.7, it can be seen that there is a strong correlation.

To appreciate the physical value of the result, the different bonding structures of the Cp ring, shown in Figure 2.8, must be considered. For the $\eta^{2}$ structure the bonding of the $\mathrm{Cp}$ ring to the metal can be approximated by bonding to an allene molecule, $\mathrm{C}_{2} \mathrm{H}_{4}$. This bonding structure results in a formal oxidation state of 0 on the metal. The bonding of the $\mathrm{Cp}$ ring in the $\eta^{3}$ form is described as similar to that of an allyl molecule, $C_{2} H_{5}^{-}$. Unlike the $\eta^{2}$ structure, this results in a formal oxidation state of +1 on the metal. 


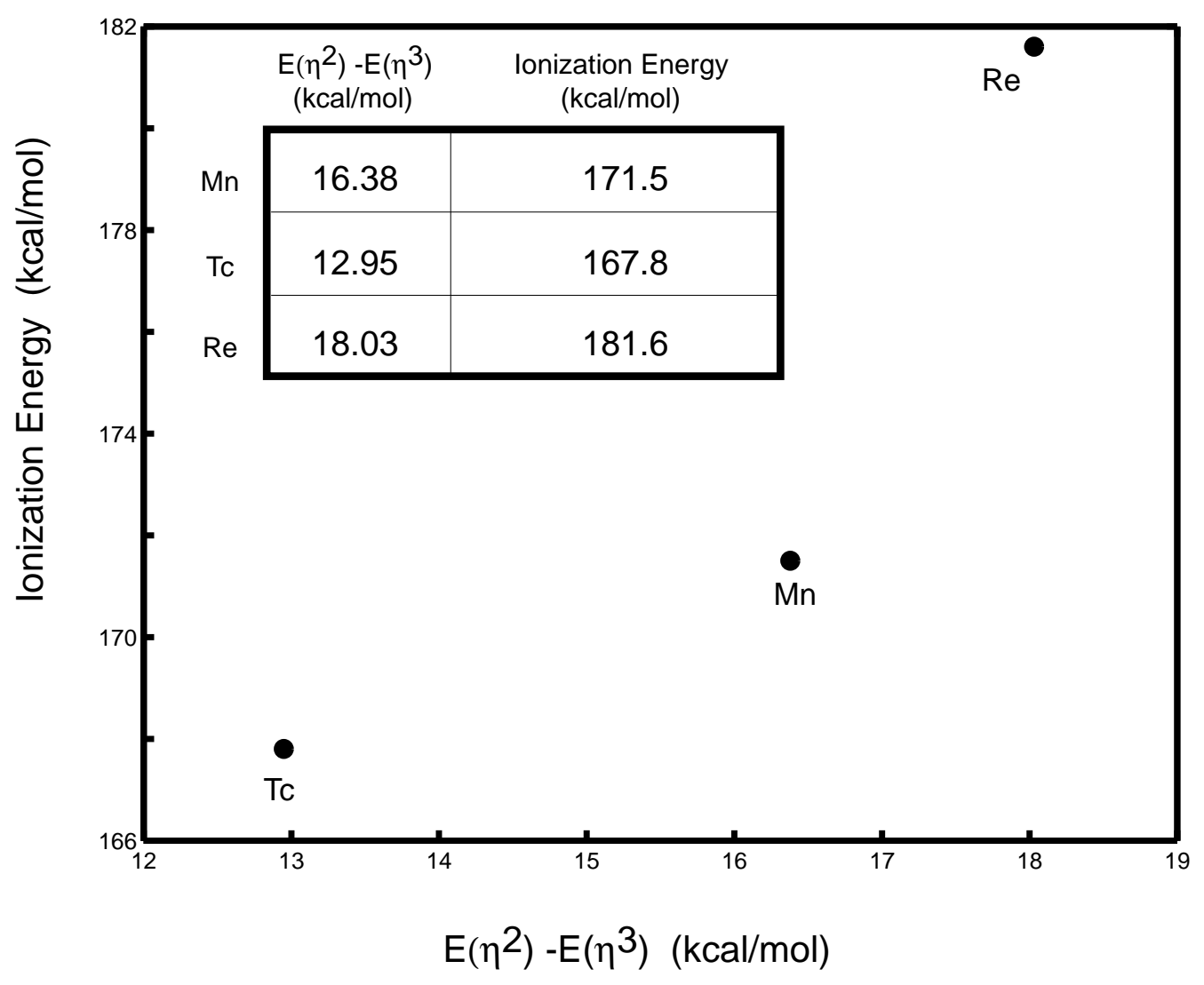

Figure 2.7: Correlation between ionization energy and the gas phase energy difference of $\eta^{2}$ and $\eta^{3}$ cyclopentadienyl species. 

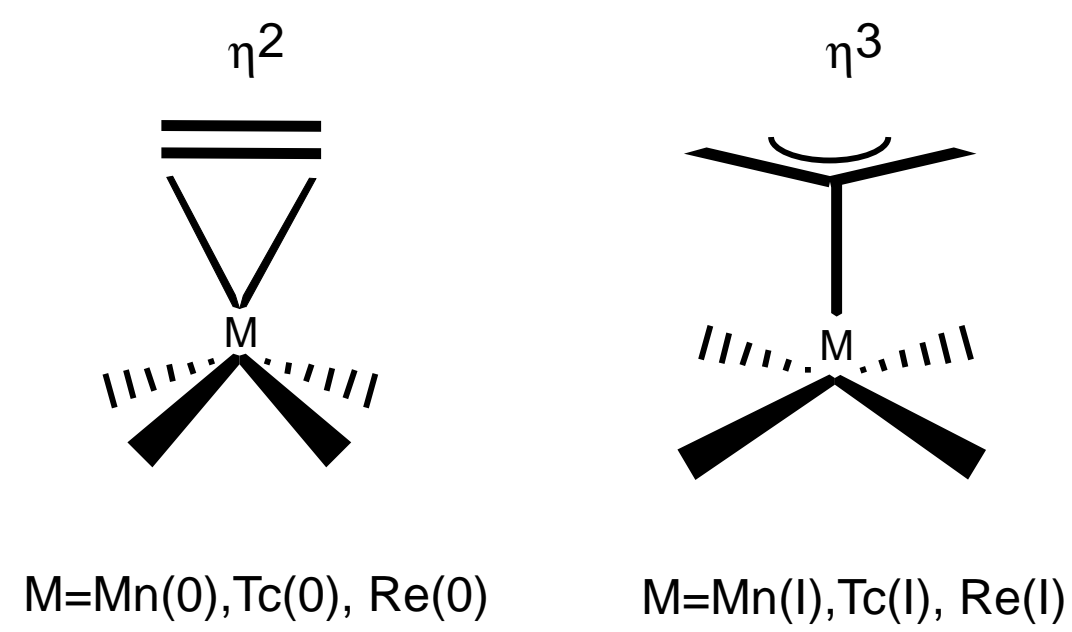

Figure 2.8: $\eta^{2}$ and $\eta^{3}$ Cyclopentadienyl bonding models showing the corresponding oxidation state of the metal.

It is this difference in oxidation which relates to the ionization energy. A metal with a lower ionization energy has a greater ability to form the +1 oxidation state necessary for the formation of the $\eta^{3}$ structure. ${ }^{7}$

\subsubsection{Mechanism}

The overall mechanism of the reverse ring slip of $\left(\eta^{1}-C_{5} C l_{5}\right) M n(C O)_{5}$, shown in Figure 2.9, can be described as follows; the UV excitation of $\left(\eta^{1}-C_{5} C l_{5}\right) M n(C O)_{5}$ results in the fast formation of four species. These four species include $\mathbf{B}$, a two-carbonyl loss

\footnotetext{
${ }^{7}$ This idea can be related to other ring slip structures on a qualitative level. Both the crystal structure and DFT calculation show that $C p_{2} W(C O)_{2}$ forms only an $\eta^{3}$ structure. While the ionization energy of tungsten is $184.0 \mathrm{kcal} / \mathrm{mol}$ it must be remembered that there are two fewer carbonyls on the metal center and for that reason there is less back-donation of electron density to the ligands and more available for oxidation.
} 


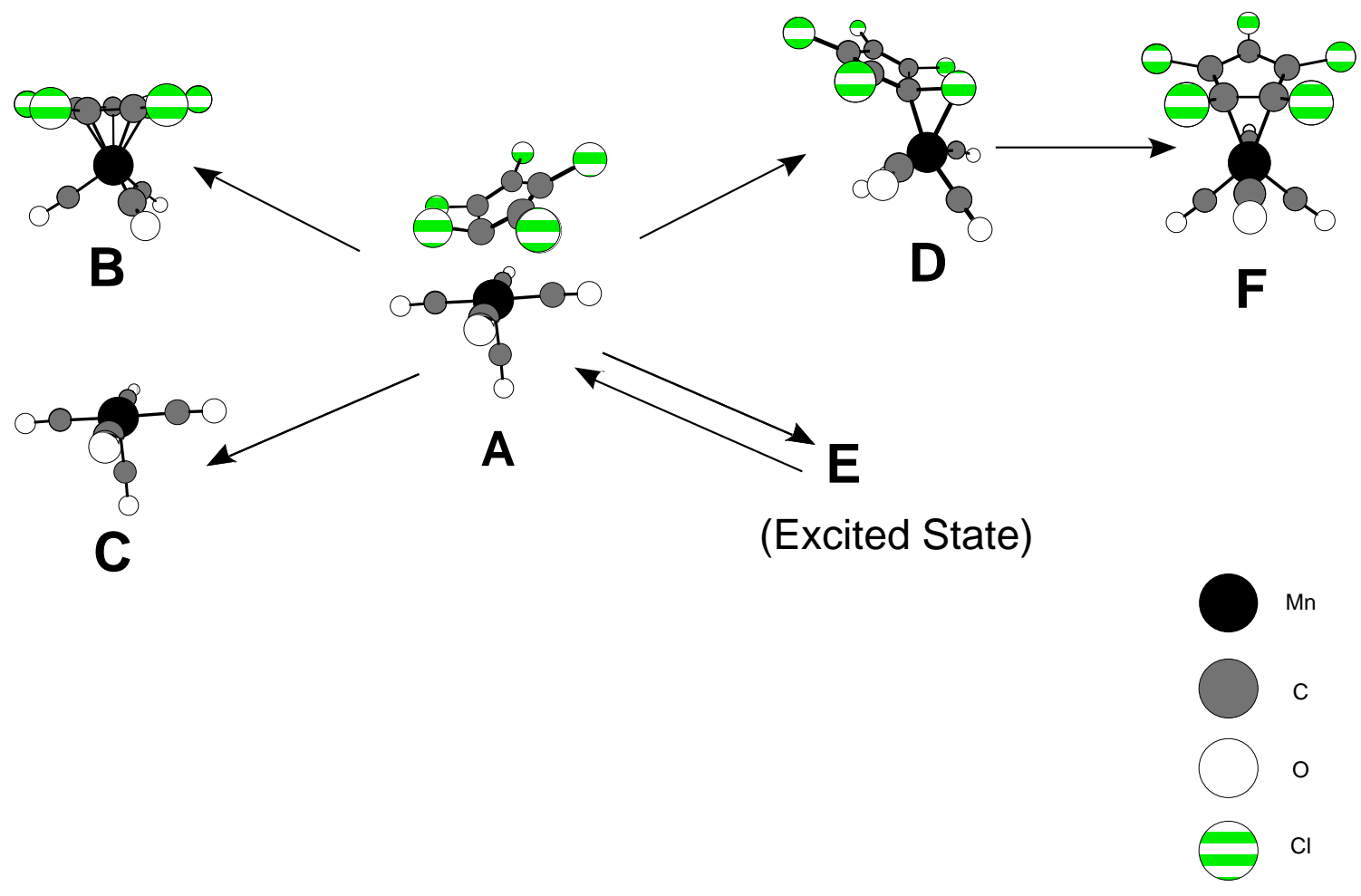

Figure 2.9: Mechanism of ring slip following photoexcitation.

$\left(\eta^{5}-C_{5} C l_{5}\right) M n(C O)_{3}$ product, $\mathbf{E}$, an electronic excited state of the $\left(\eta^{1}-C_{5} C l_{5}\right) M n(C O)_{5}$ parent species, $\mathbf{C}$, the radical $M n(C O)_{5}$, and $\mathbf{D}$, a short-lived intermediate which may be described as chlorine-activated. E reforms the parent species on a timescale of $250 \mathrm{ps}$ for $290 \mathrm{~nm}$ excitation and $178 \mathrm{ps}$ for $325 \mathrm{~nm}$ excitation. $\mathbf{D}$ rearranges to form $\mathbf{F}$, the long-lived $\left(\eta^{2}-C l_{5} C_{5}\right) M n(C O)_{4}$ on a timescale of $136 \mathrm{~ns}$. 


\subsection{Conclusions}

These results allow for the proposal of a detailed mechanism of ring slip on the femtosecond to microsecond timescale. Most interesting is the reconsideration of the assignment of the ring slipped intermediate as a seventeen electron species for Group 7 transition metals. The proposal of a seventeen, rather than eighteen, electron species as the intermediate formed during ring slip is at odds with the fundamental assumption of ring slip. Specifically, ring slip is proposed to maintain an eighteen electron count on the metal center throughout the reaction. Instead it appears that the hapticity of the ring is determined by the ionization energy of the metal. This study has brought into question the formulation of ring slip as mechanism which conforms to the eighteen-electron rule of organometallic chemistry. In addition, it is possible that previous studies which have inferred $\eta^{3}$ species by examining peak shifts in infrared data could be reformulated in terms of a seventeen electron $\eta^{2}$ species. $^{8}$

\footnotetext{
${ }^{8}$ Thanks to H. Frei for assistance with the step-scan FTIR experiments, A.P. Alivisatos for use of an UV-spectrophotometer, and C.B. Moore for the use of an FTIR spectrometer.
} 


\section{Chapter 3}

\section{Investigation of $\mathrm{M}(\mathrm{CO})_{6}$}

$(\mathrm{M}=\mathrm{Cr}, \mathrm{Mo}, \mathrm{W})$ in 1-Hexyne:

\section{Understanding the Mechanism of}

\section{Alkyne Polymerization}

\subsection{Introduction}

The use of metal complexes to catalyze the reactions of organic molecules is a central application of organometallic chemistry. Recent advances have focused on the abil- 


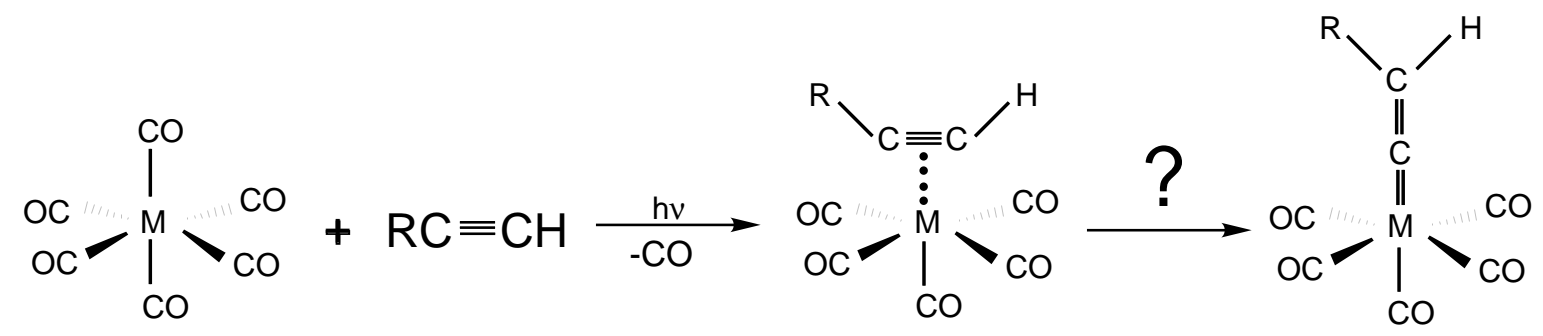

\section{$\mathrm{M}=\mathrm{Cr}, \mathrm{Mo}, \mathrm{W}$}

Figure 3.1: Initial steps of the proposed reaction mechanism for the catalysis of terminal alkynes by Group 6 hexacarbonyls. Photoexcitation results in the dissociation of a carbonyl, the vacant site on the metal is then filled by the coordination of an alkyne solvent molecule which is thought to rearrange to a vinylidenemetal complex.

ity to control the reaction resulting in a selective transformation. One class of selective catalysts is that of organometallic vinylidene complexes[20]. These complexes are the precursor for a variety of rearrangement mechanisms including polymerization, metathesis, and cyclo-oligomerization $[62,63]$. The mechanism for the formation of the vinylidene precursor is thought to proceed through the $\eta^{2}$-coordination of a terminal alkyne followed by the activation of a carbon-hydrogen bond, shown in Figure 3.1. Considering that the initial step is the coordination of the alkyne to the unsaturated metal center, it is important to understand this coordination, as well as the subsequent rearrangement to the vinylidene species.

Previous work has shown that the photodissociation of a carbonyl from an organometallic species in solution occurs on a timescale of less than $100 \mathrm{fs}[14]$. This loss of a carbonyl results in a coordinatively unsaturated metal center capable of interacting with the sur- 
rounding solvent. The coordination of a neighboring solvent molecule to form a looselybound ligand is complete in less than $10 \mathrm{ps}[53]$. For the catalysis of alkynes, it is this coordination of the solvent molecule, in this case a terminal alkyne, which is thought to be the initial step of the reaction. Considering the short timescales involved, it is necessary to examine this mechanism on the ultrafast timescale to determine the rates of formation for the intermediate species. Using an ultrafast ultraviolet-pump infrared-probe laser system, described in Chapter 7, it is possible to dissociate a single carbonyl from an organometallic species and follow the dynamics of the intermediate species in the infrared.

To understand the fundamental aspects of the rearrangement mechanism we have chosen to examine the reaction of $\mathrm{M}(\mathrm{CO})_{6}(\mathrm{M}=\mathrm{Cr}$, Mo, W) in 1-hexyne. The Group 6 metal hexacarbonyl species were the first examples of catalytic vinylidenemetal complexes and these reactions continue to be an active area of investigation[64]. Previous studies of $\mathrm{M}(\mathrm{CO})_{6}$ in low-temperature matrices suggested that the coordination of the alkyne and rearrangement to the vinylidene species occurred following irradiation with ultraviolet light $[65,66,67]$. These experiments agreed with the mechanism proposed in Figure 3.1, but lacked the dynamic information that would be obtained from real-time experiments under ambient conditions. 


\subsection{Results and Discussion}

Results are shown for $\mathrm{Mo}(\mathrm{CO})_{6}$. Similar results were obtained for $\mathrm{Cr}(\mathrm{CO})_{6}$ and $\mathrm{W}(\mathrm{CO})_{6}$

\subsubsection{Femto- to Picosecond Timescale Spectra}

The ultrafast infrared spectra of $\mathrm{Mo}(\mathrm{CO})_{6}$ in 1-hexyne following photoexcitation is shown in Figure 3.2.

The spectra show three peaks at 1927,1960 , and $2080 \mathrm{~cm}^{-1}$ at increasing time delays[56]. These peaks had been assigned to the $\eta^{2}$-alkyne solvated species in low-temperature matrix studies. The peaks at $1927 \mathrm{~cm}^{-1}$ and $1960 \mathrm{~cm}^{-1}$ can be compared to the intermediates observed in similar studies of $\mathrm{Mo}(\mathrm{CO})_{6}$ in hexane. In those studies, a $1934 \mathrm{~cm}^{-1}$ peak was assigned to the A band and the $1962 \mathrm{~cm}^{-1}$ band to the $\mathrm{E}$ band of the hexane-solvated pentacarbonyl species. By comparison, the peaks observed at 1927 and $1960 \mathrm{~cm}^{-1}$ can be assigned to the $\mathrm{A}$ and $\mathrm{E}$ bands of a solvent-coordinated intermediate. The presence of the $2080 \mathrm{~cm}^{-1}$ peak, assigned to the $\eta^{2}$-alkyne solvated intermediate in the low-temperature matrix studies, as well as the slight shift in peak positions compared to the hexane data, suggests that the observed product is the $\eta^{2}$-hexyne solvated intermediate. 


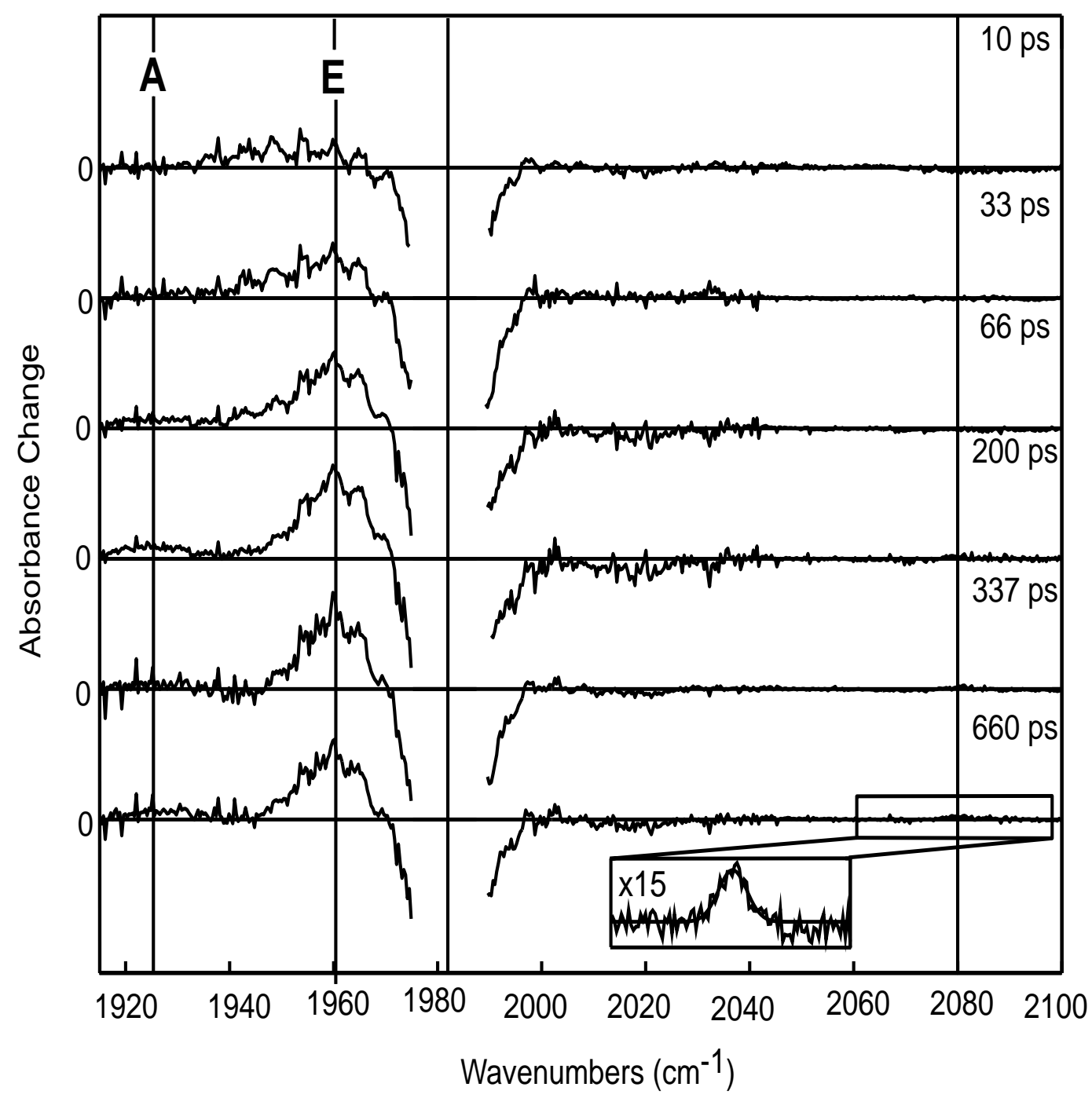

Figure 3.2: Ultrafast time-resolved infrared spectra of $\mathrm{Mo}(\mathrm{CO})_{6}$ in 1-hexyne following photoexcitation. The negative absorption at $1982 \mathrm{~cm}^{-1}$, corresponding to $\mathrm{Mo}(\mathrm{CO})_{6}$, has been cropped for better visualization of the intermediate species at 1927, 1960, and $2080 \mathrm{~cm}^{-1}$. Vertical lines are drawn to emphasize peak positions. 


\subsubsection{Nano- to Millisecond Timescale Spectra}

The ultrafast data show no real kinetic behavior, only a fast instrument response. This observation is in agreement with studies of $\mathrm{CpCo}(\mathrm{CO})_{2}\left(\mathrm{Cp}=\mathrm{C}_{5} \mathrm{H}_{5}\right)$ in hexene which showed the coordination by the double bond of hexene in less than $10 \mathrm{ps}[13]$. In order to study the predicted rearrangement of the $\eta^{2}$-alkyne solvated species, we observed the dynamics on the nano- to millisecond timescale using step-scan and rapid-scan FTIR, described elsewhere[40]. This system operates similarly to the ultrafast experiment using a UV-pulse to dissociate a carbonyl and following the dynamics in the infrared. In these spectra it is possible to observe both the A and E bands at $1933 \mathrm{~cm}^{-1}$ and $1960 \mathrm{~cm}^{-1}$, respectively. Figure 3.3 shows the time-averaged spectrum of $\mathrm{Mo}(\mathrm{CO})_{6}$ in hexyne on the millisecond timescale following photoexcitation.

The corresponding hexane data is plotted with a dashed line to illustrate the shift in frequencies. The weak peak at $2080 \mathrm{~cm}^{-1}$ is not observed, possibly due to its relatively weak oscillator strength. A comparison of the spectra of hexane and hexyne does show convincing evidence that, despite the absence of the $2080 \mathrm{~cm}^{-1}$ peak, the pentacarbonyl is solvated by the triple, rather than single bond which would result in frequencies similar to those observed in the hexane spectrum. Additionally, no rearrangement to the vinylidene species is observed on the nano- to millisecond timescale. 


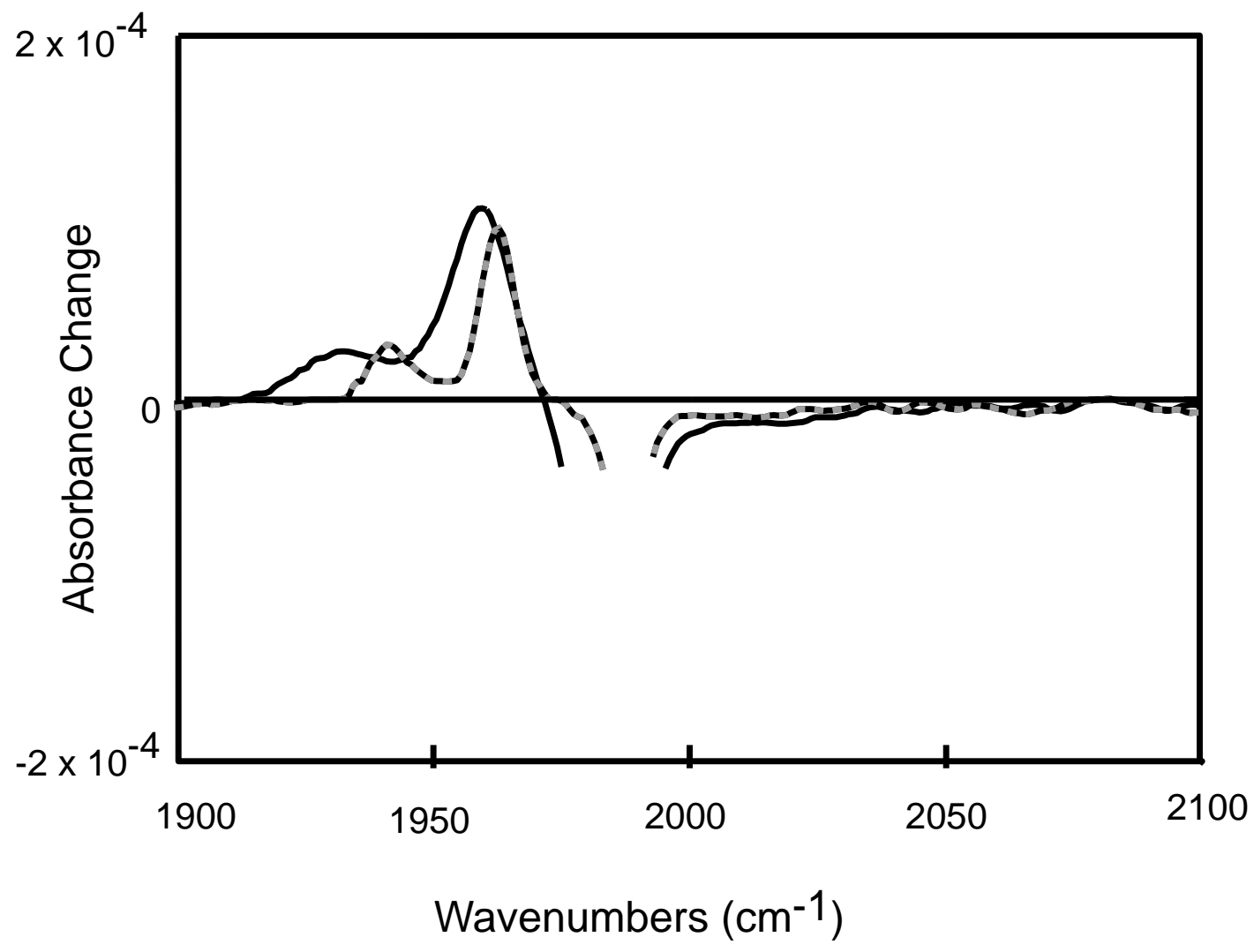

Figure 3.3: Time-averaged millisecond spectra of $\mathrm{Mo}(\mathrm{CO})_{6}$ in 1-hexyne (solid) and hexane (dashed) following photoexcitation. The negative absorption at $1982 \mathrm{~cm}^{-1}$, corresponding to $\mathrm{Mo}(\mathrm{CO})_{6}$, has been cropped for better visualization of the intermediate species at 1933 and $1960 \mathrm{~cm}^{-1}$. 


\subsection{Electronic Structure Calculations: Geometry and Fre- quency}

Although strongly suggested by experimental evidence, it is important to confirm that the intermediate observed experimentally is the triple bond-solvated intermediate rather than an intermediate resulting from solvation by one of the many single bonds of 1-hexyne. Density theoretical calculations were carried out using the B3LYP[42] functional and LACVP ${ }^{* *}[47,48,49]$ basis set within the commercial JAGUAR package[41]. In order to reduce the size of the calculation, propyne and propane were used as models for hexyne and hexane. Comparing the calculated frequencies it is possible to distinguish the two bonding possibilities for a solvent molecule containing a triple bond. The geometry and corresponding frequency of three solvated species are shown in Figure 3.4.

Figure 3.4A shows the triple-bond solvate while Figure 3.4B shows the single bond of the alkyne molecule weakly coordinated to the metal center. Figure $3.4 \mathrm{C}$ shows a propanesolvated species as a point of comparison. The frequencies listed correspond to the calculated values for the $\mathrm{E}$ band of each species. The differences in calculated frequencies are in good agreement with the shifts observed in the millisecond spectra shown in Figure 3.3. Although no scaling factors have been used, it is possible to see that if the single bond of propyne were to interact with the metal center, as is the case in Figure $3.4 \mathrm{~B}$, the E band would be shifted to a higher frequency than that which is experimentally observed. Additionally, 

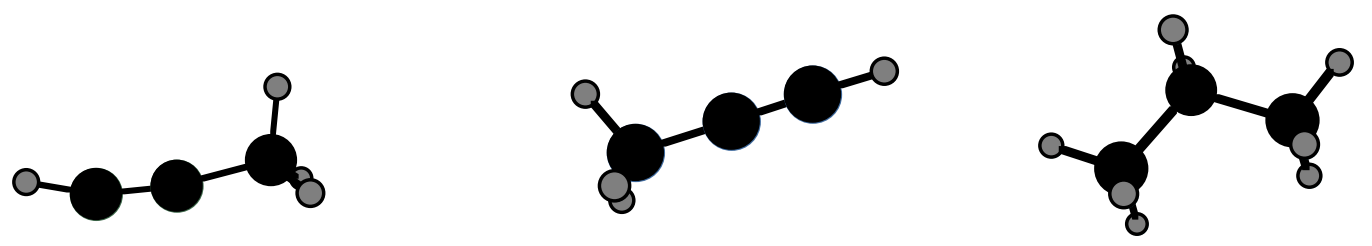

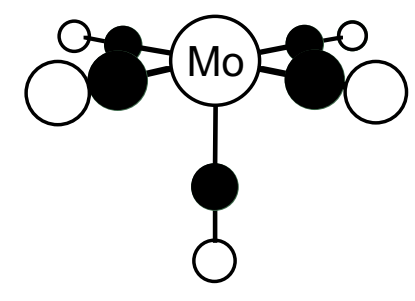

A

$1955 \mathrm{~cm}^{-1}$

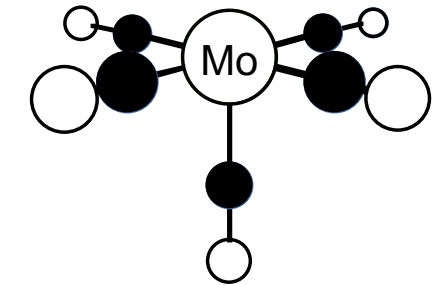

B

$1966 \mathrm{~cm}^{-1}$

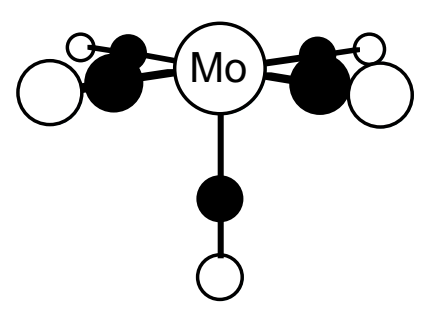

C

$1962 \mathrm{~cm}^{-1}$

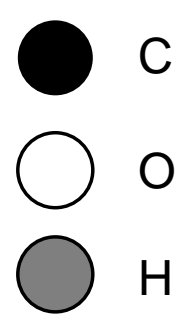

Figure 3.4: DFT calculated geometries and E band frequencies. Geometries A, B, and C correspond to solvation of the metal center by the triple bond of propyne, the single bond of propyne, and propane, respectively. 
calculations of propyne-solvated species show a weak peak at $2141 \mathrm{~cm}^{-1}$ corresponding to the $\mathrm{C}-\mathrm{C}$ triple bond stretch. It is possible that the calculated value is shifted and that the $\mathrm{C}-\mathrm{C}$ triple bond is observed experimentally as the relatively weak peak at $2080 \mathrm{~cm}^{-1}$.

\subsection{Polymerization}

Photolysis experiments carried out with a mercury lamp were monitored by FTIR. A $15.8 \mathrm{mmol}$ solution of $\mathrm{Mo}(\mathrm{CO})_{6}$ in 1-hexyne was prepared and irradiated. The spectrum of mercury contains strong emissions throughout the ultraviolet region providing irradiation comparable to the ultrafast and step-scan experiments. Within five minutes the irradiated solution appears cloudy indicating the start of polymerization. After two and one-half hours, an oily, blue substance has formed. The FTIR, shown in Figure 3.5, reveals a total loss of $\mathrm{Mo}(\mathrm{CO})_{6}$ with concomittant changes in the carbon-hydrogen bending and stretching regions. An NMR spectrum, shown in Figure 3.5, with a range of broad vinylic peaks between 4 and $5 \mathrm{ppm}$, is indicative of an assortment of oligomers of various lengths. In the absence of $\mathrm{Mo}(\mathrm{CO})_{6}$ no reaction is observed. These results confirm the polymerization of 1-hexyne by $\mathrm{Mo}(\mathrm{CO})_{6}$ following exposure to ultraviolet light. 

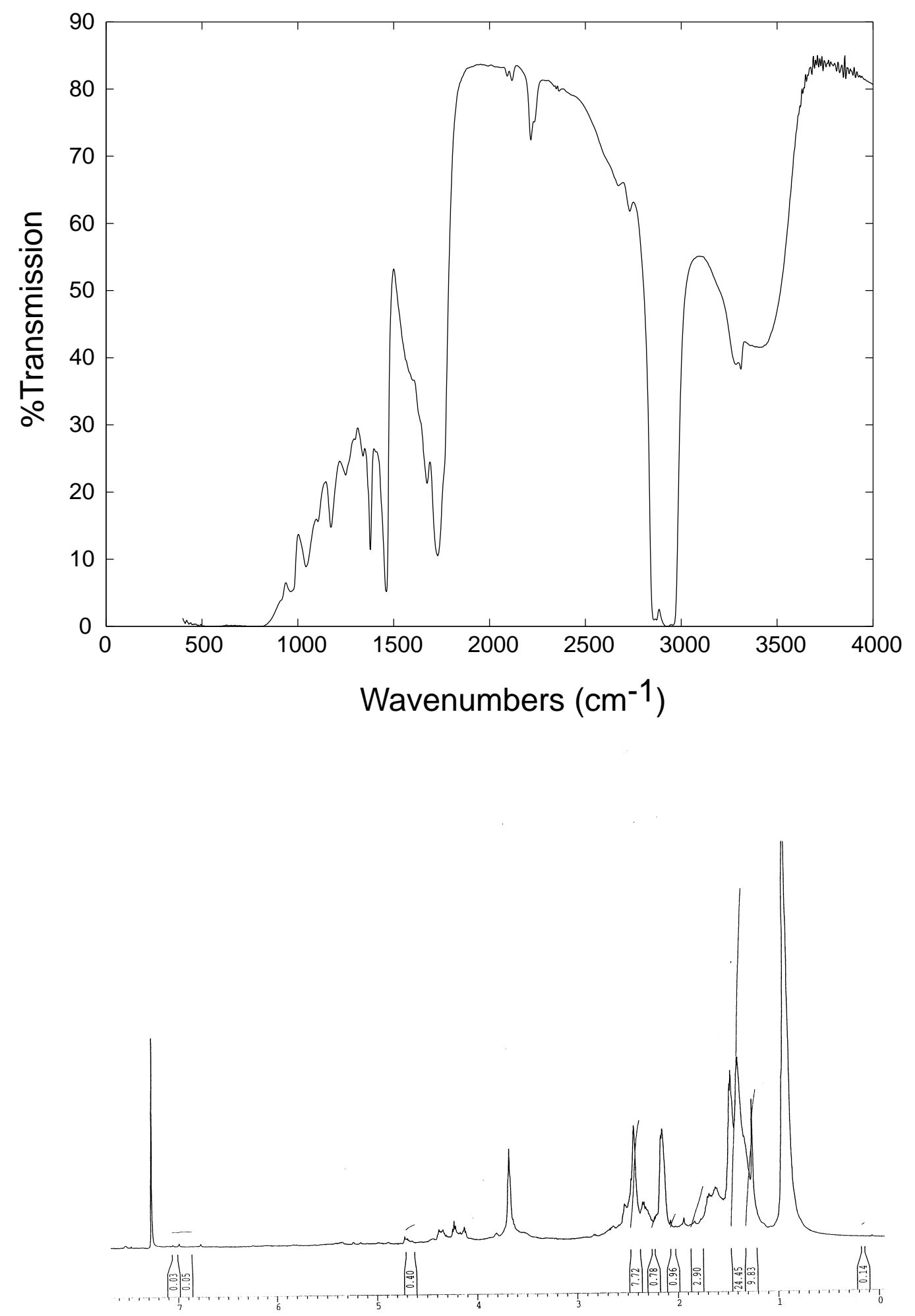

Figure 3.5: FTIR and NMR of the alkyne polymer. 


\subsection{Conclusions}

While it is clear that it is the triple bond which solvates the metal center on the femto- to millisecond timescale, it is unclear why the rearrangement to the vinylidene species does not occur as predicted by the low-temperature studies. As a point of comparison, it has been shown that $\mathrm{Cp} * \operatorname{Ir}\left(\mathrm{PMe}_{3}\right)\left(\mathrm{Cp}^{*}=\mathrm{Me}_{5} C_{5} ; \mathrm{Me}=\mathrm{CH}_{3}\right)$ forms a thermodynamic product through an $\eta^{2}$-interaction with the $\pi$-bonds of ethene and it is possible that a similar interaction occurs for Group VI hexacarbonyls[68]. It is likely that the vinylidene species observed in the low-temperature matrix experiments is actually a two-photon product in which the loss of a second carbonyl, and possibly the coordination of a second alkyne, is necessary to initiate the rearrangement.

The combined use of femto- to millisecond infrared spectroscopy and DFT calculations has demonstrated that catalysis of terminal alkynes proceeds through the coordination of an $\eta^{2}$-triple bond solvated intermediate. This interaction is long-lived and does not rearrange to form the predicted vinylidenemetal species within the timescale of the experiment. It is possible that multiple vacant sites must be created on the metal center for the rearrangement of the 1-alkyne from an $\eta^{2}$-coordinated intermediate to the vinylidenemetal species to proceed. ${ }^{1}$

\footnotetext{
${ }^{1}$ Thanks to Jennifer Krumper for assistance with NMR data.
} 


\section{Chapter 4}

\section{Organometallic Solvation:}

\section{Coordination and Rearrangement}

\section{of Solvent Molecules}

\subsection{Introduction}

Organometallic compounds have proven to be important catalysts for a variety of reactions ranging from industrial processes such as the polymerization of polyethylene to the synthesis of fine chemicals [5]. While the general, thermodynamic, picture of organometallic catalysis is well-understood, the mechanistic details are more difficult to discern. Using 

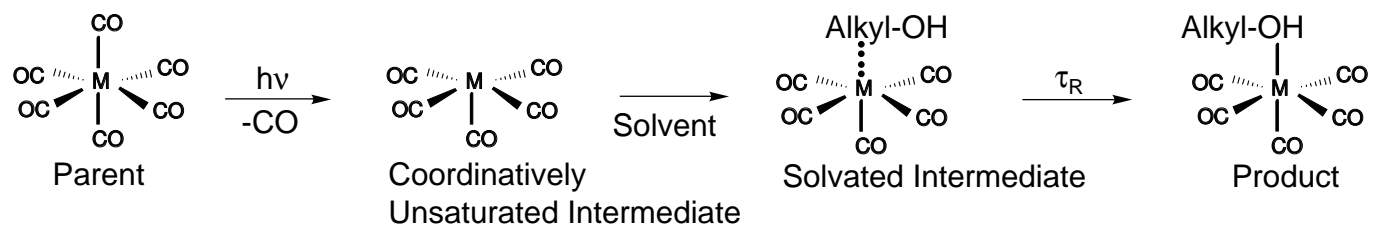

Figure 4.1: General organometallic reaction mechanism initiated by the photodissociation of a carbonyl ligand. While an alcohol solvent molecule is shown above, the reaction mechanism can be applied to a range of solvent systems. The rearrangement of the alcohol solvent molecule is discussed in the text.

ultrafast spectroscopy it is now possible to understand the individual steps through which an organometallic reaction proceeds. This method has been demonstrated for a number of organometallic reactions discussed within this thesis as well as reactions such as carbonand silicon-hydrogen activation[15, 69, 70, 71].

Previous ultrafast studies of organometallic reactions have provided a picture of the steps involved. A general mechanism is shown in Figure 4.1. Briefly, a carbonyl is dissociated from the parent species by a photon of ultraviolet light creating a coordinatively unsaturated intermediate. A neighboring solvent molecule quickly coordinates to the metal center, thereby replacing the electron density lost with the dissociation of the carbonyl. At this point, the reaction mechanism becomes unique. Depending on both the solvent and the organometallic complex involved, the solvent may undergo various rearrangement steps, either intra- or intermolecularly, before forming a final product. Figure 4.1 shows the rearrangement mechanism observed following coordination by an alcohol solvent molecule. 
Alcohol rearrangement has recently been addressed by a comprehensive study of the Group 6 hexacarbonyls in alcohol solvents[15]. These experiments, combined with density functional calculations, suggest an intermolecular mechanism in which a solvent molecule coordinates to the metal center at either a point along the alkyl chain or at the hydroxyl group. If the metal center coordinates at the hydroxyl group the reaction is essentially complete. However, it is more likely that the metal will coordinate along one of the many carbons of the alkyl chain. In this situation, a final product will not be formed. Instead, the solvent molecule will dissociate from the metal center and the coordination will repeat until a hydroxyl group randomly coordinates to the metal.

Energetically, this process has been modeled using a Langevin simulation,

$$
m \frac{d^{2}}{d t^{2}} x=\frac{d}{d t} v=-\frac{d}{d x} V(x)-\gamma m \frac{d}{d t} x+F(t)
$$

which describes the diffusional motion of a particle along a one-dimensional potential and includes a friction term, $\gamma$, and randomly fluctuating force, $\mathrm{F}(\mathrm{t})$. The friction term was obtained by solving the Navier-Stokes equation with "slip" conditions and is dependent on the particle diameter, $\mathrm{d}$, bulk viscosity, $\eta$, and particle mass, $\mathrm{m}$ [72].

$$
\gamma=\frac{2 \pi d \eta}{m}
$$

The potential energy surface is determined by calculating the interaction energy between the 
organometallic complex and the coordinated solvent molecule at each point along the solvent backbone. The interaction energy between the organometallic complex and the hydroxyl group is roughly six times greater than the interaction energy between the organometallic complex and the various coordination sites on the alkyl chain. While the interaction energy varies depending on the specific carbon involved, i.e. $\alpha, \beta$, or $\gamma$, it is always much less than that between the hydroxyl group and the metal center. Figure 4.2 displays a model potential energy surface showing the potential energy as a function of specific bonding position.

This model provides a satisfying picture of the formation of the final, alcohol-coordinated species as an energy minimum. However, it does not provide information regarding the timescale for the initial coordination step. While this process can be assumed to occur "quickly" due to the proximity of the neighboring solvent, the actual timescale of coordination depends strongly on the solvent as well as the organometallic complex.

The interaction between the solvent and the metal center has been well-established. The strength of interaction ultimately depends on the orbital overlap between the metal complex and the solvent. This will depend on features such as the chemical species involved, as seen in the case the $-\mathrm{OH}$ versus - $\mathrm{CH}$ groups, as well as the particular metal involved. It will also depend on the spin state of the organometallic complex. It has been shown that a singlet species will interact more strongly with a solvent molecule than will the corresponding triplet species. This has the overall effect of a faster reaction time for triplet species as they are not hindered by strong interactions with the solvent molecules [54, 73]. 


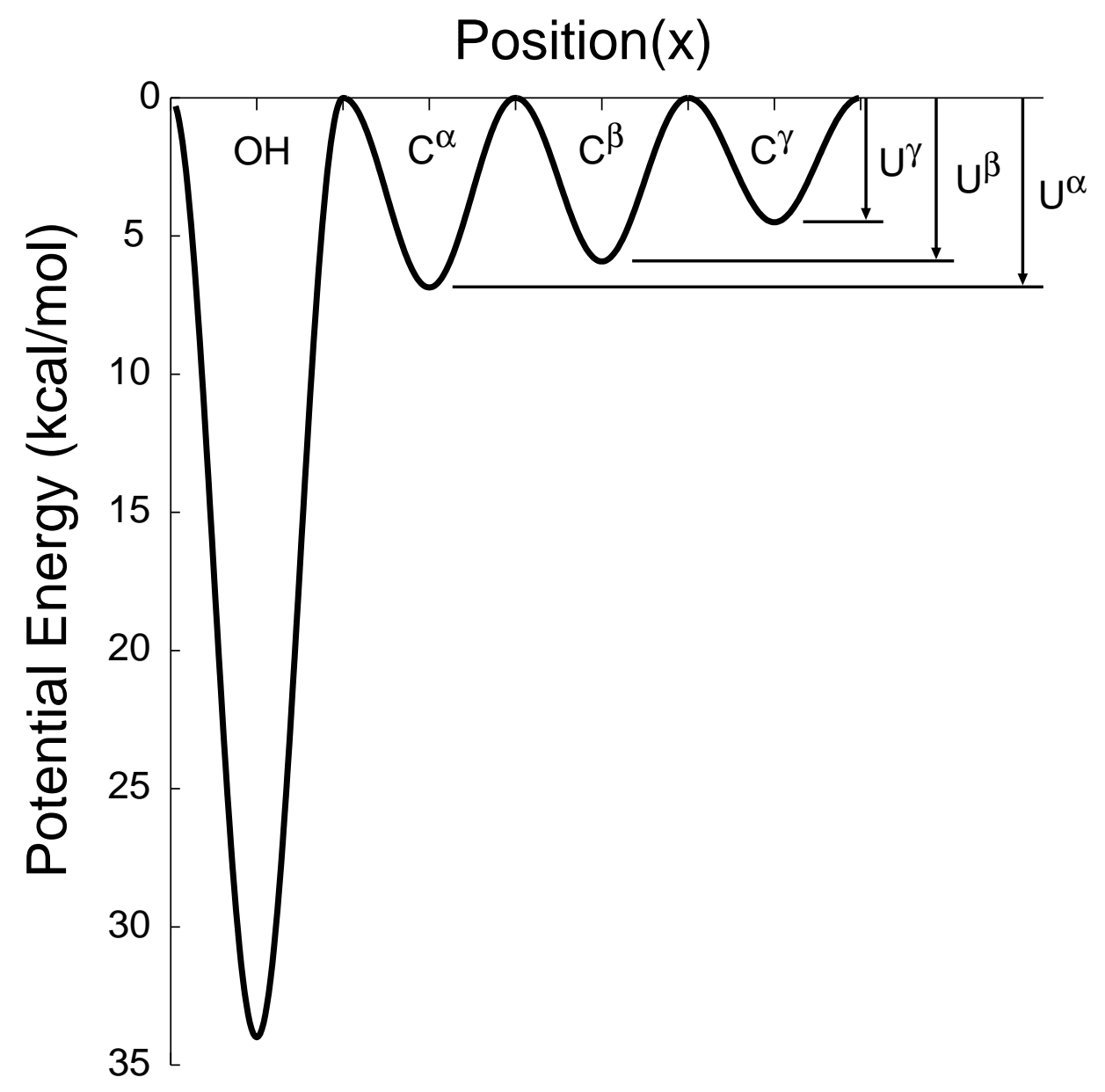

Figure 4.2: Model potential energy surface showing the potential energy as a function of specific bonding position along a solvent backbone. 
What has not been determined is the role that solvent-solvent bonds play in the coordination of a solvent molecule to a metal center. In order to coordinate to the metal center the solvent molecule must also dissociate from the network of solvent bonds. This scenario is best visualized by comparing solvents with hydrogen bonds to those without. A solvent that is part of a hydrogen-bonded network will require more energy to dissociate from the network and bond to the metal center, both during the initial coordination step and and subsequent coordination steps during the course of an intermolecular rearrangement. In addition, the motion of individual solvent molecules will be limited by strong solventsolvent bonds. This limitation should have an affect on the availability of unique binding sites along the solvent molecule. While the importance of solvent-solvent bonds is clear on a conceptual level, it is not clear what the quantitative affect will be for organometallic reaction mechanisms.

In order to address this question experimentally, it is necessary to observe comparable organometallic-solvent systems in which only the solvent-solvent bonding differs. The two requirements that must be met for comparable systems relate to both the organometallic complex and the solvent. In terms of the organometallic complex, it is best to compare identical complexes, thereby removing any question of steric or electronic affects. The requirement of similar solvent systems refers to the energetic picture discussed previously. The formation of a final product is visualized in terms of coordination steps along energy wells of different depths with the final product corresponding to the coordination at the 
solvent site with the greatest well depth. In this case, it is necessary that the energy profile, determined by the interaction energy between the solvent site and organometallic complex, be nearly identical between the solvent systems to be compared. If both the organometallic compound and energy profile can be controlled, it is possible to examine the influence solvent-solvent bonds will have on the coordination steps of a reaction.

Two systems which allow for such a comparison are alcohols and alkynes paired with Group 6 hexacarbonyls. The Group 6 hexacarbonyls, $\mathrm{M}(\mathrm{CO})_{6}(\mathrm{M}=\mathrm{Cr}, \mathrm{Mo}, \mathrm{W})$, provide a simple organometallic species without the complicating factors of more complex ligands. In addition, the differences between each of the metal is well-established. Most important are the similarities between the terminal alcohols and alkynes. As will be discussed below, both have similar interaction energies between individual solvent molecules and the unsaturated metal center. Essentially, the triple bond of the alkyne is equivalent to the hydroxyl group of the alcohol. However, the intermolecular forces of the solvents are quite different, as seen in their vastly different boiling points. It is hoped that the differences in the observed dynamics can be explained by considering the intermolecular forces of the solvent. 


\subsection{Methods}

The methods used for these experiments are identical to those described in Chapters 2 and 7. Alcohol data is taken from previous experiments[15, 74]. Binding energies were calculated using Jaguar[41] or Gaussian98[75] using dummy atoms in fixed geometries to account for basis set errors. Basis sets and functionals are described in Chapters 2 and 3. Zero-point energies were not calculated as they are unlikely to affect the overall trend observed. Methanol and propyne have been used to reduce the size of the calculation. A comparison between methanol and 1-propyne is provided.

\subsection{Results}

Alkyne spectra are shown and discussed in Chapter 3. Alcohol spectra are discussed in the thesis of Kenneth Kotz[74]. Table 4.3 shows the timescale for the formation of the alcohol-solvated species for various solvents.

Table 4.1: Solvents and physical parameters. Sources are discussed in the text.

\begin{tabular}{ccccccc}
\hline \hline Solvent & $\tau_{R}(\mathrm{ps})$ & Boiling Point $\left({ }^{\circ} C\right)$ & Viscosity $(\mathrm{cP})$ & $\tau_{1}(\mathrm{ps})$ & $\tau_{2}(\mathrm{ps})$ & $\tau_{3}(\mathrm{ps})$ \\
\hline ethanol & 45 & 78 & 1.00 & 162 & 6 & - \\
1-propanol & 153 & 97 & 1.11 & 430 & 22 & 2.12 \\
2-propanol & 92 & 82 & 1.72 & 355 & 23.4 & 2.12 \\
1-hexanol & 1800 & 156 & 6.00 & 1210 & 31 & 2.9 \\
1-hexyne & 1 & 71 & .34 & - & - & - \\
& & & & & & \\
\hline \hline
\end{tabular}


Table 4.3 lists these solvents along with the physical parameters considered below. Rearrangement times, $\tau_{R}$, were determined based on exponential fits to the data from Chapter 3 and the thesis of Kenneth Kotz [74]. They correspond to the growth of the alcohol- or alkyne-solvated species as it rearranges from the alkyl-solvated intermediate. Boiling points are taken from Aldrich. Viscosity data is given for $300 \mathrm{~K}$ and is taken from standard sources $[76,77]$. Dielectric relaxation time were measured by Garg and Smyth [78] at $20{ }^{\circ} \mathrm{C}$ with the exception of 2-propanol which was measured by Sato and Buchner [79] at $25{ }^{\circ} \mathrm{C}$. Bond-line formulas showing the carbon skeletons of the various solvents discussed are shown in Figure 4.3 in order to assist in visualization.

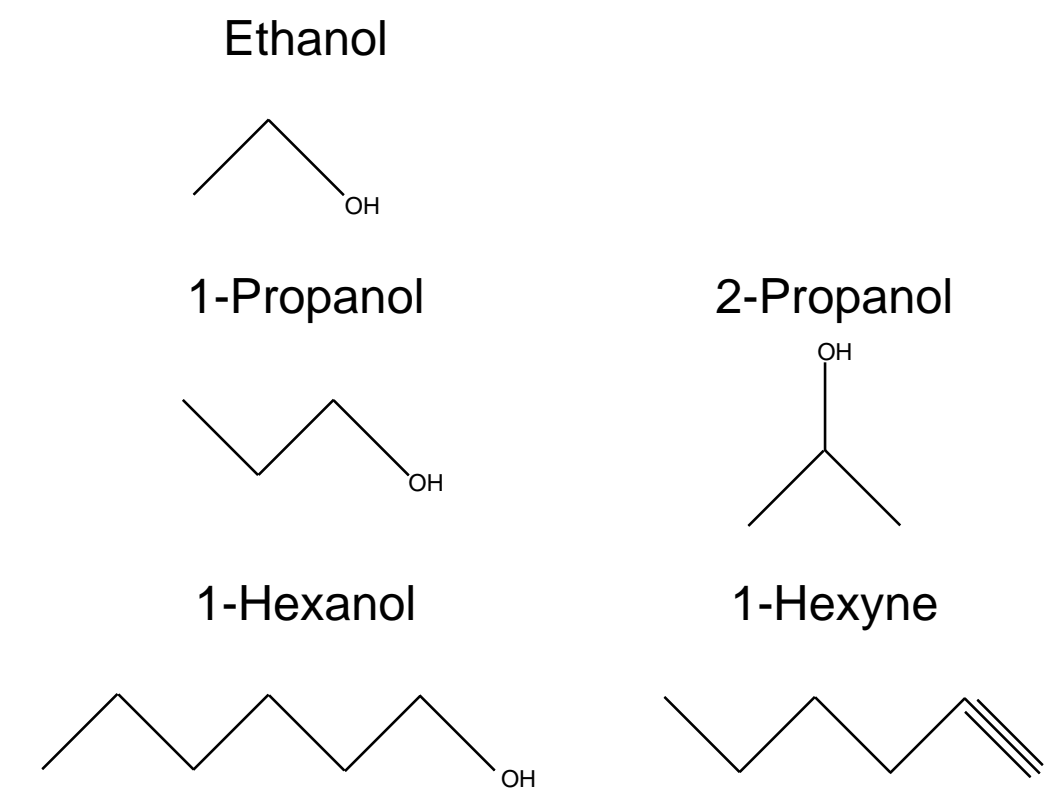

Figure 4.3: Bond-line formulas of solvents discussed in the text. 


\subsubsection{Calculations of Binding Energy}

Geometries and bond energies for $\mathrm{Mo}(\mathrm{CO})_{6}$ complexed to both types of binding sites of methanol and propyne are shown in Figures 4.4 and 4.5. They divide into two categories. Figure 4.4 shows the weakly-interacting binding sites which are similar to a simple alkane solvent.
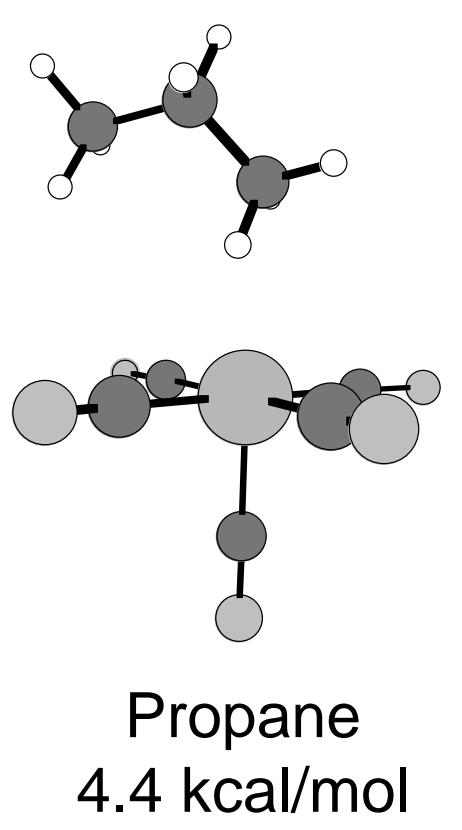
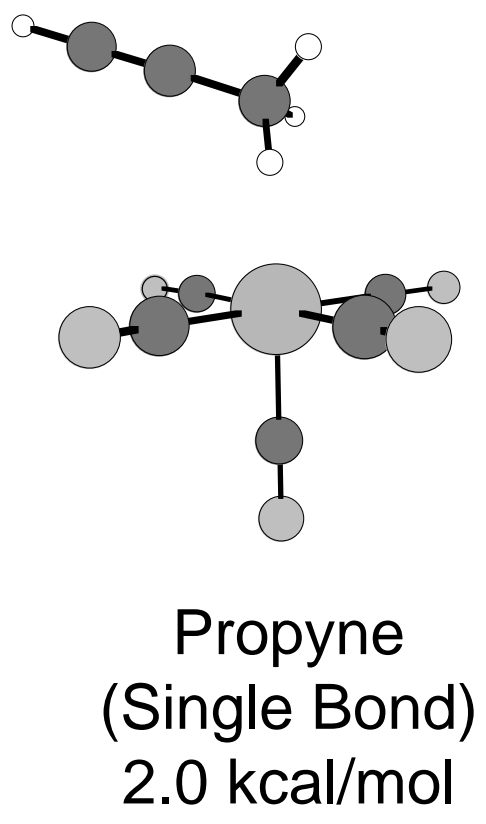
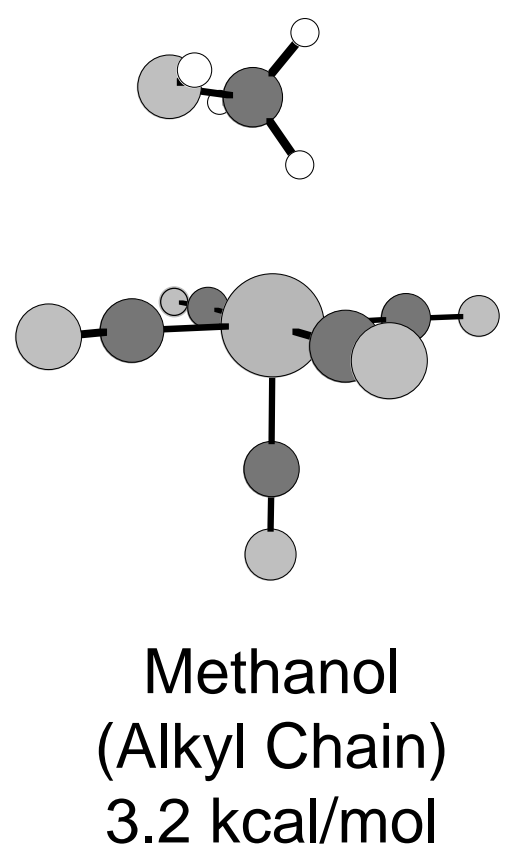

Figure 4.4: Three examples of weakly-bound solvated intermediates include propane, the single bond of propyne, and the alkyl group of methanol. Bond strengths are shown below each structure.

In the case of propyne, this consists of bonding to the single, rather than triple bond. For methanol, the weak interaction is between the metal and the alkyl chain. Propane is 
displayed as a point of comparison.
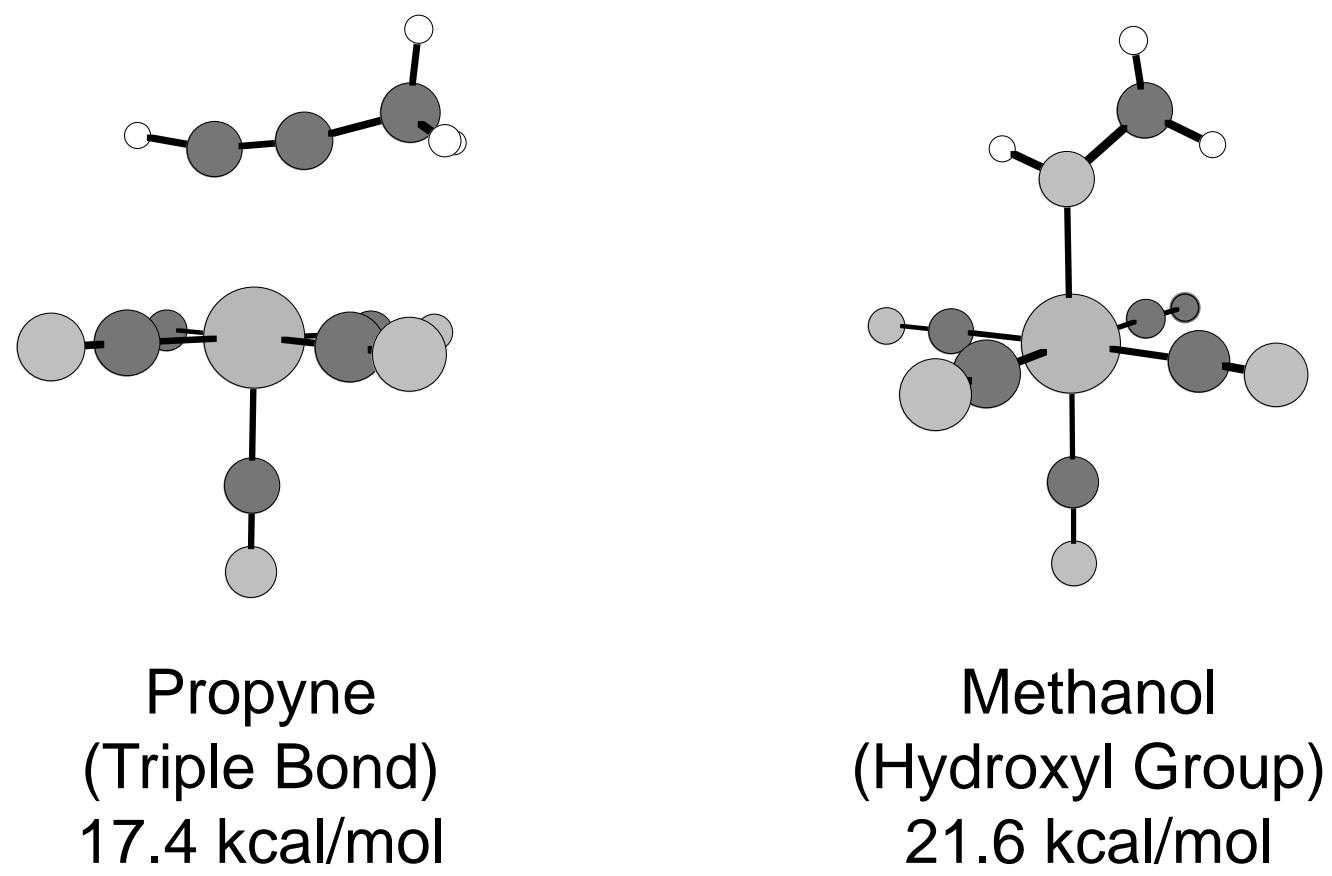

Figure 4.5: Two example of strongly-bound solvated species include the triple-bond of propyne and the hydroxyl group of methanol. Bond strengths are shown below each structure. The propyne complex is a long-lived intermediate and the methanol complex is a product.

The second category includes these the strongly bonding complexes including the interactions of the triple-bond of propyne and the hydroxyl group of methanol with the metal center. As indicated, the bond energies of the metal to the solvent molecules are similar. $\mathrm{Mo}(\mathrm{CO})_{5}$ binds to the hydroxyl group of methanol with an energy of $21.6 \mathrm{kcal} / \mathrm{mol}$ and the triple-bond of propyne with $17.4 \mathrm{kcal} / \mathrm{mol}$. Binding to the alkyl chain is much weaker. For methanol, $3.2 \mathrm{kcal} / \mathrm{mol}$, propyne, $2.0 \mathrm{kcal} / \mathrm{mol}$, and, as a point of comparison, propane, 4.4 
$\mathrm{kcal} / \mathrm{mol}$.

Similar results are obtained for long chain systems shown in Figure 4.6. $\mathrm{Mo}(\mathrm{CO})_{5}$ binds to the hydroxyl group of 1-hexanol with an energy of $17.2 \mathrm{kcal} / \mathrm{mol}$ and the sixth carbon of 1-hexyne with an energy of $4.2 \mathrm{kcal} / \mathrm{mol}$.

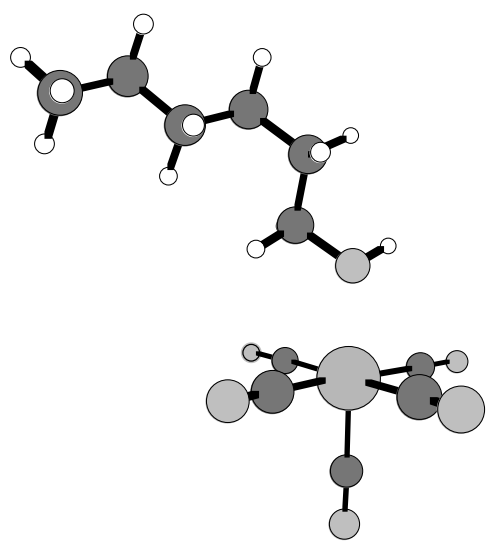

1-Hexanol $17.2 \mathrm{kcal} / \mathrm{mol}$
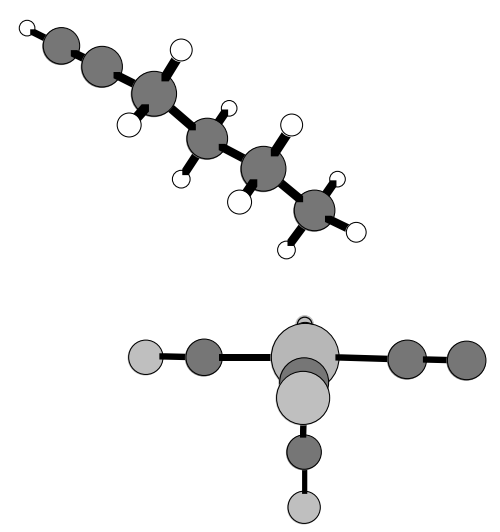

1-Hexyne (Single Bond) $4.2 \mathrm{kcal} / \mathrm{mol}$

Figure 4.6: Full calculations for 1-hexanol and 1-hexyne show bond strengths, listed beneath each structure, that are comparable to the model systems used to reduce the size of the calculation.

\subsection{Discussion}

The product observed in the ultrafast spectra is assigned to $\mathrm{Mo}(\mathrm{CO})_{5}$ complexed with an alkyne solvent based on both low-temperature observations and by comparison to 
similar experiment with hexane as the solvent. The important feature is the fast, less than $1 \mathrm{ps}$, formation of the $\eta^{2}$-solvated intermediate. As a point of comparison, the timescales for the similar product formation of Group 6 hexacarbonyl species are listed in Table 4.3. For 1- hexanol, the timescale is $1.8 \mathrm{~ns}$, more than three orders of magnitude longer than that of 1-hexyne. This result demonstrates the importance of the friction term in the determination of reaction rate considering the similar bond energies for 1-hexanol and 1-hexyne.

Figure 4.7 shows the relationship between rate and viscosity for a single-well barrier as described by Eq. 4.3 using as a barrier height, $\mathrm{E}_{b}=5 \mathrm{k}_{B} \mathrm{~T}, \beta=1$ and $\omega=3 \mathrm{ps}^{-1}$ similar to values obtained for propanol[74].

$$
k=\frac{\sqrt{(\gamma / 2)^{2}+\omega_{b}^{2}}-\gamma / 2}{\omega_{b}}\left[\frac{\omega_{0}}{2 \pi}\right] \exp ^{-\beta E_{b}}
$$

The friction term, $\gamma$, was allowed to vary. While this single-well model does not reproduce the effect observed for heterogeneous potential with multiple dissociation steps, it does provide an estimate of the influence of friction on the rate of the reaction.

The importance of the friction term can best be addressed by considering the intermolecular forces of the solvent. The intermolecular rearrangement mechanism described for alcohol solvents and shown in Figure 4.1 depends on the dissociation of a single solvent molecule from the bulk. The occurs not only during the initial coordination step, but also at each rearrangement step until the energy well, in the form of the hydroxyl group, is encountered. However, it is possible that stronger intermolecular forces, especially hy- 


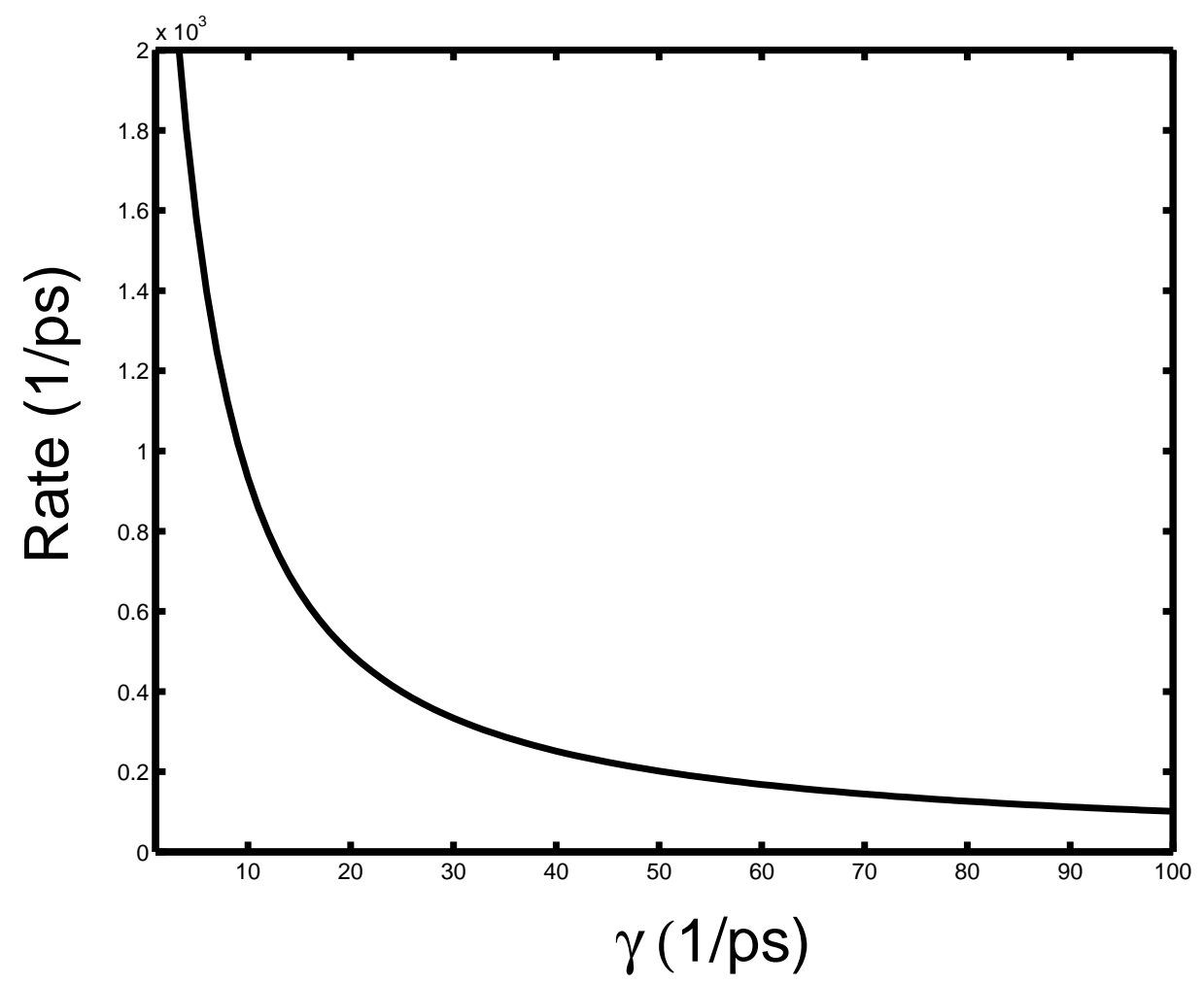

Figure 4.7: Reaction rate as a function friction as described by Eq. 4.3. Friction is calculated as described in the text.

drogen bonds, within the solvent could produce an energetic barrier to the formation of a solvated-intermediate. There are two initially apparent contributions to this energy barrier. The first contribution is due to strong intermolecular forces preventing the dissociation of a single solvent molecule from the bulk to form the metal-solvent bond. The second contribution would be statistical in nature as a strong intermolecular forces would prevent the fast rearrangement of the solvent necessary for the exposure of new binding sites. 


\subsubsection{Intermolecular Forces-Dissociation of a Solvent Molecule from the}

\section{Bulk}

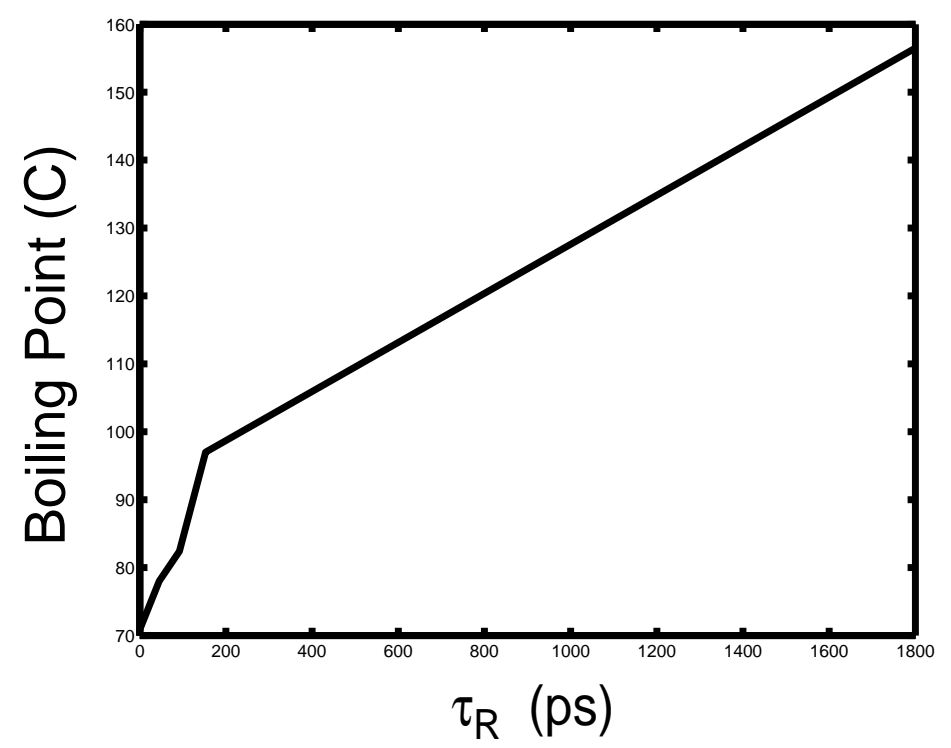

Figure 4.8: Relationship between boiling point and rearrangement time.

On the most basic level, intermolecular forces can be measured as a function of boiling point. Figure 4.8 shows the rearrangement times of the various solvents discussed as a function of boiling point. While no strong relationship is observed, it is interesting to note that the boiling points and rearrangement times of 1- and 2-propanol are at least correlated. 


\subsubsection{Dielectric Relaxation-Rearrangement of Solvent Molecules}

The Langevin simulations discussed above depends on a friction term, $\gamma$, that used bulk viscosity to determine the rate at which the metal center moved between binding sites. This term suffers from a number of drawbacks. Essentially, viscosity is a macroscopic parameter that is most correctly used to describe diffusion-limited processes of which ultrafast dynamics are not an example. Figure 4.9 A shows the relationship between rearrangement time, $\tau_{R}$, and viscosity for the solvents discussed. Plots such as these have been used to demonstrate the need for binding-site-specific models for the description of rearrangement times. Previous experiments have demonstrated the formation of a solvent cage around the reactive species. In this case, viscosity proves to be an insufficient parameter for the description of the system as caging dynamics will be the dominant process at early times. The true test of this parameter would require that the Langevin simulations be carried out for the 1-hexyne potential energy surface and viscosity and compared to experimentally determined rates.

In terms of solvent rearrangement, it is useful to consider dielectric relaxation measurements which have observed the relaxation of alcohols on three separate timescales. The first, $\tau_{1}$ has been attributed to the motion of the molecular aggregate including the breaking of hydrogen bonds and rotation of the hydroxyl group . The second, $\tau_{2}$, consists of the rotation of individual solvent molecules. The third, $\tau_{3}$, involves the rotation of the 

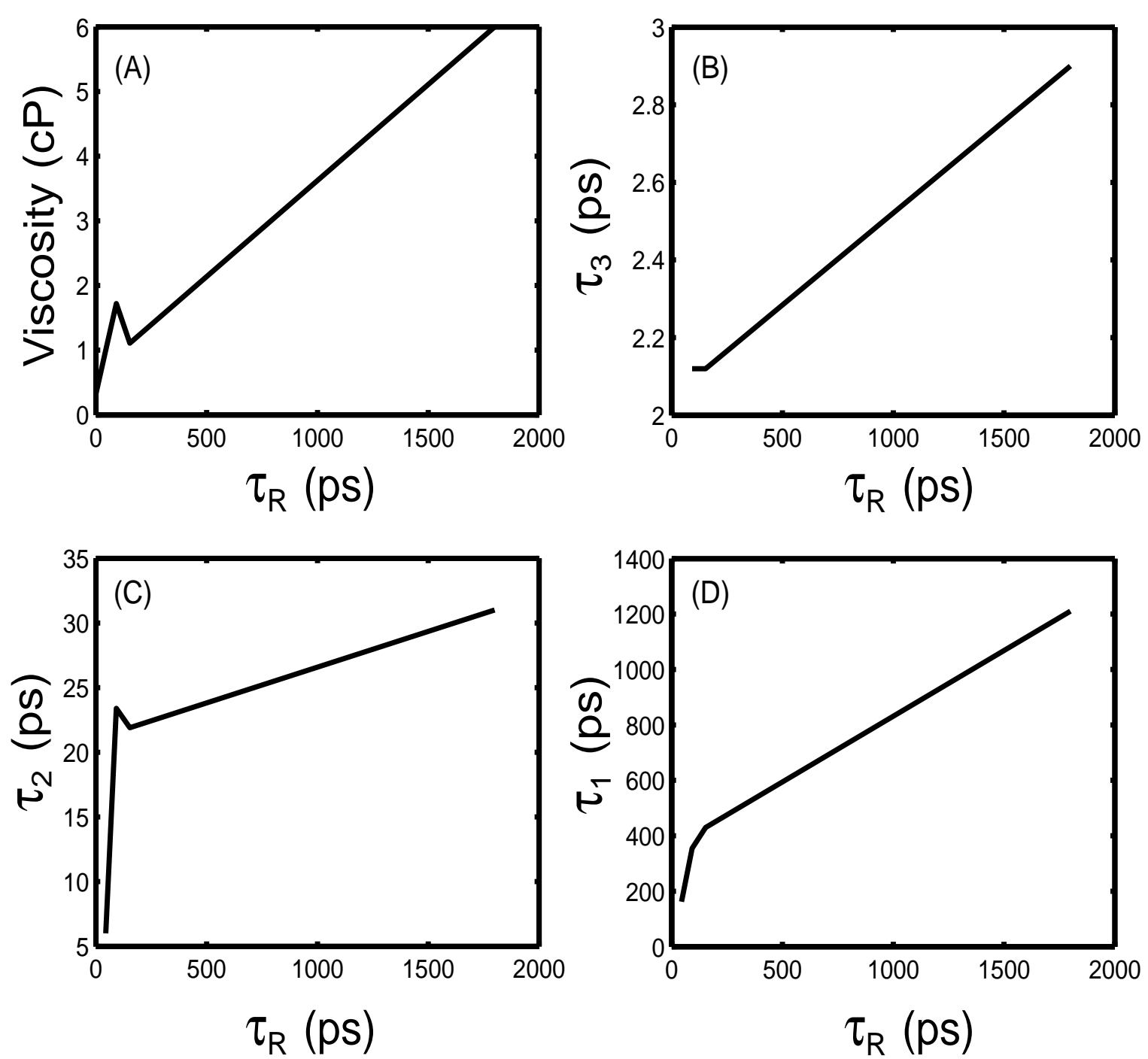

Figure 4.9: Alcohol rearrangement times as a function of viscosity and dielectric relaxation rates. 
hydroxyl group around the carbon-oxygen bond. Figures 4.9 B,C, and D, respectively, show the correlation between the time to form the alcohol-bonded final product, the rearrangement time $\tau_{R}$, and the three relaxation regimes. These values are thought to most closely relate to the reorientation of the solvent cage and should provide a good measure of new binding sites generated.

\subsection{Conclusions}

While Langevin simulations incorporating specific binding site energies and bulk viscosity have proven successful for the calculation of alcohol product formation, it is unclear if this model can be extended to other solvent systems. It is likely that a closer consideration of the intermolecular solvent forces will provide a model possible of describing the experimentally determined alkyne-binding rate. Most importantly, the rearrangement of the individual solvent molecules must be considered in terms of the generation of new binding sites for the metal center. ${ }^{1}$

\footnotetext{
${ }^{1}$ Thanks to Dor Ben-Amotz for helpful discussions.
} 


\section{Chapter 5}

\section{Methods: An Overview of}

\section{Experimental and Computational}

\section{Techniques}

\subsection{Organometallic Photochemistry}

To obtain a complete understanding of organometallic reaction dynamics it is nec-

essary to observe the reaction from initiation to completion. As described previously, the timescales involved in these reactions can be as short as femtoseconds. For this reason, it is important to address these reactions with a method capable of resolving the ultrafast 
dynamics. It has been shown that an ideal method for the study of such reaction dynamics is ultrafast ultraviolet-pump infrared-probe spectroscopy. ${ }^{1}$ Ultraviolet-pump infraredprobe spectroscopy utilizes two separate regions of the electromagnetic spectrum in which organometallic complexes typically absorb radiation. The pump beam is used to initiate the reaction while the probe beam follows the resulting dynamics.

Shown in Figure 5.1 are UV-Vis spectra of pentachlorocyclopentadienyl manganese pentacarbonyl, $\eta^{1}-\left(C_{5} C l_{5}\right) M n(C O)_{5}$, and dimanganese decacarbonyl, $\mathrm{Mn}_{2}(C O)_{10}$. The spectrum of $\eta^{1}-\left(C_{5} C l_{5}\right) M n(C O)_{5}$ shows two absorptions at $225 \mathrm{~nm}$ and $300 \mathrm{~nm}^{2}$ These features are representative of other organometallic species and correspond to a metal-toligand charge transfer (MLCT) bands. MLCT bands arise from the transfer of charge from the metal to the ligand orbitals, in this case to the $\pi^{*}$ orbitals of the carbonyl ligands[37]. Ligand field (LF) transitions between d-orbitals of the metal are most likely present as well, but are obscured by the much stronger MLCT transitions. As a point of comparison, the UV-Vis spectrum of dimanganese decacarbonyl, $\mathrm{Mn}_{2}(C O)_{10}$, is also shown in Figure 5.1. This spectrum shows a broad absorption in the ultraviolet region similar to that seen for $\eta^{1}-\left(C_{5} C l_{5}\right) M n(C O)_{5}$ and also corresponds to a MLCT excitations. In addition, $\mathrm{Mn}_{2}(C O)_{10}$ has a broad absorption tailing off near $400 \mathrm{~nm}$. This broad absorption is attributed to two excitations, a peak at $374 \mathrm{~nm}$ and a shoulder at $390 \mathrm{~nm}$, both ligand field

\footnotetext{
${ }^{1}$ For unlimited references on ultrafast UV-pump IR-probe spectroscopy of organometallic complexes see C.B. Harris or E.J. Heilweil.

${ }^{2}$ The odd feature at $360 \mathrm{~nm}$ is an example of Wood's anomaly and is due to the diffraction grating of the spectrophotometer.
} 

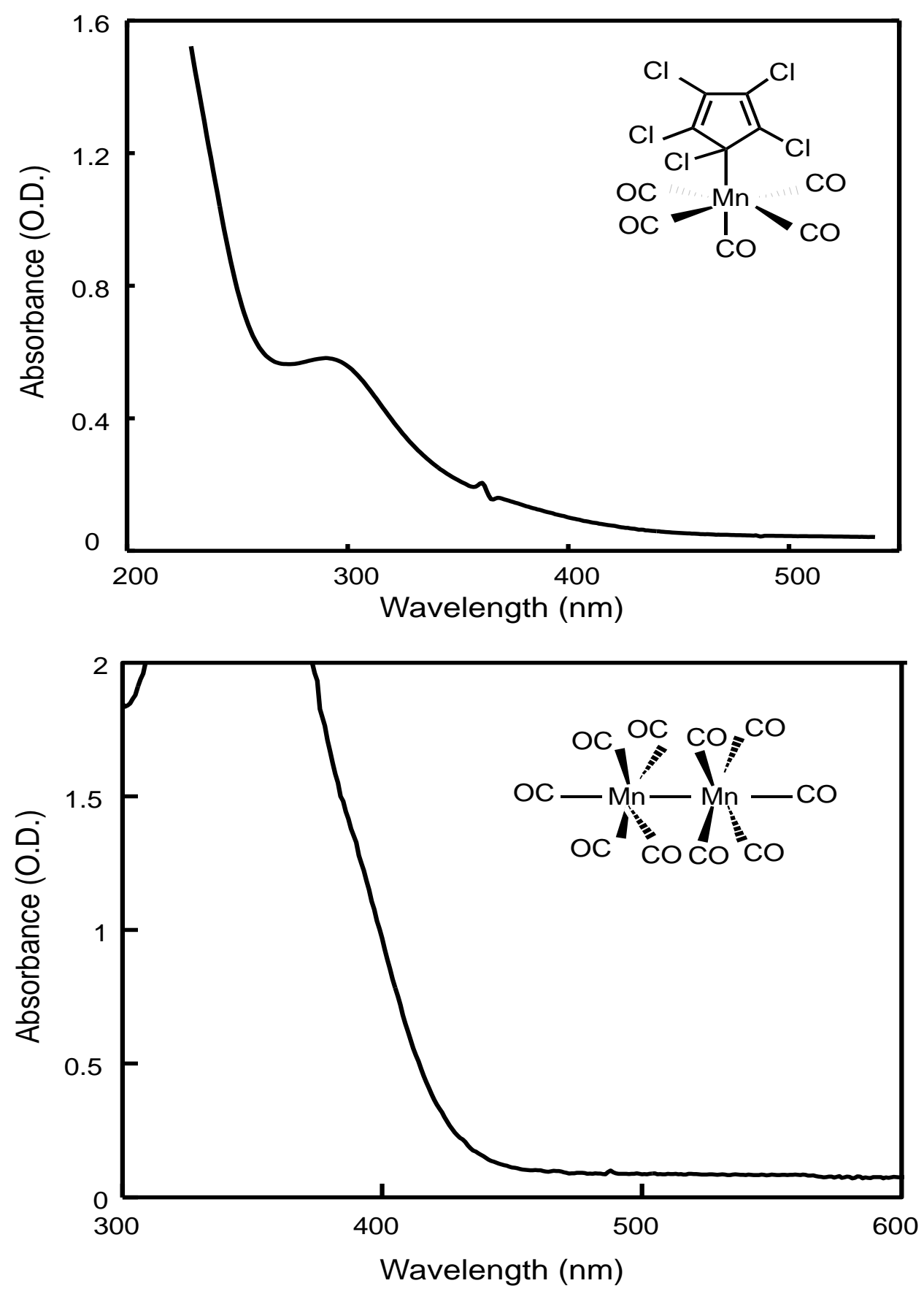

Figure 5.1: Ultraviolet-visible spectra of $\eta^{1}-\left(C_{5} C l_{5}\right) M n(C O)_{5}$ and $M n_{2}(C O)_{10}$. Electronic transitions responsible for the various peaks are discussed in the text. 
transitions between the manganese-manganese bond[80, 81].

The importance of these absorption features manifests itself in the choice of excitation wavelength used in experiments. By choosing a wavelength corresponding to particular transition it is possible to dissociate the appropriate ligand. The majority of the experiments discussed previously used UV light at $295 \mathrm{~nm}$ to initiate the dissociation of a carbonyl ligand. If a $400 \mathrm{~nm}$ pump beam is used, a LF transition will be excited leading to the breaking of a metal-metal bond. This allows, depending on the possibility of changing wavelengths, a great deal of selectivity in the questions to be addressed. For example, studies of metal-carbonyl clusters require the dissociation of a metal-metal bond whereas the alkyne rearrangement studies described in Chapter 3 required the loss of a carbonyl. Overall, initiating a reaction in this manner allows the study of reactions in a way that mimics the conditions that are of interest to synthetic chemists, such as photolytic catalysis. ${ }^{3}$

Using infrared light to probe the resulting dynamics takes advantage of the strong absorptions of metal-carbonyl species between $1700 \mathrm{~cm}^{-1}$ and $2100 \mathrm{~cm}^{-1}$, a region which is generally free from other absorptions. A vibrational absorption is only observed if a change in the electric dipole, $\mu$, occurs during the transition. While carbon monoxide, free carbonyl, has a very weak dipole moment, this increases considerably upon bonding to the metal center as an effect of the electron density of the metal. The strong infrared signal of

\footnotetext{
${ }^{3}$ There is some debate in the literature regarding the nature of the dissociative state, CT vs. LF, for the dissociation of a carbonyl. Regardless of the initially populated state, lower energy excitation will favor metal-metal bond dissociation while higher energy excitation will favor carbonyl dissociation[82, 83].
} 
the carbonyl stretching frequencies make them a valuable tool for understanding reaction dynamics.

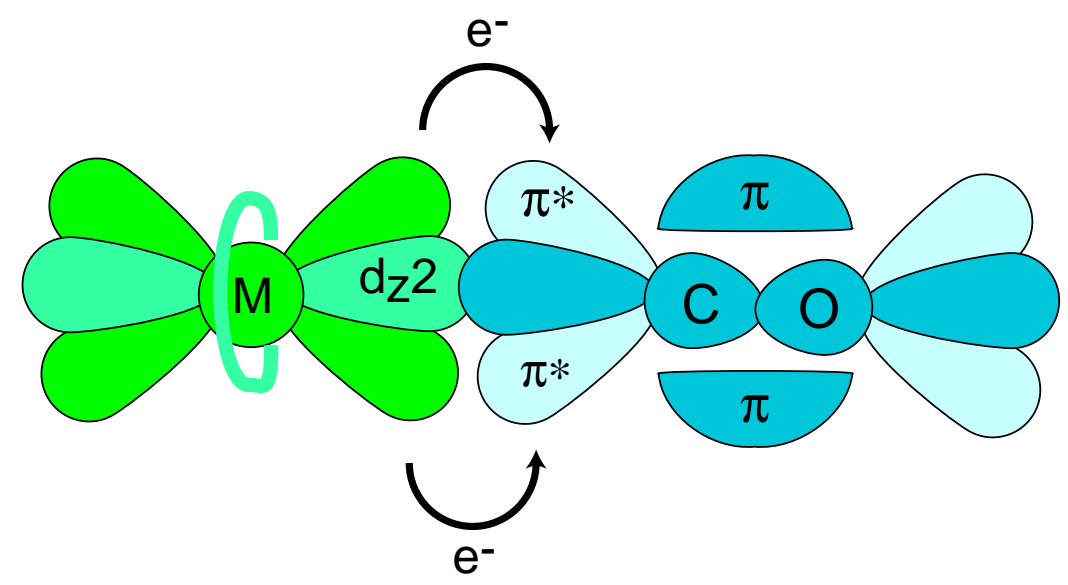

Figure 5.2: Back donation from metal to antibonding carbonyl orbitals.

Specifically, carbonyls are bonded to the metal through both $\sigma$-and $\pi$-bonds, as shown in Figure 5.2. Most important for infrared spectroscopy is the back-donation of electron density from the metal d-orbitals to the anti-bonding $\pi^{*}$ orbitals of the carbonyls. Due to this interaction with the anti-bonding orbitals of the carbonyl, any increase in electron density at the metal center, either through a change in ligand or neighboring solvent, is observed as a shift in carbonyl frequency to a lower energy (red-shift) as the carbonyl bond is weakened. Equation 5.1 shows the relationship between observed frequency and bond weakening which is written as the spring force constant, k. Essentially, added electron 
density to an anti-bonding orbital weakens the bond, lowering $\mathrm{k}$ by reducing the stiffness of the bond.

$$
\omega=\sqrt{\frac{k}{\mu}}
$$

Conversely, a loss of electron density on the metal center is observed as a shift to higher frequencies. By observing frequency shifts as well as changes in intensity it is possible to determine both the structure and relevant rates of intermediates and products.

Observing carbonyl vibrations in the infrared provides two pieces of information. The first is kinetic, as peaks increase or decrease as a function of time it is possible to determine rates for the formation and decay of intermediate species and/or products. The second piece of information is structural and it is this structural information which differentiates experiments probed in the infrared from those probed in the visible. Early work examining the mechanisms of organometallic reactions used probe wavelengths in the visible region of the spectrum $[16,53,84,85,86]$. Unfortunately, visible peaks are broad, as seen in UV-Vis spectra of Figure 5.1. This made it difficult to resolve separate intermediate species. In the infrared, peaks are narrower and the carbonyl groups are a good probe of local environments.

Following photoexcitation, a number of processes are initiated in nearly all solutionphase reactions. A photon of UV light imparts approximately $100 \mathrm{kcal} / \mathrm{mol}$ of energy to the solute molecule. In the case of a typical metal-carbonyl bond, $40 \mathrm{kcal} / \mathrm{mol}$ is needed 
to dissociate the carbonyl. The excess energy is dissipated to the surrounding solvent molecules in approximately $100 \mathrm{ps,} \mathrm{depending} \mathrm{on} \mathrm{the} \mathrm{coupling} \mathrm{of} \mathrm{the} \mathrm{solute-solvent} \mathrm{vibra-}$ tional modes. This fast dissipation of excess energy is generally believed to prevent the dissociation of subsequent carbonyls[53, 87, 88]. However, $\eta^{1}-\left(C_{5} C l_{5}\right) M n(C O)_{5}$, demonstrates two-carbonyl loss following photoexcitation suggesting that the exact nature of the excited state may play an important role in carbonyl loss[19]. The experimental signature of vibrational relaxation is a broad and red-shifted absorption. As the higher vibrational levels relax, absorptions will narrow and shift to the blue due to the anharmonic nature of the potential well $[14,55,56,57]$.

Geminate recombination, the solvent caging effect originally observed for the dissociation of small molecules[89], is also present following the photodissociation of an organometallic ligand. Using the example of carbonyl loss, geminate recombination will occur within approximately 150 fs as the surrounding solvent molecules form a cage from which the carbonyl cannot escape. It then rebinds to the metal center to form the parent $\operatorname{species}[14,90,91]$.

Following ligand dissociation, the metal center is coordinatively unsaturated, generally being left with sixteen instead of the preferred eighteen electrons. Often the electron density will be replaced by the formation of a weak, approximately $5 \mathrm{kcal} / \mathrm{mol}$, bond with a neighboring solvent molecule. This process occurs in less than $10 \mathrm{ps}$ depending on the bond strength of the organometallic-solvent complex $[15,74]$. In the case of alkyne poly- 
merization, and various other reaction mechanisms such as $\mathrm{C}-\mathrm{H}$ activation, solvation is the first step in the formation of a final product[70,69].

\subsection{Lasers}

There have been two laser systems used for the research described in this thesis. As the general principles of each laser system are the same, the following discussion will describe the important aspects of ultrafast spectroscopy and specific details will be provided in the following chapters. Laboratory-specific details such as alignment procedures will be included in the Appendix.

\subsubsection{Oscillator}

The central component in an ultrafast laser system is the oscillator which produces the ultrafast laser pulses. Current ultrafast lasers, like all lasers, consist of a gain medium, excited by an independent source, within a cavity. The key component of current ultrafast lasers is the titanium:sapphire gain medium which has a large bandwidth centered at 800 nm. This large bandwidth will allow for a number of different frequencies to oscillate within the cavity. Constructive interference between these modes will create a lens effect within the Ti:Sapphire crystal due to the optical Kerr Effect, specifically the dependence of the index of refraction, $\mathrm{n}$, on intensity (I). 


$$
n=n_{0}+n_{2} I,
$$

The nonlinear change in the index of refraction as a function of intensity allows the existence of a different mode shape and path for the constructively interfered modelocked beam as compared to the continuous wave beam. This difference in mode enables the cavity to be optimized for the propagation of the modelocked beam. In practice, the oscillator requires a noise spike to generate mode-locking, the cavity can then be optimized for the mode-locked beam allowing for the generation of a train of femtosecond pulses[92]. These oscillators, built from a Murnane and Kapteyn design, are capable of producing pulses of less than $100 \mathrm{fs}$ with repetition rates of $\mathrm{MHz}$ and output powers of approximately $1 \mathrm{~W}$ of tunable $800 \mathrm{~nm}$ light. They are typically pumped by argon-ion or diode lasers with frequencies around $532 \mathrm{~nm}$. The oscillators based on this design are fantastic and the systems are generally easy to construct.

\subsubsection{Amplifier}

Unfortunately, UV-pump IR-probe experiments require the use of ultraviolet and infrared light, not just $800 \mathrm{~nm}$ near-infrared. To generate additional frequencies it is necessary to use a series of non-linear crystals that require more power in order to generate a sufficient amount of power at the new frequency. For this reason, the ultrafast pulses generated by the oscillator are amplified, previously in this laboratory by a series of dye- 
amplifiers and currently with a regenerative amplifier. Both methods have been described in detail previously, the dye amplification by Asplund[93] and the regenerative amplification by Kotz[74] and Hunziker[94]. For that reason, only a brief overview will be provided.

The oscillators used in both laser systems are identical, but differences in amplification methods result in vastly different laser systems. The first system made use of a series of Bethune cells through which laser dye was flowed. The amplification method in this system is relatively intuitive. The dye is excited by a separate pump laser, in this case a Q-switched Nd:YAG, the pulse from the oscillator interacts with the excited dye resulting in a stimulated emission. Unfortunately the use of dyes leads to a number of difficulties in using the system and the nine dye cells required for this system created nine times the difficulties. Problems include dye contamination, poor mode and thermal effects. A solid-state amplifier operates with the same principles of external excitation and stimulated emission, but replaces the dye cells with a single titanium:sapphire crystal.

The amplification of ultrafast pulses results in intensities well above the damage threshold of the optics necessary for a solid state amplifier. For this reason, pulses must be stretched (lengthened in time), using a dispersive grating, prior to entry into the amplifier. After amplification, the lengthened pulses are compressed using a second, or possibly the same, diffraction grating. This method is referred to as chirped pulse amplification (CPA). Following dispersion on the grating the red edge of the beam will travel a shorter distance than the blue. This is described as "chirping" the pulse. Chirped pulse amplification, in the 
form of either a regenerative or multipass amplifier, provides a number of advantages over dye amplification. The end result is a more stable and more easily aligned laser system.

\subsection{Nonlinear Crystals}

Each laser system described in the following chapters required a different combination of nonlinear crystals to generate the necessary ultraviolet and infrared light. Commercial systems exist, but it is often quite simple to build a system to meet your specifications. The TOPAS, an optical parametric amplifier manufactured by Light Conversion, will also be discussed. While specific nonlinear methods will be described in the following chapters, it is perhaps worthwhile to discuss the general properties of nonlinear crystals.

Harris Group tradition has dictated that the properties of nonlinear crystals be learned soon after joining the group when given the job of finding the correct crystal to buy for the new and exciting project to be started. For that reason, the discussion of nonlinear crystals will be phrased in terms of practical applications. The four parameters that a crystal buyer are asked to specify are material, length, Type, and phase-matching angle. Considering how one might choose these parameters should provide a rough guide to the general principles involved in the use of nonlinear optics for frequency conversion. To begin, what are these parameters and why do they matter? In the end, a user would like to be able to convert the given frequency to the desired frequency with the maximum efficiency. 
With regards to frequency conversion, the first choice to consider is that of material. Specific crystal materials have been developed for specific applications. The choice of these materials is dictated by physical limitations, i.e. crystal $\mathrm{x}$ will absorb wavelength $\mathrm{y}$, as well as practical considerations such as durability, hardness, and ease of growth. For most conversion processes one will be interested in, there will be an established set of materials for such a process. The decision of which exact crystal to choose will depend on specific laboratory conditions. If, for whatever reason, your lab were wet, you would need to choose a crystal that is not hydroscopic (or build the proper enclosure). Other details are more important. One major consideration is damage threshold. Nonlinear crystals require high intensity laser beams. A material is only considered nonlinear if the electric field through the crystal, or liquid or gas, is strong enough to cause the oscillations of the the electrons to respond nonlinearly, i.e. in a spring model, the restoring force is no longer proportional to the displacement. Once the electrons oscillate at a frequency other than that of the initial frequency, a new wavelength of light is produced as determined by the conservation of energy. Crystals with higher damage thresholds will be less likely to burn, but may suffer other drawbacks.

The other parameters; length, Type, and phase-matching, may affect the choice of material, but have a larger role in efficiency. The conversion of one wavelength into another is not generally a highly efficient process and no one wants to waste valuable power. The power relationship for the generation of a second harmonic ("doubling") frequency is below; 


$$
P_{\text {doubled }} \alpha l^{2} \frac{P_{\text {fundamental }}^{2}}{A}\left(\frac{\sin ^{2} \Delta \phi}{(\Delta \phi)^{2}}\right) \text {, }
$$

At this point, $\mathrm{P}$, the input power, and $\mathrm{A}$, the area of the beam, are most likely wellestablished. By choosing the correct crystal parameters it is possible to optimize 1 , the length, and $\frac{\sin ^{2} \Delta \phi}{(\Delta \phi)^{2}}$, the phase matching conditions to maximize $\mathrm{P}_{\text {doubled }}$, the power of the converted frequency. The choice of length is a compromise, you cannot use an infinitely long crystal without suffering from increases in dispersion and absorption. An infinitely short crystal would not allow the distance, determined by wavelength, necessary for the frequency conversion to occur.

The phase-matching condition in the above equation can vary between 0 and 1. This value can be maximized by ensuring that there is no region of destructive interference within the crystal. As the new wavelength is produced it continues to propagate through the crystal along with the initial wavelength. However, the differences in wavelength will cause them to propagate at different speeds. It is then possible that additional frequency conversion will occur at a point that puts it directly out of phase from the initial conversion. The interaction of two oppositely phased wavelengths will cause destructive interference. The birefringence of the crystal will compensate for this by slowing one wavelength relative to the other, but it is unlikely that the crystal is birefringent in an exactly compensating manner. Birefringence can be tuned either by changing the temperature of the crystal or the angle. This angle should be chosen such that the velocity of the input wavelength along the 
ordinary axis is equal to the velocity of the converted wavelength along the extraordinary axis. In reality, it is difficult to tune the angle exactly due to walkoff effects. If light propagates at an angle oblique to the optic axis, light traveling along the e axis will walkoff from the o axis.

The Type of crystal refers to I or II and their defined polarization conventions. In Type I crystals the input wavelength is polarized along the ordinary axis, the output along the extraordinary. Within Type II crystals, the input wavelength is split evenly between the ordinary and extraordinary axes the output wavelength is along the extraordinary axis. While certain crystals may be more efficient for certain conversions with a specific Type, the addition of half-wave plates to obtain the necessary polarizations must also be weighed. Overall, it's important to chose a crystal that will maximize output without being destroyed $[95,96]$.

\subsection{Time Resolution}

Following the generation of UV and IR light, both beams are overlapped at the sample. The polarization of both UV and IR are set to the magic angle, $54.7^{\circ}$, to avoid polarization artifacts. The path of the UV includes a motor-controlled translation stage. By stepping the stage a time delay is created between the time the UV light excites the sample and the infrared pulse probes the sample. This time delay allows for the collection 
of kinetic information.

The time delay of the various laser systems described above is determined by the difference in time between the photoexcitation of the species, with the pump beam, and the arrival of the infrared probe pulse. The time difference is controlled by a computercontrolled motorized stage (Klinger, now owned by Newport) on which two of the pump line mirrors are aligned to work as a retroreflector. Changing the distance that the pump beam travels controls the time at which the laser pulse interacts with the sample. For example, at what is referred to as t0 (time zero) the pump and probe interact with the sample at the same time. If the stage position is changed such that the pump beam travels a shorter distance, it will now excite the sample before the probe. The time delay is related to the distance the stage is moved by considering the speed of light.

$$
\text { time delay }=\frac{\text { distance of stage from } t_{0}}{c},
$$

By collecting probe signals as the stage is stepped through various distances it is possible to compile of probe signal as a function of time, essentially kinetics.

The time resolution is minimally bound by the length of each stage step. The current stage has a step size of $1 \mu \mathrm{m}$, equivalent to $3.3 \mathrm{fs}$. The distance the light will travel is actually twice this distance as the light must travel both to and from the mirror resulting in a stage step of $6.6 \mathrm{fs}$. The maximum delay is determined by the length of the stage, in this case $15 \mathrm{~cm}$ or 150,000 steps, resulting in a maximum delay of $1 \mathrm{~ns}$. 


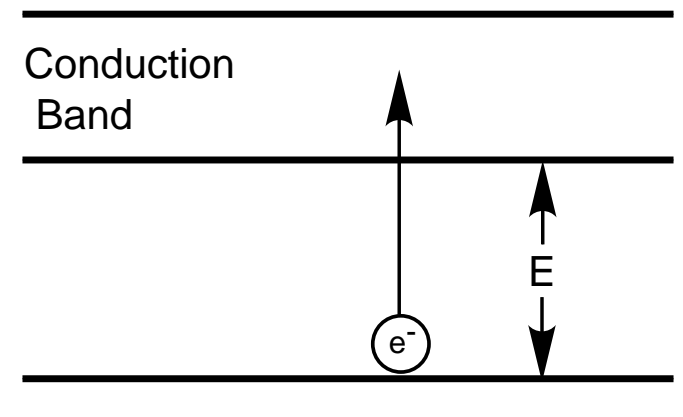

Valence Band

Figure 5.3: Basic concept of intrinsic photoconductors.

\subsection{Detection}

After exciting the sample, the UV light is dumped to a beam block. The IR is aligned into a monochromator or spectrograph which uses a diffraction grating to disperse the beam into individual wavelengths. Depending on the grating position, a different wavelength exits the monochromator or spectrograph and is detected by an infrared detector. The details of both will be described in their respective laser sections. The laser systems described in the following chapters have made use of two types of detectors, single-element and array mercury-cadmium-telluride (MCT) detectors. The physics of both are identical. $\mathrm{MCT}$ is an intrinsic photoconductor, as shown in Figure 5.3.

An incident photon with energy greater than E will excite an electron from the valence to conduction band and create an electron-hole pair leading to an increase in conductivity. MCT is generally sensitive over a range of 2-11 $\mu \mathrm{m}$ although individual detectors 
are optimized for a particular region. This, along with a 1 ns response time, makes MCT a good material for a variety of applications. The one disadvantage is the need for constant cooling to $77 \mathrm{~K}$ with liquid nitrogen. Due to the small bandgap, the thermal excitation of electrons would compete with the signal. An array detector is an extension of the singleelement detector composed of multiple MCT elements. The electronics of an array are more complex, but the overall idea is the same $[97,98]$.

The photons detected are converted to voltages which are sampled, digitized, and recorded as described in individual laser sections. Data analysis is accomplished using Matlab [99] scripts.

\subsection{Data Analysis}

Data analysis was performed using various Matlab programs written by Payne. Analysis will differ depending on the quality of the data, but a general description is provided below. Peaks and bleaches can be fit with a Gaussian or Voight function. The change in peak (or Gaussian) height as a function of time is then used to obtain kinetic information. Error analysis can be carried out with standard techniques such as a calculation of standard deviation or the Monte Carlo bootstrap method. The kinetic data was fit using standard exponential rate equations. 


\subsection{Electronic Structure Calculations}

Traditional electronic structure calculations are generally extensions of the variational Hartree-Fock (HF) methods learned in undergraduate quantum mechanics. In this method the wavefunction is described as a product of orbitals. The parameters of these orbitals are then varied to determine the lowest energy wavefunction. Density functional theory (DFT), developed by Kohn and Hohenberg in 1964, recognizes that the entire wavefunction contains more information than necessary. The actual value desired is the ground state energy, $\mathrm{E}_{0}$ which can be described as a functional of the electron density, $\rho$.

$$
E_{0}=E_{0}\left[\rho_{0}\right]
$$

In 1965 Kohn and Sham developed a method for calculating $\rho$ using Kohn-Sham orbitals, $\theta$ which resemble true orbitals, but have no real physical significance, instead they only allow for the calculation of the electron density.

$$
\rho=\sum_{i=1}^{n}\left|\theta_{i}\right|^{2}
$$

The ground state energy, $\mathrm{E}_{0}$, is then calculable although the functional is only approximate. While the exact functional is unknown, much research is aimed at developing improved functionals, the quality of which is based on comparison to either experimental or high-level electronic structure calculations[100]. 
For DFT methods the important parameters for the casual user of commercial packages such as Gaussian98[75] and Jaguar[41], are the functional and basis set. While the exact functional is unknown, many good approximations exist and one is chosen based on the system under study. Most calculations in this thesis used Becke's 3-parameter Lee Yang Parr functional, B3LYP[44].

$$
\begin{array}{r}
E_{x c}=\left(1-a_{0}-a_{x}\right) E_{x}^{\prime}+a_{0} E_{x}^{\prime \prime}+a_{x} E_{x}^{\prime \prime \prime}+\left(1-a_{c}\right) E_{c}^{\prime}+a_{c} E_{c}^{\prime \prime} \\
a_{0}=0.20, a_{x}=0.72, a_{c}=0.81
\end{array}
$$

It is a hybrid functional, using gradient corrected exchange, $\mathrm{E}_{x}$, and correlation, $\mathrm{E}_{c}$ energy terms. ${ }^{4}$ Basic DFT uses a uniform electron gas model, gradient corrections account for variations in electron density as a function of position. The parameters, $\mathrm{a}_{0}$, $\mathrm{a}_{x}$, and $\mathrm{a}_{c}$ were calculated to fit experimental values for molecular atomization energies. This functional performed well in benchmark studies in which calculated geometries were compared to crystal structures of similar organometallic complexes[45, 46].

The basis set, for both HF and DFT calculations, determines the representation of the orbitals. Slater-type-orbitals (STOs) are approximated by various combinations of Gaussian functions to reduce the size of the calculation. To further reduce the size of the calculation, organometallic systems often make use of Effective Core Potentials (ECPs). The ECP replaces the two-electron Coulomb and exchange operators that describe the

\footnotetext{
${ }^{4}$ The prime notation represents the unique energy terms with which we will not be concerned here.
} 
interaction between the valence and core electrons with a one-electron operator calculated from experimental or high-level electronic structure calculations. A common basis set used in this thesis is $\mathrm{LACVP}^{* *}[47,48,49]$. An ECP is used for the metal center and the other atom are represented with the $6-31 G^{* *}$ basis set. This is shorthand for the representation of the inner shell electrons with 6 primitive gaussian functions and the valence electrons with 3 primitive guassians and 1 contracted gaussian. The contracted gaussian gaussian, $\chi$,

$$
\chi=\sum_{u} d g_{u}
$$

is a linear combination of primitive gaussians, $\mathrm{g}_{u}$, that increases the speed of the calculation by reducing the number of variational coefficients. This basis set is a double-zeta basis set meaning that each Slater Type Orbital (STO),

$$
S T O=\frac{\left(2 \zeta / a_{0}\right)^{n+1 / 2}}{[(2 n) !]^{1 / 2}} r^{n-1} e^{\zeta r / a_{0}} Y_{l}^{m}(\theta, \phi),
$$

is replaced with two STOs with differing orbital exponents, $\zeta$. The ${ }^{* *}$ notation indicates that polarization functions have been include for both the main group elements $\left(^{*}\right)$ and hydrogen and helium $\left({ }^{* *}\right)$. The polarization functions compensate for the shift and distortion of the center of charge of an atomic orbital upon the formation of a molecule. This is accomplished by the inclusion of STOs with 1 values greater than the 1 those of the ground state atoms. The other appendage often seen is ++ for the inclusion of diffuse functions for the main group 
$\left.{ }^{*}\right)$ and hydrogen $(* *)$. These functions use a small orbital exponent to represent electron density at distances far from the nucleus, i.e. anions and hydrogen-bonded dimers[100].

The use of electronic structures has proven invaluable in the determination of short-lived intermediates. Frequencies can be calculated from geometries and compared to experimental results. Various frequency scaling methods have been proposed although none have been used for these calculations, as discussed in the text.

\subsection{Conclusions}

The study of organometallic reactions in solution requires ultrafast infrared spectroscopy. In combination with electronic structure calculations, it is possible to determine the structure and lifetime of each intermediate formed during the course of a reaction. The specific laser systems used are discussed in the following chapters. 


\section{Chapter 6}

\section{Laser I: Ultrafast}

\section{Ultraviolet-Pump Infrared-Probe}

\section{Solid State Laser System}

While the laser described below was never used for regular data collection, a description of its construction provides a good overview of contemporary solid state laser systems. The theses of Kenneth Kotz[74] and Lukas Hunziker[94] outline the construction of the oscillator, stretcher, regenerative amplifier, and compressor and will not be repeated here. It is important to note that this design is not unique and is nearly identical to designs used by many commercial laser companies and other ultrafast laboratories. Following the 
generation of amplified ultrafast laser pulses at $800 \mathrm{~nm}$, the choice of design depends on the experiment. In this case, it is necessary to generate ultraviolet, visible, and infrared light to both initiate and observe the reaction dynamics. The use of various nonlinear techniques for the generation of ultraviolet and infrared light is described below. A diagram of the entire laser system is shown in Figure 6.1.

\subsection{Generation of Ultraviolet, Visible, and Infrared Light}

The use of ultraviolet and visible light is a common method for the initiation of a photochemical reaction. In the case of organometallic complexes the proper choice of wavelength can be used to dissociate specific bonds as discussed in Chapter 5. Infrared radiation provides a means of monitoring both the kinetics and structural changes of the resulting reaction mechanism. The output of the femtosecond oscillator discussed in the thesis of Kenneth Kotz is centered at $806 \mathrm{~nm}[74]$. This wavelength is fairly standard for titanium:sapphire oscillators. The generation of additional frequencies is accomplished with the use of nonlinear optical techniques. A brief description of nonlinear optics has been provided previously and is applicable to the generation of the entire range of frequencies discussed below.

The femtosecond pulses generated by the oscillator are subsequently stretched, amplified, and then compressed. The output of the compressor provides sufficient power 


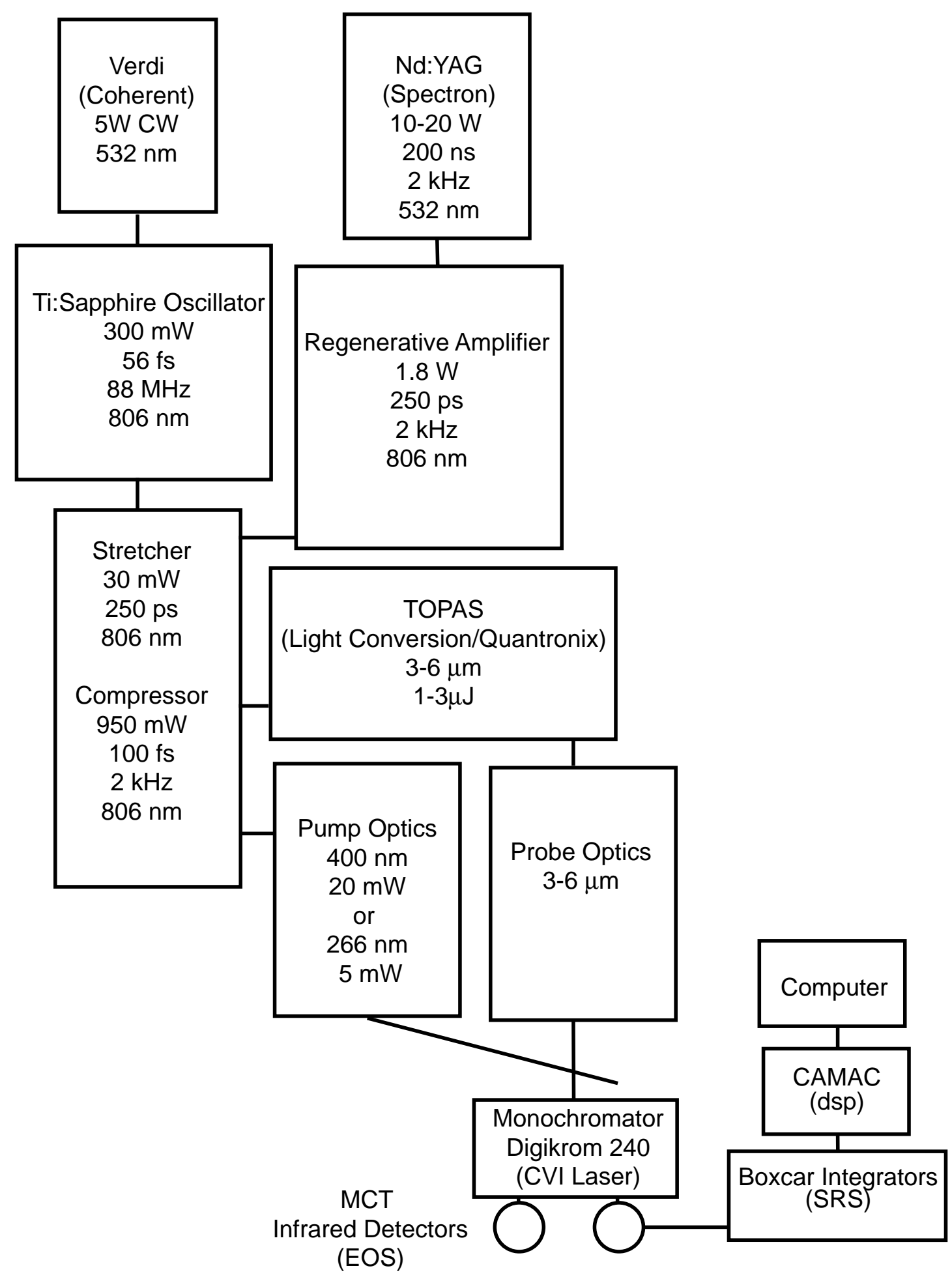

Figure 6.1: Schematic of solid state laser system. 
for the conversion of the $800 \mathrm{~nm}$ light into more useful frequencies. The output of the compressor is immediately split with a beamsplitter sending $80 \%$ of the light to the TOPAS for conversion to infrared. The remaining $20 \%$ is reflected out of the compressor for converion to ultraviolet or visible light depending on the experiment. The following discussion is divided into two sections, describing the generation of the pump (ultraviolet or visible) and probe (infrared) beams.

\subsubsection{Pump-Ultraviolet and Visible}

Figure 6.2 shows the configuration of the ultraviolet or visible pump beam. A brief description of the optics is in Table 6.1.1. Following the beamsplitter, the $800 \mathrm{~nm}$ light travels a strangely indirect path to a motorized translation stage. Both the strangely indirect path and the translation stage play a role in the time delay between the pump and probe beam resulting in the time resolution of the experiment. In order for the pump and probe beam to be temporally overlapped at the sample, they must travel the same distance. As the probe line has more configurational contraints, the length of the pump line is adjusted to match the length of the probe line. In this case that involves some extra travel between two mirrors. The distances between the pump and probe lines is set to be equal when the retroreflector on the translation stage is near the far end. This allows for longer time resolution as the stage is stepped forward. Creating a shorter pump line creates a longer delay before the probe interacts with the sample. 


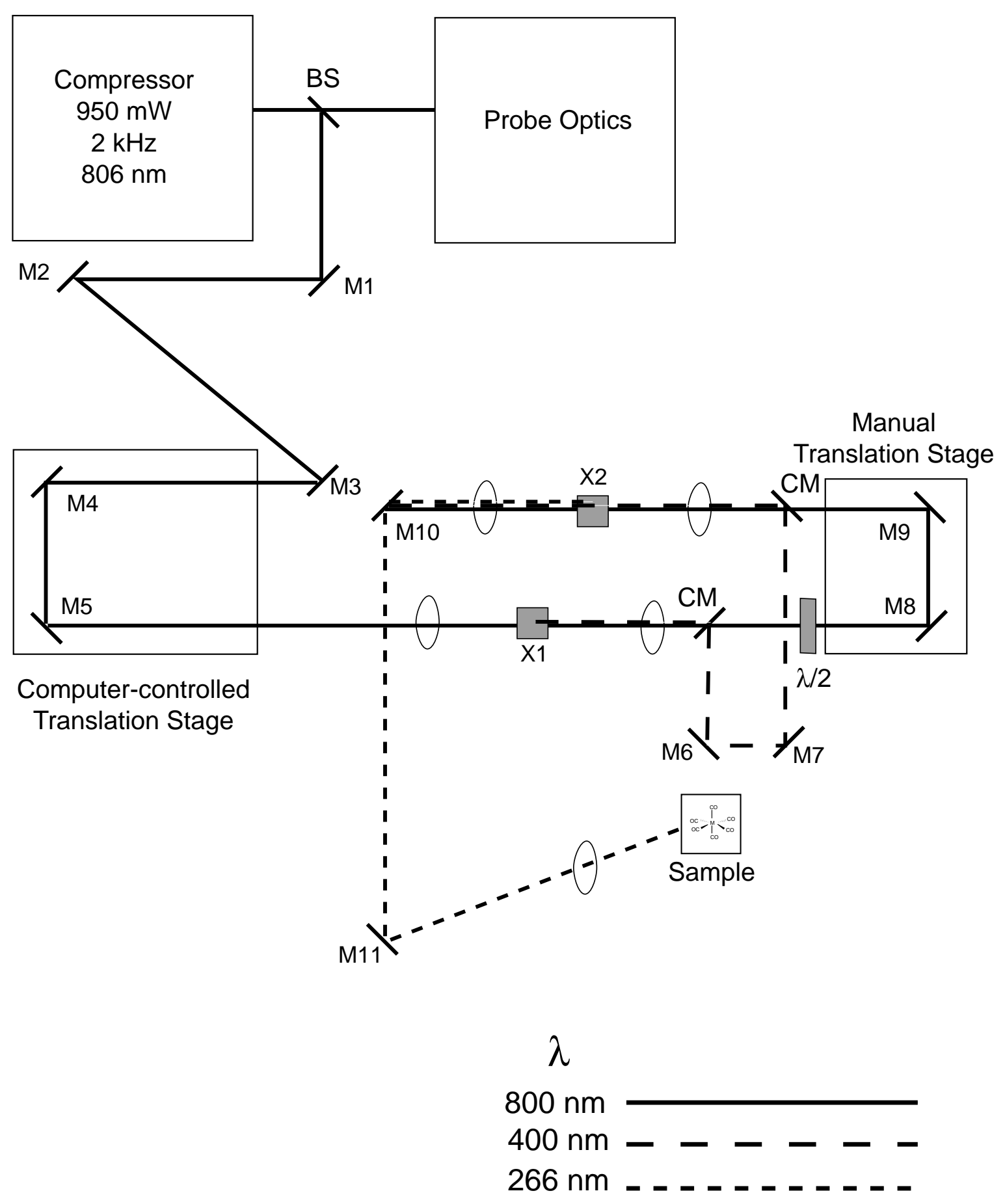

Figure 6.2: Schematic of ultraviolet/visible pump beam. 
Table 6.1: Description of Optics Used for the Generation of Ultraviolet Light

\begin{tabular}{cc}
\hline \hline & Optic \\
\cline { 2 - 2 } BS & Beam splitter $80 / 20$ at $800 \mathrm{~nm}$ \\
$\mathrm{X} 1$ & BBO Crystal $0.5 \mathrm{~mm} 28.96$ Type I (Photox) \\
$\mathrm{X} 2$ & BBO Crystal $1 \mathrm{~mm} 43.80$ Type I (Photox) \\
$\lambda / 2$ & $800 \mathrm{~nm}$ half-waveplate \\
CM & Pass $800 \mathrm{~nm}$, Reflect $400 \mathrm{~nm}$ (Newport) \\
M1-4, M8-9 & Broadband IR (CVI) \\
M6-7 & 400 nm optimized (Quantum Optics) \\
M10-11 & 266 nm optimized (Newport) \\
Lenses & CaF $_{2}$ (various vendors) \\
\hline \hline
\end{tabular}

From the translation stage the beam is focused into a Type I, $0.5 \mathrm{~mm}$ thick, $\beta$ barium borate $(\mathrm{BBO})$ crystal generating $20 \mathrm{~mW}$ of $400 \mathrm{~nm}$ light. After being recollimated the $400 \mathrm{~nm}$ light is reflect via a cold mirror to a set of retroreflectors while the unconverted $800 \mathrm{~nm}$ light is passed through a half-wave plate to a separate set of retroreflectors mounted on a manual translation stage. The half-wave plate rotates the polarization of the $800 \mathrm{~nm}$ light by 90 degrees. Changing the polarization of the $800 \mathrm{~nm}$ light makes it possible to use a higher efficiency Type I crystal for the generation of $266 \mathrm{~nm}$ light. The alternative method is to remove the half-wave plate and use a Type II crystal, but even though the removal of the half-wave plate does reduce the overall loss of the system the higher efficiency of the Type I crystal results in a greater power output. The use of a manual translation stage allows the temporal overlap of the $400 \mathrm{~nm}$ and $800 \mathrm{~nm}$ light to be controlled as they pass 
through the second BBO crystal.

Following retroreflection of both beams the $800 \mathrm{~nm}$ light is passed through the cold mirror from which the $400 \mathrm{~nm}$ is reflected. The two beams can now be spatially and temporally overlapped as they pass through a Type I, $1 \mathrm{~mm}$ thick, BBO crystal. Sumfrequency generation (SFG) results in the production of light at $266 \mathrm{~nm}$ with $1 \mu \mathrm{J}$ pulse energy. Following recollimation, the $266 \mathrm{~nm}$ light is aligned onto the sample and overlapped with the infrared. Experiments with $400 \mathrm{~nm}$ light are carried out by removing the second BBO crystal, blocking the $800 \mathrm{~nm}$ light, and replacing the final turning mirrors with mirrors optimized for $400 \mathrm{~nm}$.

\subsubsection{Probe-Infrared}

\section{TOPAS and DFG}

The TOPAS (Traveling-wave Optical Parametric Amplification by Superfluorescence) and accompanying DFG (difference frequency generation) attachment are both products of Light Conversion, Lithuania, and are distributed in the U.S. by Quantronix. The TOPAS is essentially a computer-controlled optical parametric amplifier with a few special features. In general, OPAs use the same principles as nonlinear optics to generate two new frequencies of light.

$$
\omega_{1}=\omega_{2}+\omega_{3}
$$


Varying $\omega_{1}$ allows tunability of $\omega_{2}$ and $\omega_{3}$ which are referred to as the signal and idler. ${ }^{1}$ The tunability depends on the phase-matching conditions of the system. The luxury of the TOPAS is that these conditions, mainly the crystal angle, are on motorized mounts controlled by a computer. Upon entering the TOPAS the beam is split, in the end making five passes through a BBO crystal. The first pass produces the broadband superfluorescence which serves as $\omega_{1}$. This is different from other OPA designs which traditionally use white light as the broadband source. The second pass shapes the beam, the third provides a small amount of amplification, the fourth is considered an additional pre-amplification step, and the fifth provides the large amplification. The output, following proper filtering, are two near-infrared beams, the frequency of which are chosen by the user.

Difference frequency generation produces mid-infrared light by taking the difference between the near-IR signal and idler. The two near-IR beams travel through an index of refraction matching material that results in temporal overlap at a $\mathrm{AgGaS}_{2}$ crystal. The angle of the crystal is tuned with a motorized mount to guarantee proper phase matching.

\section{Infrared Path: Signal and Reference}

Figure 6.3 shows the optical path following generation of the infrared light. A brief description of the components is given in Table 6.1.2.

Following output from the TOPAS, IR light is focused onto a beamsplitter. Roughly

\footnotetext{
${ }^{1}$ Convention dictates that the shorter wavelength be called "signal" and the longer "idler".
} 
6.1. GENERATION OF ULTRAVIOLET, VISIBLE, AND INFRARED LIGHT

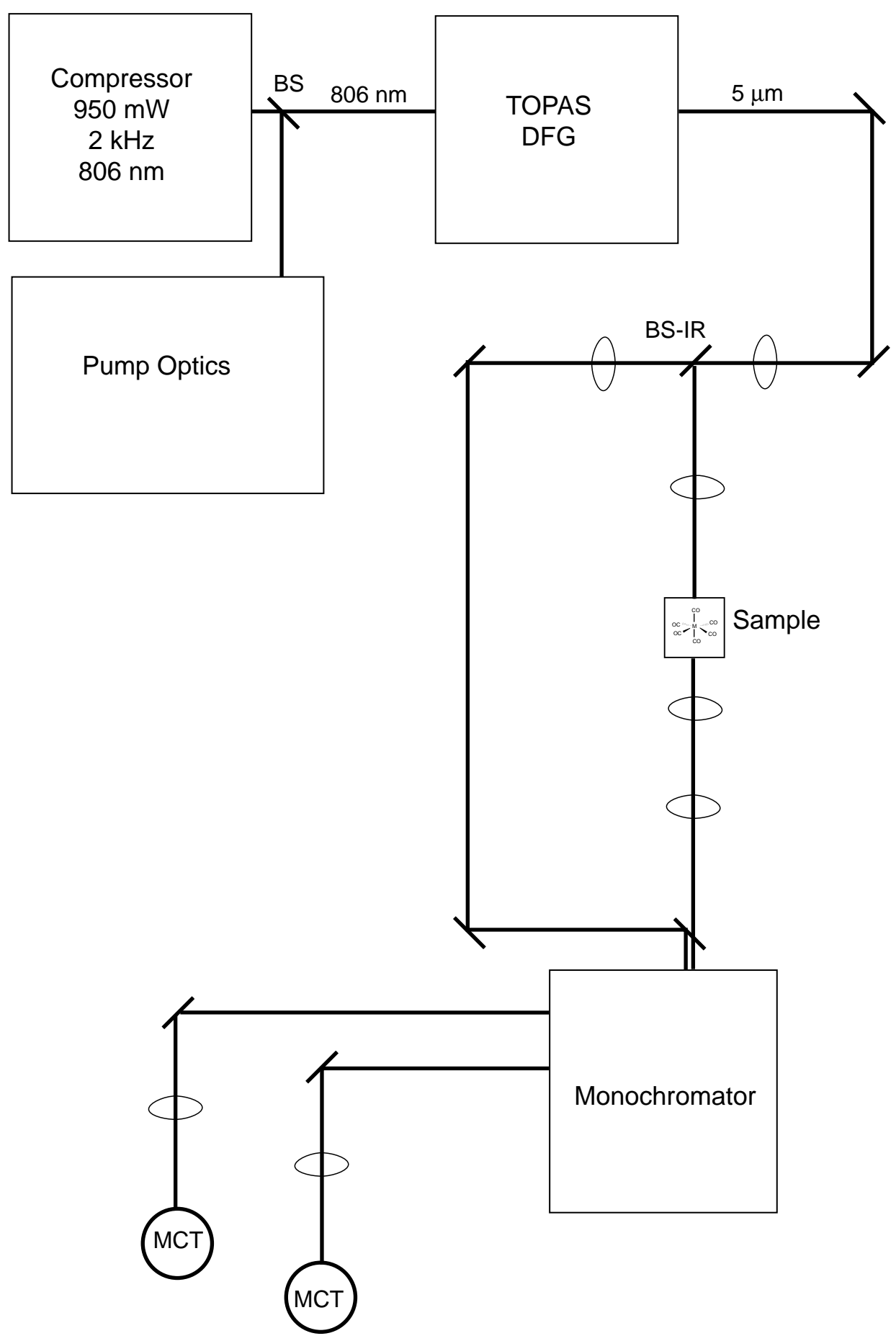

Figure 6.3: Schematic of infrared probe beam. 
Table 6.2: Description of Infrared Probe Line Components

\begin{tabular}{cc}
\hline \hline & Component \\
\cline { 2 - 2 } BS & Beam splitter $80 / 20$ at $800 \mathrm{~nm}$ \\
TOPAS/DFG & Tunable mid-IR OPA (Light Conversion) \\
BS-IR & Beam splitter $50 / 50$ at $5 \mu \mathrm{m}$ \\
Monochromator & Digikrom 240, 4000 nm blaze (CVI) \\
MCT & Mercury-Cadmium-Telluride Infrard Detectors (EOS) \\
Mirrors & Broadband IR (CVI) \\
Lenses & $\mathrm{CaF}_{2}$ (various vendors) \\
& \\
\hline \hline
\end{tabular}

half the light passes through the beam splitter to form the reference line while the rest is reflected to form the signal line. The use of a signal and reference line to calculate a normalized signal (signal/reference) allows for the cancellation of systematic noise in the laser, long-term power drifts, and atmospheric absorptions. The signal line is focused into the sample and overlapped with the pump beam. The signal and reference are both aligned into the monochromator. A slight offset in beam height allows the signal to pass over the final turning mirror o the reference. The monochromator (Digikrom 240) is a standard Czerny-Turner design in which both beams are reflected from a curved mirror onto a grating. The grating angle is controlled by an external computer for the selection of frequency. The light is then reflected from the grating to a second curved mirror which reflects the light out of the monochromator. With the proper choice of slit spacing and beam focus $4 \mathrm{~cm}^{-1}$ resolution is obtained. Upon exiting the monochromator the heights are reversed 
such that the reference passed above the signal. Both beams are focused into single element mercury-cadmium-telluride (MCT) detectors cooled to liquid nitrogen temperature.

\subsection{Overlap of Pump and Probe}

Proper overlap of the pump and probe beams is essential for observing actual reaction dynamics. Clearly exciting one molecule and observing a separate molecule would not yield any information regarding the reaction mechanism. Ideally, the probe beam is few hundred microns on the sample with the pump beam slightly larger to guarantee only excited molecules are observed. Spatial overlap is accomplished by positioning a pinhole at the exact place where the sample will be placed in the path of the probe beam. The pump beam is then adjusted to be centered at the same pinhole. Temporal overlap is accomplished when the pump and probe pulses interact at the sample at the same time, referred to at t0. It is at this point that the UV and IR have traveled the same distance from the initial beamsplitter. As discussed previously, this position is controlled by fixing the length of the pump line, preferably with the pump mirrors near the back of the translation stage, to equal the length of the probe line. A thin silicon, or other appropriate material such as GaAs, wafer can be used to check the temporal and spatial overlap. While silicon will filter a fraction of the IR, the excitation of an electron by the pump beam, with either ultraviolet or visible light, will create a much stronger filter. At t0, and until approximately $1 \mathrm{~ns}$ 
afterwards, a drop in IR signal following photoexcitation by the pump beam will guarantee that the pump and probe beams are overlapped. Before t0, ie. with the stage moved further back, the pump beam will have no affect on the IR signal as a result of the probe beam interacting with the sample before the pump. The t0 signal, observed as a fast decrease in IR intensity, can also be used to cross-correlate the pump and probe thereby giving the length of the pulses.

\subsection{Detector, Electronics, and Software}

Two matched mercury-cadmium-telluride (MCT) detectors (EOS-the worst company ever) were used to detected the signal and reference photons. The output from the detectors was sampled with a two boxcar integrator (SRS). The boxcar integrators both reduced noise, as is their general purpose, and provided the necessary impedance matching for the analog-to-digital converter. The noise reduction by boxcar integrators takes advantage of a reduction of bandwidth to reduce the amount of white noise present. In the case of a low-duty cycle in which the signal is only present for a short time compared to the time between signal events, measuring a signal during spaced intervals reduces the amount of white noise sampled.

The integrated signal and reference are digitized by an eleven bit CAMAC (Com-

puter Automated Measurement And Control). Bits are read to a Pentium II processer via 
a GPIB card. Software, which served to both query the CAMAC and calculate and change in absorption, was written in C. 


\section{Chapter 7}

\section{Laser II: Ultrafast}

\section{Ultraviolet-Pump Infrared-Probe Dye-Amplified Laser System}

\section{$7.1 \quad$ Overview}

The work described in this thesis has been carried out using a number of different experimental systems. The bulk of experimental measurements were taken using an ultrafast dye-amplified laser system built by Mr. Haw Yang and Dr. Tim Lian (now Professors Yang and Lian). This laser system was based on a standard titanium:sapphire oscillator the 
pulses of which were amplified by a series of Bethune dye cells. Its construction is described by Matthew Asplund[93] and Tim Lian[14]. This system was plagued by noise, typically $30 \%$, and a slow repititon rate of $30 \mathrm{~Hz}$. The use of a 256X256 MCT array detector to collect $70 \mathrm{~cm}^{-1}$ spectra at each laser shot alleviated some of the pain associated with such a slow rate of data collection, but still resulted in week-long data collection sessions and noisy data. The specifications relevant to this thesis are described below.

\subsection{Experimental Details}

The output of a Ti:sapphire oscillator was amplified in series of dye amplifiers pumped by a $30-\mathrm{Hz} \mathrm{Nd}$ YAG laser at $532 \mathrm{~nm}$. The dye amplifiers were used to generate three beams with wavelengths of $800 \mathrm{~nm}, 690 \mathrm{~nm}$, and $580 \mathrm{~nm}$ or $650 \mathrm{~nm}$. Difference frequency generation in a lithium iodate crystal was used to generate mid-infrared light centered at $5 \mu \mathrm{m}$. Ultraviolet light was generated by doubling either $580 \mathrm{~nm}$ or $650 \mathrm{~nm}$ light in a potassium diphosphate crystal to generate either $290 \mathrm{~nm}$ or $325 \mathrm{~nm}$ light. The specific wavelength of UV light was determined by the choice of dye in the amplification

system. The resulting $295 \mathrm{~nm}$ or $325 \mathrm{~nm}$ UV photons, with energy of $6 \mu \mathrm{J} /$ pulse, were focused into a disk of $200 \mu \mathrm{m}$ diameter at the sample to initiate chemical reactions. The $1 \mu \mathrm{J}$ IR pulses were centered at $5 \mu \mathrm{m}$ with a temporal full-width-half-maximum of about $70 \mathrm{fs}$ and a spectral band width of about $200 \mathrm{~cm}^{-1}$. 
Shot-to-shot fluctuation of the IR probe pulses were minimized by normalizing the signal beam against a reference beam. These two beams were then focused into an astigmatism-corrected spectrographic monochromator (SpectraPro-150, Acton Research Corp., $150 \mathrm{gv} / \mathrm{mm}, 4.0 \mu \mathrm{m}$ blazed) to form two spectrally resolved images on a focal-planearray (FPA) IR detector. The two frequency-resolved images were digitized by two windows of $12 \times 200$ pixels, which allowed simultaneous normalization of a $70 \mathrm{~cm}^{-1}$ spectrum. The censoring chip of the detector was an engineer grade, 256x256-element $\mathrm{HgCdTe}$ (MCT) matrix of dimensions $1.28 \times 1.28 \mathrm{~cm}^{2}$ (or $50 \mathrm{x} 50 \mu \mathrm{m}^{2}$ per pixel). During the course of an experiment, the censoring chip and its immediate circuits were kept in contact with a 4-L liquid nitrogen dewar to increase its sensitivity in the IR range. The typical spectral and temporal resolution for this setup were $4 \mathrm{~cm}^{-1}$ and $300 \mathrm{fs}$, respectively. The polarizations of the pump and the probe pulse were set at the magic angle $\left(54.7^{\circ}\right)$ to ensure that all signals were due to population dynamics. 


\section{Chapter 8}

\section{Step-Scan FTIR: Observation of}

\section{Dynamics on the Nano- to}

\section{Microsecond Timescale}

\subsection{Overview}

For many reactions the initial step or steps occur on a timescale of femto- to picoseconds. However, the final product may or may not be formed on these timescales depending on the reaction being studied. In the ring slip work discussed in Chapter 2, the chlorine-bridged species that is an intermediate in the formation of the ring slipped product 
is formed in $50 \mathrm{ps}$. The ring slipped product, is formed in $130 \mathrm{~ns}[19]$. This is just one example in which nanosecond timescales play an important role in connecting the ultrafast formation of intermediates to final products. ${ }^{1}$

On occasions when a full understanding of the reaction mechanism cannot be determined on the ultrafast timescale it is necessary to either use a different stage with a longer length or use a different method. While it is easy to imagine experiments with a different stage, it is not necessarily a trivial adjustment to make for various experimental reasons. Fortunately, the Harris Group has had a long-standing collaboration with Dr. Heinz Frei of the Physical Biosciences Division of Lawrence Berkeley National Laboratory, located just meters from our laboratory in the neighboring Calvin Laboratory. Dr. Frei specializes in the study of zeolites and catalysis using a step-scan and rapid-scan FTIR.

Step-scan FTIR, or similarly rapid-scan FTIR, allows for the collection of data on the nano- to millisecond timescale. Dr. Frei's system is well-described in three sources, Frei's early paper describing the step-scan FTIR[40], a manual from Bruker[101], and the thesis of Matthew Asplund[93]. For that reason, only a brief description is provided here. On a practical level the step-scan experiment, including the pump laser, provides a perfect compliment to the ultrafast laser system. Both systems pump in the UV or visible and probe in the IR. For the step-scan, the pump laser is a separate component that is used as an excitation source and electronics trigger. Specifically, it is a GCR Nd:YAG laser from

\footnotetext{
${ }^{1}$ The best example of this can be seen in the carbon-hydrogen bond activation work[69, 70].
} 
which any harmonic can be chosen as an excitation source, i.e. $1064 \mathrm{~nm}, 532 \mathrm{~nm}, 355 \mathrm{~nm}$ or $266 \mathrm{~nm}$. Typically $355 \mathrm{~nm}$ or $266 \mathrm{~nm}$ are used to provide similar excitation wavelengths to those used in the ultrafast experiments. The pump beam is aligned from one room, through a small wall division and focused onto the sample using a set of optics which can be placed in the step-scan. These optics are described in Asplund's thesis and are stored in Frei's lab. The probe beam consists of broadband IR produced by a Globar ceramic source, exactly as in a conventional FTIR.

The main question raised by the preceding paragraph may be the mention of a "small wall division" and to address this the essential mechanism of a step-scan FTIR must be discussed. In many ways step-scan and rapid-scan are quite similar, however, the details described below will refer only to step-scan. Step-scan provides information on the nanosecond to microsecond timescales and it is often this region which is of interest as it picks up where ultrafast experiments leave off. In many ways, a step-scan FTIR is similar to a conventional FTIR with the exception that the movable mirror of the Michelson interferometer is STEPPED through each position. At each mirror position the sample is excited and an interferogram is collected at various time delays. With this method, a series of interferograms with differing time delays are obtained. A Fourier transform is performed and the interferograms are converted to spectra. In this manner, the time resolution of the experiment is only limited by the response time of the detector and electronics. The stepping of the mirror is very sensitive to vibration. For this reason, the pump laser is in 
the room next door, the step-scan is on a vibrationally isolated (floating) table, and there is no talking or air-conditioning during experiments.

As in the ultrafast experiments, the sample is flowed to provide fresh sample for each excitation pulse. The optical set-up used contains a holder for a Harrick cell at the point where the pump and probe beams overlap. However, the sample cannot be flowed using a peristaltic pump directly as the vibration from the pump would affect the stepping mirror. For this reason, a gravity flow system, sketched in Payne Notebook 1, is used to isolate the pump vibration from the sample.

\subsection{Detection}

During the course of an experiment there are few parameters adjustable by the user. The main parameters include; the number of CoAdds, referring to averages at each mirror position, folding limits, resolution, pump wavelength, as discussed above, and detector. The choice of detector is generally between two typical infrared detectors mercury cadmium telluride (MCT) or indium antinomide (InSb). The two materials have different windows of sensitivity and response times. The choice should be based on the experiment in question. Resolution and folding limits are also dependent on the experiment. Increasing both will increase the time needed to collect data and may be, especially in the case of folding limits, unnecessarily large if not chosen well. Resolution is typically set to $4 \mathrm{~cm}^{-1}$. 
Folding limits are equivalent to the high and low frequency bounds. In order to sample data correctly, they must be chosen such that no light is absorbed beyond them. To limit this range, it is best to chose a filter that is transparent over the frequencies being examined, ie. $1800-2200 \mathrm{~cm}^{-1}$, but absorbs $100 \%$ of the infrared outside of the limits. Dr. Frei has a number of filters from which to choose. They are placed in front of the detector and a spectrum is recorded. The folding limits are then set as the frequencies at which all light is absorbed.

Throughout the collection of data transient (AC) and static (DC) scans are alternated. This provides a check of the condition of the sample and are then used to calculate the absorption using Equation 8.1. This equation includes an amplification factor, $\beta$, that corrects for the amplification of AC signal and attenuation of DC signal as set by the electronics.

$$
A=-\log \frac{A C+(\beta x D C)}{\beta x D C}
$$

This calculation can be done using a macro, written by Kenneth Kotz and described in the thesis of Matthew Asplund[93], for the step-scan software. The macro (Master) is easily edited, requiring only an output path and folding limits to be updated. The macro will request a pair of $\mathrm{AC}$ and $\mathrm{DC}$ scans and perform an absorption calculation with an output of time slices. There are some software difficulties with handling this data due to incompatible operating systems. Currently, February, 2003, the best method is to run 
the macro on the step-scan computer and then $\mathrm{ftp}$ the converted data to the Windows NT computer in Frei's laboratory. A CD can be recorded from this computer and easily transported. This data can be loaded into Matlab using the Loader and Rewriter programs written by Kotz and then treated as necessary.

\subsection{Experimental Details}

The spectra recorded on the nanosecond to microsecond timescale were recorded using a Step-Scan FTIR spectrometer described elsewhere. The instrument was based on a Bruker IFS-88 FTIR with a special scanner module to allow step-scanning. An MCT detector with 37 ns temporal full-width-half-maximum (FWHM) measured from the IR scatter of 1064-nm light from a YAG laser was used. The IR light was focused in the cavity with two 10-mm focal length $\mathrm{BaF}_{2}$ lenses, which gave beam sizes smaller than comparable curved mirrors, allowing increased IR throughput and less sample degradation. The sample was photo-excited with 10-ns pulses from the quadrupled YAG. 


\section{Chapter 9}

\section{Conclusions}

Ultrafast ultraviolet-pump infrared-probe spectroscopy has been shown to be an important method for the determination of organometallic reaction mechanisms. Of particular interest are reactions which require the rearrangement of a complex ligand. Two reaction mechanisms involving ligand rearrangement include ring slip and alkyne polymerization. The mechanism of ring slip has been determined using a combination of spectroscopy and density functional theory calculations. It has been found that the reaction proceeds through a previously unknown intermediate and forms a seventeen-electron complex not predicted by traditional molecular orbital theory. Alkyne polymerization via a hexacarbonyl catalyst has been shown to proceed differently than predicted, most likely requiring multiphoton initiation. The single photon dissociation of a carbonyl results in the formation of a longlived alkyne-solvated intermediate species rather than the vinylidenemetal precursor for 
polymerization.

In terms of future work there are numerous suggestions to be made. The Langevin calculations described in Chapter 4 are incomplete. Ideally, a comparison would be made between alcohol and alkyne simulations. Depending on the outcome, it may be necessary to reformulate the friction term, possibly using parameters drawn from dielectric relaxation models.

While the work described in Chapters 2 and 3 is fairly complete, the methods used could easily be extended to other organometallic systems in which ligand rearrangement plays an essential role. Two examples include $\beta$-hydrogen elimination and cluster reactivity. Additionally, countless other reaction mechanisms could be more fully understood with the use of ultrafast infrared spectroscopy. 


\section{Bibliography}

[1] K. Ziegler, (1963), 1953 provides a rough start date for the many papers for which the Nobel Prized was awarded.

[2] G. Natta, (1963), 1953 provides a rough start date for the many papers for which the Nobel Prized was awarded.

[3] E. O. Fischer, (1973), 1952 provides a rough start date for the many papers for which the Nobel Prized was awarded.

[4] G. Wilkinson, J. Organomet. Chem. 100, 273 (1975), numerous citations exist. The cited reference is a personal recollection of the work.

[5] G. W. Parshall and S. D. Ittel, Homogeneous Catalysis, 2 ed. (Wiley, New York, 1992).

[6] G. L. Geoffroy and M. S. Wrighton, Organometallic Photochemistry (Academic Press, New York, 1979).

[7] A. H. Janowicz and R. G. Bergman, J. Am. Chem. Soc. 104, 352 (1982). 
[8] J. K. Hoyano and W. A. G. Graham, J. Am. Chem. Soc. 104, 3723 (1982).

[9] J. A. Labinger and J. E. Bercaw, Nature 417, 507 (2002).

[10] Organometallic Chemistry of the Transition Metals, 2 ed. (Wiley, New York, 1994).

[11] B. A. Arndtsen, R. G. Bergman, T. A. Mobley, and T. H. Peterson, Acc. Chem. Res. 28, 154 (1995), generally all R.G. Bergman work.

[12] W. D. Jones, Acc. Chem. Res. 36, 140 (2003), see also additional W.D. Jones work.

[13] T. P. Dougherty and E. J. Heilweil, J. Chem. Phys. 100, 4006 (1994).

[14] T. Q. Lian, S. E. Bromberg, M. C. Asplund, H. Yang, and C. B. Harris, J. Phys. Chem. 100, 11994 (1996).

[15] K. T. Kotz, H. Yang, P. T. Snee, C. K. Payne, and C. B. Harris, J. Organomet. Chem. 596, $183(2000)$.

[16] J. D. Simon and X. L. Xie, J. Phys. Chem. 91, 5538 (1987).

[17] T. J. Burkey, J. Am. Chem. Soc. 112, 8329 (1990).

[18] S. L. Zhang, G. R. Dobson, and T. L. Brown, J. Am. Chem. Soc. 113, 6908 (1991).

[19] C. K. Payne, P. T. Snee, K. T. Kotz, H. Yang, L. L. Schafer, T. D. Tilley, and C. B. Harris, J. Am. Chem. Soc. 123, 7425 (2001). 
[20] C. Bruneau and P. H. Dixneuf, Acc. Chem. Res. 32, 311 (1999).

[21] Y. Wakatsuki, N. Koga, H. Yamazaki, and K. Morokuma, J. Am. Chem. Soc. 116, 8105 (1994).

[22] I. de los Rios, M. J. Tenorio, M. C. Puerta, and P. Valerga, J. Am. Chem. Soc. 119, 6529 (1997).

[23] C. K. Payne, P. T. Snee, E. Garrard, J. Shanoski, and J. V. Zanten, in preparation (2003).

[24] F. Basolo, New J. Chem. 18, 19 (1994).

[25] J. M. O'Connor and C. P. Casey, Chem. Rev. 87, 307 (1987).

[26] P. Vest, J. Anhaus, H. C. Bajaj, and R. V. Eldik, Organometallics 10, 818 (1991).

[27] C. P. Casey, J. M. O'Connor, and K. J. Haller, J. Am. Chem. Soc. 107, 1241 (1985).

[28] M. E. Rerek and F. Basolo, J. Am. Chem. Soc. 106, 5908 (1984).

[29] A. J. Rest, I. Whitwell, and W. A. G. Graham, J. Chem. Soc., Chem. Commun. 624 (1984).

[30] D. P. Drolet and A. J. Lees, J. Am. Chem. Soc. 114, 4186 (1992).

[31] M. Ephritikhine and M. L. H. Green, J. Chem. Soc., Chem. Commun. 926 (1976). 
[32] G. K. Yang and R. G. Bergman, Organometallics 4, 129 (1985).

[33] H. G. Schuster-Woldan and F. Basolo, J. Am. Chem. Soc. 88, 1657 (1966).

[34] H. G. Alt and E. Samuel, Chem. Soc. Rev. 27, 323 (1998).

[35] S. K. Chowdhury, U. Samanta, V. G. Puranik, and A. Sarkar, Organometallics 16, 2618 (1997)

[36] G. Huttner, H. H. Brintzinger, L. G. Bell, P. Friedrich, V. Bejenke, and D. Neugebauer, J. Organomet. Chem. 145, 329 (1978).

[37] K. M. Young and M. S. Wrighton, J. Am. Chem. Soc. 112, 157 (1990).

[38] K. J. Reimer and A. Shaver, Inorg. Chem. 14, 2707 (1975).

[39] K. J. Reimer and A. Shaver, J. Organomet. Chem. 93, 239 (1975).

[40] H. Sun and H. Frei, J. Phys. Chem. B. 101, 205 (1997).

[41] Jaguar, 3.5 ed., Schrodinger, Inc., Portland, OR, 1998.

[42] A. D. Becke, J. Chem. Phys. 98, 5648 (1993).

[43] C. T. Lee, W. T. Yang, and R. G. Parr, Phys. Rev. B 37, 785 (1988).

[44] P. J. Stephens, F. J. Devlin, C. F. Chabalowski, and M. J. Frisch, J. Phys. Chem. 98, 11623 (1994). 
[45] A. Ricca and C. W. Bauschlicher, Theor. Chim. Acta 92, 123 (1995).

[46] M. N. Glukhovtsev, R. D. Bach, and C. J. Nagel, J. Phys. Chem. A 101, 316 (1997).

[47] M. M. Francl, W. J. Petro, W. J. Hehre, J. S. Binkley, M. S. Gordon, D. J. DeFrees, and J. A. Pople, J. Chem. Phys. 77, 3654 (1982).

[48] W. J. Hehre, R. Ditchfield, and J. A. Pople, J. Chem. Phys. 56, 2257 (1972).

[49] P. J. Hay and W. R. Wadt, J. Chem. Phys. 82, 299 (1985).

[50] D. J. Tannor, B. Marten, R. Murphy, R. A. Friesner, D. Sitkoff, A. Nicholls, M. Ringnalda, W. A. G. III, and B. Honig, J. Am. Chem. Soc. 116, 11875 (1994).

[51] B. Marten, K. Kim, C. Cortis, R. A. Friesner, R. B. Murphy, M. N. Ringnalda, D. Sitkoff, and B. Honig, J. Phys. Chem. 100, 11775 (1996).

[52] L. F. Veiros, J. Organomet. Chem. 587, 221 (1999).

[53] J. D. Simon and K. S. Peters, Chem. Phys. Lett. 98, 53 (1983).

[54] P. T. Snee, C. K. Payne, S. D. Mebane, K. T. Kotz, and C. B. Harris, J. Am. Chem. Soc. 123, 6909 (2001).

[55] M. W. George, T. P. Dougherty, and E. J. Heilweil, J. Phys. Chem. 100, 201 (1996).

[56] T. P. Dougherty and E. J. Heilweil, Chem. Phys. Lett. 227, 19 (1994). 
[57] T. P. Dougherty, W. T. Grubbs, and E. J. Heilweil, J. Phys. Chem. 98, 9396 (1994).

[58] S. P. Church, H. Hermann, F. W. Grevels, and K. Schaffner, J. Chem. Soc., Chem. Commun. 785 (1984).

[59] R. B. Hitam, K. A. Mahmoud, and A. J. Rest, J. Organomet. Chem. 291, 321 (1985).

[60] H. L. Clarke and N. J. Fitzpatrick, J. Organomet. Chem. 40, 379 (1972).

[61] G. Davidson and D. C. Andrews, J. Chem. Soc., Dalton Trans. 126 (1972).

[62] B. Gita and G. Sundararajan, Tet. Lett. 34, 6123 (1993).

[63] J. A. K. DuPlessis and H. C. M. Vosloo, J. Mol. Cat. 65, 51 (1991).

[64] S. J. Landon, P. M. Shulman, and G. L. Geoffroy, J. Am. Chem. Soc. 107, 6739 (1985).

[65] T. Szymanska-Buzar, A. J. Downs, T. M. Greene, and A. S. Marshall, J. Omet. Chem. 495, 149 (1995).

[66] T. Szymanska-Buzar, A. J. Downs, T. M. Greene, and A. S. Marshall, J. Omet. Chem. 495, 163 (1995).

[67] T. Szymanska-Buzar and K. Kern, J. Omet. Chem. 622, 74 (2001).

[68] P. O. Stoutland and R. G. Bergman, J. Am. Chem. Soc. 107, 4581 (1985). 
[69] S. E. Bromberg, H. Yang, M. C. Asplund, T. Lian, B. K. McNamara, K. T. Kotz, J. S. Yeston, M. Wilkens, H. Frei, R. G. Bergman, and C. B. Harris, Science 278, 260 (1997).

[70] M. C. Asplund, P. T. Snee, J. S. Yeston, M. J. Wilkens, C. K. Payne, H. Yang, K. T. Kotz, H. Frei, R. G. Bergman, and C. B. Harris, J. Am. Chem. Soc. 124, 10605 (2002).

[71] H. Yang, M. C. Asplund, K. T. Kotz, M. J. Wilkens, H. Frei, and C. B. Harris, J. Am. Chem. Soc. 120, 10154 (1998).

[72] J. P. Hansen and I. R. McDonald, Theory of Simple Liquids, 2 ed. (Academic Press Inc., London, 1986).

[73] P. T. Snee, C. K. Payne, K. T. Kotz, H. Yang, and C. B. Harris, J. Am. Chem. Soc. 123, 2255 (2001).

[74] K. T. Kotz, Ph.D. thesis, University of California, Berkeley, 2001.

[75] Gaussian98, Gaussian, Inc., Carnegie, PA, 1998.

[76] CRC Handbook of Chemistry, 74 ed., edited by D. R. Lide (CRC Press, Boca Raton, FL, 1994).

[77] D. S. Viswanath and G. Natarajan, Data Book on the Viscosity of Liquids (Hemisphere Publishing Corporation, New York, 1989). 
[78] S. K. Garg and C. P. Smyth, J. Phys. Chem. 69, 1294 (1965).

[79] T. Sato and R. Buchner, J. Chem. Phys. 118, 4606 (2003).

[80] R. A. Levenson and H. B. Gray, J. Am. Chem. Soc. 97, 6042 (1975).

[81] M. S. Wrighton and D. S. Ginley, J. Am. Chem. Soc. 97, 2065 (1975).

[82] C. Pollak, A. Rosa, and E. J. Baerends, J. Am. Chem. Soc. 119, 7324 (1997).

[83] E. J. Baerends and A. Rosa, Coord. Chem. Rev. 177, 97 (1998).

[84] X. L. Xie and J. D. Simon, J. Am. Chem. Soc. 112, 1130 (1990).

[85] X. Xie and J. D. Simon, J. Phys. Chem. 93, 291 (1989).

[86] N. J. Tro, J. C. King, and C. B. Harris, Inorg. Chim. Acta 229, 469 (1995).

[87] L. J. Rothberg, N. J. Cooper, K. S. Peters, and V. Vaida, J. Am. Chem. Soc. 104, $3536(1982)$.

[88] J. A. Welch, K. S. Peters, and V. Vaida, J. Phys. Chem. 86, 1941 (1982).

[89] B. J. Schwartz, J. C. King, J. Z. Zhang, and C. B. Harris, Chem. Phys. Lett. 203, $503(1993)$.

[90] H. Yang, P. T. Snee, K. T. Kotz, C. K. Payne, and C. B. Harris, J. Am. Chem. Soc. 123, 4204 (2001). 
[91] J. Nasielski and A. Colas, Inorg. Chem. 17, 237 (1978).

[92] M. M. Murnane, H. C. Kapteyn, C. P. Huang, M. T. Asaki, and D. Garvey, ModeLocked Ti:Sapphire Laser. Designs and Guidelines for Constructing a Mode-Locked Ti:Sapphire Laser., 1.6 ed., Pullman, WA, 1992.

[93] M. C. Asplund, Ph.D. thesis, University of California, Berkeley, 1998.

[94] L. E. Hunziker, Ph.D. thesis, University of California, Berkeley, 1997.

[95] C. B. Hitz, Understanding Laser Technology, 2 ed. (PennWell, Tulsa, Oklahoma, 1991).

[96] W. Ubachs, in Nonlinear Optics, Department of Physics and Astronomy (Laser Center Vrije Universiteit, Amsterdam, 2001).

[97] Building Scientific Apparatus, 2 ed. (Addison-Wesley, New York, 1989).

[98] A. Rogalski, Infrared Physics and Technology 43, 187 (2002).

[99] Matlab, 6 ed., The MathWorks, Inc., 2001.

[100] I. N. Levine, Quantum Chemistry, 5 ed. (Prentice Hall, New Jersey, 2000).

[101] T. J. Johnson and G. Zachman, Introduction to Step-Scan FTIR.

[102] TOPAS User's Manual, Light Conversion, Ltd., Vilnius, Lithuania. 


\section{Chapter 10}

\section{Appendix: Alignment,}

\section{Maintenance, Comments}

Alignment procedures for much of the laser system described in Chapter 6 are detailed in the thesis of Kotz[74]. The exception to this is the TOPAS for which the alignment is described below. Additionally, the current alignment procedure for the oscillator is described as it has been realigned following Kotz's thesis. 


\subsection{Alignment and Maintenance of the Ti:Sapphire Oscil- lator}

The oscillator is described in Lukas Hunziker's[94] and Kenneth Kotz's[74] theses as well as the original construction notes from Kapteyn and Murnane[92]. It works perfectly. There should be no difficulty in obtaining mode-locked pulses of good power after a bit of optics cleaning and mirror tweaking. With the hopes that someone will use it, I will describe my alignment method below.

1-Use some spectroscopic quality methanol to clean the optics every few weeks.

2-Turn on the Verdi, open shutter, allow twenty minutes to warm-up.

3-Check for lasing. If the oscillator is not lasing use the procedure outlined in Kotz's thesis or the Murnane/Kapteyn manual.

4-Check for mode-locking (ML) by sliding P2 (notation from Kotz thesis, p. 93) through it's full translation. Use the camera detecting light from the diffraction grating to look for a slight blurring of the mode. (The CW mode is round, ML will appear as a horizontal streak on the screen.) If a slight blurring is observed, you are very close. Move P2 to the position where the blurring starts and tweak the high reflector (HR) to obtain a higher order mode, as opposed to $\mathrm{TEM}_{00}$. Iterate between tweaking end mirrors and adjusting P2.

5-If it is still not possible to ML, record the position of M2. Move it back (away from Ti:Sapph crystal) $1 \mathrm{~mm}$. Do this carefully. Optimize the position of the end mirrors to 
maximize the $\mathrm{CW}$ power. It should be possible to obtain at least $500 \mathrm{~mW}$. Move M2 to original position. This will lower the $\mathrm{CW}$ power, but should make it possible to $\mathrm{ML}$ the laser by slightly changing the cavity from that of an ideal CW cavity to that of a ML cavity. 6-Optimize. The laser will ML more easily from the higher-order modes, which are easily observed on the camera screen.

\subsection{Alignment of TOPAS}

The TOPAS is described in both the accompanying manual[102] and Chapter 6 of this thesis. The best source of assistance is Light Conversion, the Lithuanian company that designed the TOPAS. Both Donatas Podenas and Gediminas Veitas can be emailed with questions. Additionally, the Saykally group here at Berkeley is always helpful in answering questions. Software for the TOPAS is currently backed up and is also running on the monochromator computer in D-93. The TOPAS is in good working order with a few caveats.

Most importantly, the TOPAS is optimized specifically for the output of the com-

pressor. The slightest change in wavelength, beam, or power will not only change the calibration curve, i.e. entering $5000 \mathrm{~nm}$ will instead shift the wavelength to $5050 \mathrm{~nm}$, but will most likely make the TOPAS inoperable. The best option would be to realign and recalibrate using the method described in the TOPAS manual. If this is accomplished, or if 
you get very lucky, alignment of the TOPAS requires the following steps using the notation of Figure 10.1 kindly provided by Light Conversion.

1-Use two turning mirrors before the TOPAS to align the beam through A1 and A2. The fifth pass will be split off at BS1 and the fourth at BS2. At this point, you should be able to detect the production of superfluorescence after the nonlinear crystal. It will look bright green. White light at this point indicates the generation of supercontinuum resulting from too much power. See the TOPAS manual for instructions. Besides seeing bright green light, the fourth and fifth passes should be centered on M3 and M9, respectively.

2-Optimize the first three passes with the turning mirrors by removing the cover from the grating, DG, and checking to see that the green light is centered as it hits the grating.

3-Optimize the computer-controlled parameters such as crystal angle and delay time to maximize the green light.

4-Insert a card between M11 and M8 to check the overlap of the seed and pump beam. Optimize cautiously with M10 for the pump beam and M7 for the seed beam. The beams should be centered and overlapped without losing superfluorescence.

5-M11 and M8 share a mount which often slips from its ball bearings. This seems to be a common problem in the TOPAS. It will be obvious if it has happened since the mount will not be adjustable. To fix it, gently lift up the mount from the ball bearings and reset it. 6-If things are aligned well, the signal and idler should now be aligned on the DFG crystal, not shown in Figure 10.1. If not, use M8 to adjust. 


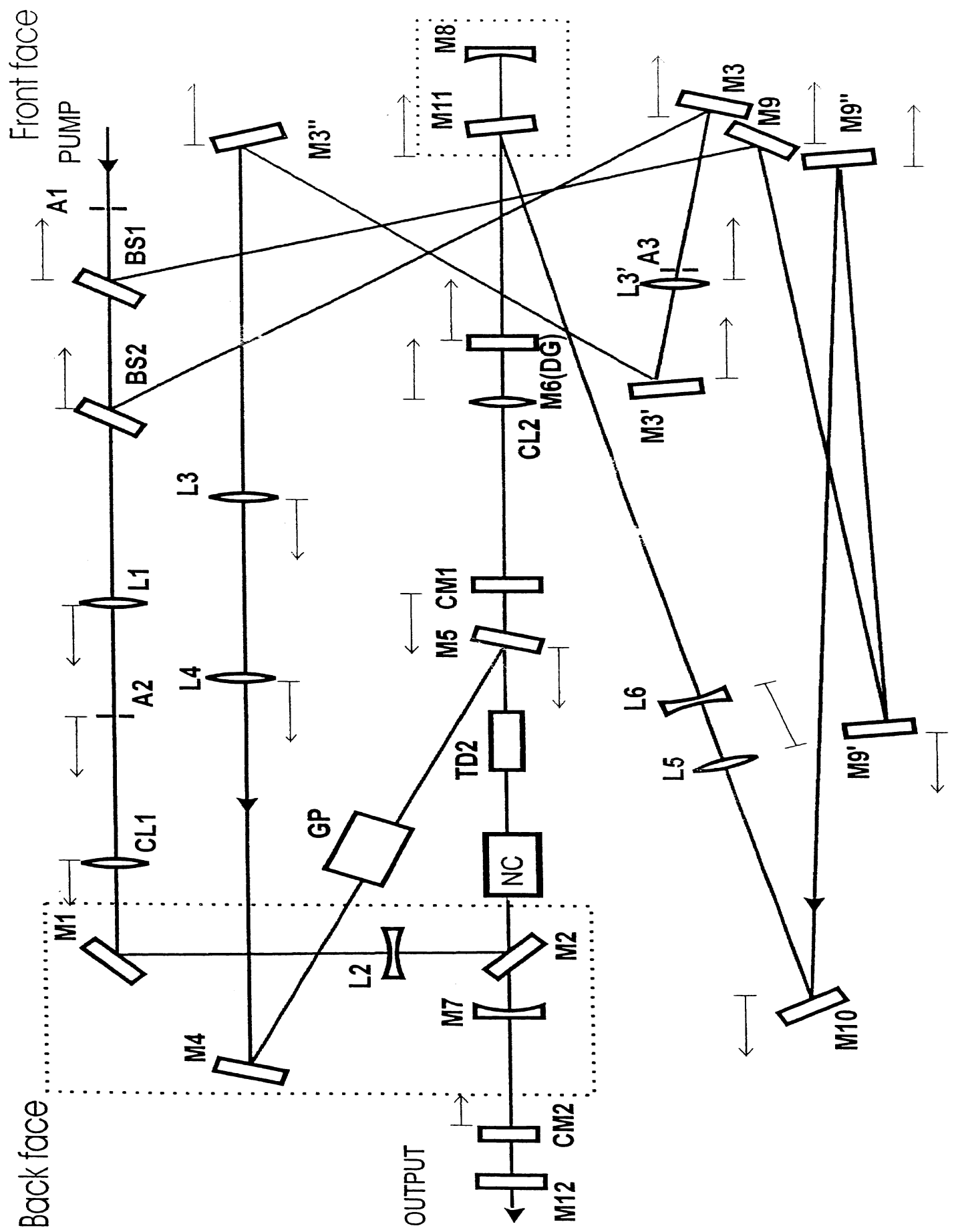

Figure 10.1: Optical diagram of TOPAS, provided by Light Conversion. 
7-Optimize A3 for power.

8-Use the computer to optimized DFG angle for power. Recheck other computer controlled parameters.

\subsection{Maintenance of Additional Laser Components}

While it would be lunacy to attempt to use the laser in D-91 for its intended purpose, there are some components worth maintaining. Described below are the various components in terms of their use and maintenance.

\subsubsection{Verdi Pump Laser for Oscillator}

This is in perfect working order although it will need a diode replacement in 2-4 years depending on use. Clean out the chiller and tubing before use.

\subsubsection{Nd:YAG Laser for Pumping the Regenerative Amplifier}

The Nd:YAG pump laser is not one of the components worth saving. General instability and temperature fluctuations make this laser unusable. If for some reason you were to use it, flashlamps are on the top shelf of the cabinet. Instructions for changing the flashlamp can be found in the manual and Notebook 2. Also, the filter and water should be replaced before any attempt to use it. 


\subsubsection{Regenerative Amplifier}

The amplifier is best described in the theses of Hunziker and Kotz. With a new pump laser to replace the Nd:YAG it may be salvageable with a great deal of realignment.

\subsubsection{Stretcher/Compressor}

This is best described in Kotz's thesis. A replacement grating is in the optics cabinet.

\subsubsection{Translation Stage}

The translation stage is from Klinger which has since been bought by Newport. See them for service or replacement.

\subsubsection{Monochromator}

The monochromator, Digikrom 240, suffers from two occasional problems. The first is known as the "Echo time-out" error which generally results from trying to enter information as the monochromator is processing information. This will cause it to dissociate from computer control and can be corrected by unplugging the monochromator and restarting the computer. This will take you through a set up file where you will be prompted to reenter the computer parameters-Digikrom 240, RS232, Com2. Parameters such as wavelength and slit width will need to be reset. 
Ironically, the second problem often occurs when the monochromator is unplugged and results in an incorrect resetting of the gratings. The monochromator can switch between two gratings, one for the visible and one for the infrared. If the monochromator confuses the two it will be obvious by the lack of IR passed through or by opening the monochromator cover and seeing which grating is being used for a particular wavelength. This requires resetting the factory settings. From the directory c:cvilaser, run 8-0114-D.exe. This program will ask you for the factory setting, read them from disk, file 10515c.nov. After restarting the computer setting the monochromator wavelength to $5000 \mathrm{~nm}$ should move Grating 2, Blazed at $4000 \mathrm{~nm}$, into position. If this does not work, try again, and again. If you need to edit $10515 \mathrm{c} . n$ nov to change the gratings be sure to save a copy.

\subsubsection{CAMAC/Boxcar Integrators/Computer}

The computer and programs for taking data are older than anyone reading this thesis and should not be used unnecessarily. The CAMAC and boxcar integrators are described in accompanying manuals and Notebook 2. They work well, but have been phased out by National Instruments. 